LPT-Orsay-05-65

\title{
Integrability of two-loop dilatation operator in gauge theories
}

\author{
A.V. Belitsky ${ }^{a}$, G.P. KorchemskY ${ }^{b}$, D. MÜLleR ${ }^{a}$ \\ ${ }^{a}$ Department of Physics and Astronomy, Arizona State University \\ Tempe, AZ 85287-1504, USA \\ ${ }^{b}$ Laboratoire de Physique Théorique ${ }^{1}$, Université de Paris XI \\ 91405 Orsay Cédex, France
}

\begin{abstract}
We study the two-loop dilatation operator in the noncompact $S L(2)$ sector of QCD and supersymmetric Yang-Mills theories with $\mathcal{N}=1,2,4$ supercharges. The analysis is performed for Wilson operators built from three quark/gaugino fields of the same helicity belonging to the fundamental/adjoint representation of the $S U(3) / S U\left(N_{c}\right)$ gauge group and involving an arbitrary number of covariant derivatives projected onto the light-cone. To one-loop order, the dilatation operator inherits the conformal symmetry of the classical theory and is given in the multi-color limit by a local Hamiltonian of the Heisenberg magnet with the spin operators being generators of the collinear subgroup of full (super)conformal group. Starting from two loops, the dilatation operator depends on the representation of the gauge group and, in addition, receives corrections stemming from the violation of the conformal symmetry. We compute its eigenspectrum and demonstrate that to two-loop order integrability survives the conformal symmetry breaking in the aforementioned gauge theories, but it is violated in QCD by the contribution of nonplanar diagrams. In SYM theories with extended supersymmetry, the $\mathcal{N}$-dependence of the two-loop dilatation operator can be factorized (modulo an additive normalization constant) into a multiplicative c-number. This property makes the eigenspectrum of the two-loop dilatation operator alike in all gauge theories including the maximally supersymmetric theory. Our analysis suggests that integrability is only tied to the planar limit and it is sensitive neither to conformal symmetry nor supersymmetry.
\end{abstract}

\footnotetext{
${ }^{1}$ Unité Mixte de Recherche du CNRS (UMR 8627).
} 


\section{Contents}

1 Introduction 1

2 Case of study 4

3 Representations of the dilatation operator $\quad 8$

3.1 Mixing matrix . . . . . . . . . . . . . . . . . . . . . . 9

3.2 Momentum representation . . . . . . . . . . . . . . . . . . . . 11

4 Dilatation operator: 1-loop 13

4.1 Regularization scheme . . . . . . . . . . . . . . . . . . . . . . . . 13

4.2 One-loop . . . . . . . . . . . . . . . . . . . . . . . . . . . . . . 14

$\begin{array}{lll}5 & \text { Dilatation operator: 2-loops } & 17\end{array}$

5.1 Three-particle contributions . . . . . . . . . . . . . . . . . . . . 19

5.2 Two-particle contributions . . . . . . . . . . . . . . . . . . . 23

5.2 .1 Gluon contribution . . . . . . . . . . . . . . . . . . 23

5.2 .2 Scalar contribution . . . . . . . . . . . . . . . . . . 26

5.2 .3 Two-particle kernel . . . . . . . . . . . . . . . . . . . . . . . . . 27

5.3 One-particle contributions . . . . . . . . . . . . . . . . . . . 30

5.4 Two-loop result . . . . . . . . . . . . . . . . . . . . . . . . . 31

5.4 .1 Scheme dependence . . . . . . . . . . . . . . . . . . . 31

5.4 .2 Limiting cases $\ldots \ldots \ldots \ldots \ldots \ldots \ldots \ldots$

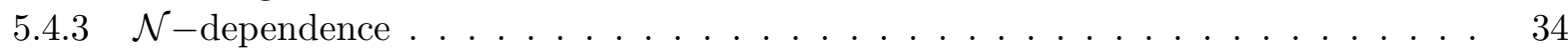

$6 \quad$ Eigenspectrum of the dilatation operator $\quad 35$

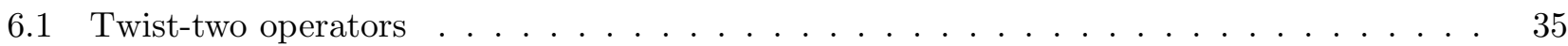

6.1 .1 Eigenspectrum at one-loop . . . . . . . . . . . . . . . . 35

6.1 .2 Conformal svmmetrv . . . . . . . . . . . . . . . . . . . . . . 36

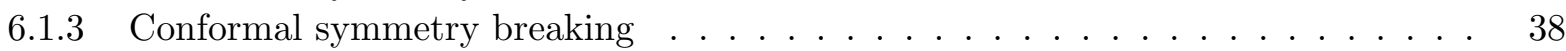

6.1.4 Contribution of scalars from one-loop special conformal anomaly . . . . . . . . . 42

6.1.5 Anomalous dimension of twist-two operators . . . . . . . . . . . . . . 43

6.2 Three-particle operators . . . . . . . . . . . . . . . . . . . . 46

6.2 .1 Eigenspectrum to one-loop . . . . . . . . . . . . . . . . . . 46

6.2 .2 Criterium of higher loop integrability . . . . . . . . . . . . . . . . . . 49

6.2.3 Numerical diagonalization of the two-loop dilatation operaton . . . . . . . . . . . 52

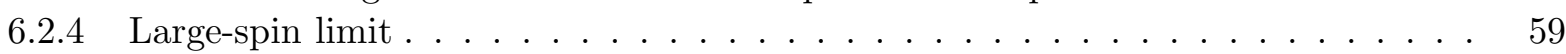

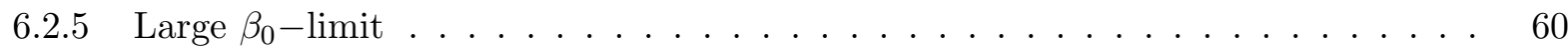

\begin{tabular}{|cc}
7 Discussion and conclusions & 61
\end{tabular}

\begin{tabular}{|cc}
\hline A Forward limit & 63
\end{tabular}

\begin{tabular}{|rr}
\hline B Subtraction terms & 64
\end{tabular} 


\section{Introduction}

There is a growing amount of evidence that four-dimensional gauge theories possess a hidden symmetry. The latter is not seen at the level of the classical Lagrangian and manifests itself through remarkable integrability properties of effective dynamics of the quantum theory. The symmetry has been first discovered in studies of high-energy asymptotics of scattering amplitudes in multi-color QCD [1, 2] which led to the identification of the evolution equation for the partial waves with the Schrödinger equation for the celebrated Heisenberg magnet with spins being generators of the $S L(2, \mathbb{C})$ group. Later, similar integrable structures have been observed in the renormalization group (Callan-Symanzik) equations for multi-particle distributions in QCD [3, 4, 5]. These distributions are given by (nonperturbative) matrix elements of composite Wilson operators built from elementary quark and gluon fields and an arbitrary number of covariant derivatives in the totally symmetric representations of the conformal group $S O(4,2)$. The scale dependence of the distribution amplitudes is driven by the anomalous dimensions of these Wilson operators which, in their turn, are defined as eigenvalues of the dilatation operator in QCD. It was found that the one-loop dilatation operator in QCD in the sector of the so-called maximalhelicity operators can be mapped in the multi-color limit into a Hamiltonian of the Heisenberg magnet with the spin operators in the chain sites defined by the generators of the 'collinear' $S L(2, \mathbb{R})$ subgroup of the full conformal group [3, 4, 5]. The phenomenon of integrability is not merely of academic interest as it has a number of important phenomenological applications in QCD (for a review see [6]).

The above mentioned integrable structures are not specific to QCD. They are also present in generic gauge theories including supersymmetric Yang-Mills models with $\mathcal{N}=1,2,4$ supercharges. Supersymmetry enhances the phenomenon by extending integrability to a larger class of observables. In this context, the maximally supersymmetric $\mathcal{N}=4$ Yang-Mills theory is of a special interest with regards to the AdS/CFT correspondence [7]. The gauge/string duality establishes the correspondence between this superconformal Yang-Mills theory and the type IIB string theory on the $\mathrm{AdS}_{5} \times \mathrm{S}^{5}$ background [7]. In particular, the anomalous dimensions of composite Wilson operators in $\mathcal{N}=4$ gauge theory are mapped into the energies of string excitations in the dual string picture [8, 9, 10, 11]. The string sigma models on the anti-de Sitter space are known to possess an infinite set of conserved currents and thus turn out to be completely integrable. This was shown classically to be the case for the critical $A_{d} S_{5} \times S^{5}$ string action [12, 13. as well as for noncritical sigma models with the anti-de Sitter as a factor of the target space [14], which are dual to particular (non-)supersymmetric Yang-Mills theories. The gauge/string duality hints that these structures should manifest themselves through hidden symmetries of the spectra of anomalous dimensions of Wilson operators in dual gauge theories to all orders of perturbation theory.

At present, all studies firmly point out that the one-loop dilatation operator in certain sectors of QCD and supersymmetric Yang-Mills (SYM) theories with $\mathcal{N}<4$ supercharges possesses integrability properties. In maximally supersymmetric theory the phenomenon gets extended to all Wilson operators [15. A natural question arises whether integrability persists to higher loops as suggested by the gauge/string duality. Recently a great deal of activity has been devoted to the test of higher loop integrability in the $\mathcal{N}=4$ theory and significant evidence has been gathered supporting its presence in various closed 'compact' subsectors specific to this particular theory (see Ref. [16] for a review and Ref. [17] for recent developments). These sectors embrace composite operators with a nontrivial $R$-charge for which the corresponding mixing matrices 
have a finite size determined by the number of constituent fields which build up the former. They do not comprise however Wilson operators involving an arbitrary number of covariant derivatives. ${ }^{1}$ For such operators, the mixing matrices could have an arbitrary large size and they define the 'noncompact' sector. In distinction with the compact sectors, which are intrinsic to models with a nontrivial $R$-symmetry group, the noncompact ones are present in all gauge theories including nonsupersymmetric ones. This offers one an opportunity to compare the properties of the dilatation operators in various gauge theories in the same noncompact sector and identify the origin of the symmetry responsible for the existence of integrability.

In this paper we will study the dilatation operator in the noncompact $S L(2)$ sector for the aligned-helicity fermionic operators in QCD and supersymmetric Yang-Mills theories with $\mathcal{N}=$ 1,2, 4 supercharges. ${ }^{2}$ These operators are constructed from quark/gaugino fields of helicity $+\frac{1}{2}$ belonging to the fundamental/adjoint representation of the gauge group and an arbitrary number of covariant derivatives projected onto the light-cone. To one-loop order, both in QCD and all SYM models in the multi-color limit, the mixing matrix for such operators can be mapped into a Hamiltonian of the Heisenberg spin chain. The length of the spin chain equals the number of fermions which form a given Wilson operator and the spin operators are determined by the generators of the collinear $S L(2 ; \mathbb{R})$ subgroup of full (super)conformal group.

It is well-known that the conformal symmetry is broken in QCD and SYM theories with $\mathcal{N}<4$ supercharges while in the maximally supersymmetric $\mathcal{N}=4$ model it survives on the quantum level. However the conformal anomaly modifies anomalous dimensions starting from two loops only and, therefore, the one-loop dilatation operator inherits the conformal symmetry of the classical theory. Starting from two-loop order, the dilatation operator in the $S L(2)$ sector acquires several new features. First, it receives conformal symmetry breaking corrections arising both due to a nonzero beta-function and a subtle symmetry-violating effect induced by the regularization procedure. ${ }^{3}$ Second, the form of the dilatation operator starts to depend on the representation of the fermion fields, i.e., fundamental $S U(3)$ in $\mathrm{QCD}$ and adjoint $S U\left(N_{c}\right)$ in SYM theories. The difference between the two is that it is only in the latter case that one can select planar diagrams by going over to the multi-color limit, while in the former case the large- $N_{c}$ counting is inapplicable and the two-loop dilatation operator receives equally important contributions from both planar and nonplanar Feynman graphs. Thus, by studying the two-loop dilatation operator in the $S L(2)$ sector we are able to identify what intrinsic properties of gauge theories are responsible for the existence of the integrability phenomenon per se:

- Conformal symmetry

- Supersymmetry

- Planar limit

We are able to give definite answers to all of the raised questions by performing the two-loop calculation of the anomalous dimensions of the aforementioned aligned-helicity fermionic operators in QCD and all SYM theories. The computation is performed within two different renormalization schemes based on dimensional regularization and dimensional reduction. The latter is

\footnotetext{
${ }^{1}$ The exception is the study in Ref. [18 of spin-three scalar operators with three covariant derivatives.

${ }^{2} \mathrm{~A}$ preliminary account of this work was published in a letter [19].

${ }^{3}$ It worth mentioning that in distinction with the compact sectors, the dilatation operator of gauge theories in the $S L(2)$ sector defines one of the component of the spin operator on the spin chain side.
} 
required for preserving supersymmetry in SYM theories. Actually, we find that the anomalous dimensions in the two schemes only differ by an additive constant indicating that the integrability phenomenon (if present) is scheme independent.

Our finding can be summarized as follows. According to Liouville, the dynamical model is integrable provided it acquires enough integrals of motion to match the number of degrees of freedom. For an all-loop dilatation operator $\mathbb{H}(\lambda)$, depending on 't Hooft coupling constant $\lambda$ and acting on a Wilson operator built from $L$ constituent fields and an arbitrary number of covariant derivatives, integrability would require, in general, the existence of $L$ conserved charges. Two of the charges - the light-cone component of the total momentum of $L$ fields and the scaling dimension of the operator - follow immediately from Lorentz covariance of the gauge theory. ${ }^{4}$ However, the identification of the remaining charges $q_{k}(\lambda)$ with $k=3, \ldots, L$ is an extremely nontrivial task. The eigenvalues of the charges $q_{k}$ define the complete set of quantum numbers parameterizing the eigenspectrum of the dilatation operator. Integrability imposes a nontrivial analytical structure of anomalous dimensions of Wilson operators and implies the double degeneracy of eigenvalues with the opposite parity [2, 20, 3. To the lowest order of perturbation theory this property has been verified in Refs. 3, 4] with the help of the Quantum Inverse Scattering Method [21. In particular, it was found there that breaking of integrability leads to lifting of the degeneracy in the eigenspectrum of the one-loop dilatation operator. ${ }^{5}$ We argue that the same relation between integrability and degeneracy of the eigenstates holds true to two loops and allows one to identify high loop corrections to the conserved charges.

Going over through diagonalization of the two-loop dilatation operator, we find that the desired pairing of eigenvalues occurs for three-gaugino operators in SYM theories with $\mathcal{N}=1,2,4$ supercharges and the $S U\left(N_{c}\right)$ gauge group. In that case, nonplanar diagrams provide vanishing contribution and, as a consequence, the exact anomalous dimensions coincide with those in the multi-color limit. The three-quark (baryonic) operators in QCD are well-defined for the $S U(3)$ gauge group only. Due to different color representation, the two-loop dilatation operator in QCD receives corrections from certain nonplanar diagrams which yield breaking of pairing of eigenvalues. To trace the origin of breaking of integrability we split the expression for the twoloop anomalous dimensions into the sum of two gauge invariant terms with one of them depending on the beta-function. The latter term provides conformal symmetry breaking contribution to the dilatation operator both in QCD and SYM theories with $\mathcal{N}=1,2$. As follows from our analysis, the pairing of the eigenstates persists in QCD for terms depending on the beta function, but does not hold for the remaining "conformal" piece.

Starting from two loops, the dilatation operator in SYM theories depends on the number of supercharges $\mathcal{N}$. This dependence comes about through the contribution of $2(\mathcal{N}-1)$ real scalars and $\mathcal{N}$ gaugino fields propagating inside loops. We find that both contributions are irrelevant for enhancement or insuring of integrability since the $\mathcal{N}$-dependence of the two-loop dilatation operator can be factored out (modulo an additive normalization factor) into a multiplicative c-number. This property makes the eigenspectrum of the two-loop dilatation operator alike in all gauge theories including the $\mathcal{N}=4 \mathrm{SYM}$. Thus the main conclusion of our analysis is that the phenomenon of integrability is only tied to the planar limit and is sensitive neither to conformal

\footnotetext{
${ }^{4}$ The two charges commute for the operators with zero total light-cone momentum only, the so-called forward limit.

${ }^{5}$ One should mention however that, in general, integrability does not necessary imply degeneracy in the eigenspectrum. The simplest example is provided by integrable open spin chains which determine the one-loop dilatation operator in multi-color QCD in the sector of mixed antiquark-gluon-quark operators [4, 5].
} 
symmetry nor supersymmetry.

In our subsequent consideration we thoroughly elaborate on this subject. The presentation is organized as follows. In the following section we describe gauge theories under study - QCD and a generic Yang-Mills theory with fermions and scalars belonging to the adjoint representation of the gauge group,- - and specify three-particle operators whose renormalization is scrutinized here. Then in Sect. 3 we work out two equivalent representations of the dilatation operatoras a mixing matrix on the space of local Wilson operators and as an integral operator in the representation of momentum fractions. The former representation is convenient for discussing symmetry properties whereas the latter one is the most appropriate for higher-loop calculations. To start with, we describe in Sect. 4 the main features of the approach on a simpler example of the one-loop dilatation operator. Then, we move on to the two-loop case and compute the irreducible components of the two-loop three-particle kernel term by term in Sect. 5. The reader, who is not interested in intermediate details but only wants to see the final result, is directed straightforwardly to Sect. 5.4. Having computed the two-loop dilatation operator, we turn to the study of its eigenspectrum in Sect. 6. At first, we address in Sect. 6.1 the sector of two-particle (twist-two) operators and diagonalize the dilatation operator at one loop making use of the conformal invariance. Then, we describe the constraints on the form of the dilatation operator imposed by conformal symmetry breaking in gauge theories. In Sect. 6.1.5 we calculate the twoloop anomalous dimensions of twist-two operators in SYM theories with an arbitrary number of supersymmetries $\mathcal{N}$ and demonstrate that all of them are expressed in terms of the beta-function of the underlying gauge theory and the so-called "universal" anomalous dimension. Finally, we turn in Sect. 6.2 to the three-particle dilatation operator. We review the relation between integrability of the one-loop dilatation operator and pairing of its eigenstates and demonstrate that the double degeneracy observed in QCD and all SYM theories to one loop implies the existence of a conserved charge. We extend this consideration to two loops in Sect. 6.2.2 and explicitly construct the set of operators commuting with the dilatation operator and among themselves. We present the two-loop spectra of anomalous dimensions in Sect. 6.2.3 for QCD and all SYM theories and draw conclusions about two-loop integrability. We close this section with presenting two limiting cases in which we are able to reconstruct the all-loop dilatation operator and identify the corresponding integrable structures. Section 7 contains concluding remarks. Two appendices provide technical details of the calculational procedures used in our analysis.

\section{Case of study}

The goal of the present study is to reveal hidden integrability in four-dimensional Yang-Mills theory describing the coupling of gauge fields to fermions/scalars. Depending on the representation in which the latter fields are defined one can distinguish two different types of the gauge theories: QCD and supersymmetric extensions of Yang-Mills theory. In the first case, the gauge fields are coupled to quarks in the fundamental representation of the $S U\left(N_{c}\right)$ gauge group and the QCD Lagrangian is given by a well-known expression

$$
\mathcal{L}_{\mathrm{QCD}}=-\frac{1}{2} \operatorname{tr} F_{\mu \nu} F^{\mu \nu}+i \bar{\psi} \gamma_{\mu} D^{\mu} \psi
$$

Here quarks are described by four-component Dirac fermions $\psi=\left(\lambda_{\alpha}, \bar{\chi}^{\dot{\alpha}}\right)$ and the gauge field strength $F_{\mu \nu}=\frac{i}{g}\left[D_{\mu}, D_{\nu}\right]$ is determined in terms of the covariant derivatives $D_{\mu}=\partial_{\mu}-i g A_{\mu}^{a} t^{a}$ 
with generators $t^{a}$ in the fundamental representation of the $S U\left(N_{c}\right)$ normalized conventionally as $\operatorname{tr}\left(t^{a} t^{b}\right)=\frac{1}{2} \delta^{a b}$. In the second case, the gauge fields are coupled to fermions and scalars belonging to the adjoint representation of the $S U\left(N_{c}\right)$ group. The Lagrangian of the corresponding YangMills theory takes the form

$$
\begin{aligned}
\mathcal{L}_{\text {adj }}=\operatorname{tr}\{ & -\frac{1}{2} F_{\mu \nu} F^{\mu \nu}+2 i \bar{\lambda}_{\dot{\alpha} A} \sigma_{\mu}^{\dot{\alpha} \beta} D^{\mu} \lambda_{\beta}^{A}+\frac{1}{2}\left(D_{\mu} \phi^{A B}\right)\left(D^{\mu} \bar{\phi}_{A B}\right) \\
& \left.-h_{\mathrm{Y}} \lambda^{\alpha A}\left[\bar{\phi}_{A B}, \lambda_{\alpha}^{B}\right]+h_{\mathrm{Y}} \bar{\lambda}_{\dot{\alpha} A}\left[\phi^{A B}, \bar{\lambda}_{B}^{\dot{\alpha}}\right]-h_{4}\left[\phi^{C D}, \bar{\phi}_{A B}\right]\left[\phi^{A B}, \bar{\phi}_{C D}\right]\right\},
\end{aligned}
$$

with the gauge fields, gauginos and scalars, $X=\left\{A_{\mu}, \lambda^{A}, \phi^{A B}\right\}$, all in the adjoint representation of the gauge group, $X=X^{a} t^{a}$ and $A, B=1, \ldots, \mathcal{N}$. The covariant derivative in the adjoint representation is given by $D_{\mu}=\partial_{\mu}-i g\left[A_{\mu},\right]$. The gauginos are described by the Weyl fermion $\lambda^{A}$ which belongs to the fundamental representation of an internal $S U(\mathcal{N})$ symmetry group with its complex conjugate $\bar{\lambda}_{A}=\left(\lambda^{A}\right)^{*}$. The scalars are assembled into the antisymmetric tensor $\phi^{A B}=-\phi^{B A}$, with its complex conjugate $\left(\phi^{A B}\right)^{*}=\bar{\phi}_{A B}$. The supersymmetric Yang-Mills theories with $\mathcal{N}=1,2$ and 4 supercharges are obtained from the Lagrangian (2.2) by adjusting the values of the coupling constants and the number of gaugino and scalar species, $n_{f}$ and $n_{s}$, respectively:

- $\mathcal{N}=1 \mathrm{SYM}$ : there is a single specie of the gaugino, $n_{f}=1$, and no scalars, $n_{s}=0$, so that

$$
h_{\mathrm{Y}}=0, \quad h_{4}=0 .
$$

- $\mathcal{N}=2$ SYM: there are two species of gaugino, $n_{f}=2$, and two real scalars, $n_{s}=2$,

$$
h_{\mathrm{Y}}=g, \quad h_{4}=\frac{1}{16} g^{2}, \quad \phi^{A B}=\sqrt{2} \varepsilon^{A B} \phi .
$$

- $\mathcal{N}=4$ SYM: there are four species of gaugino $n_{f}=4$, and six real scalars, $n_{s}=6$,

$$
h_{\mathrm{Y}}=\sqrt{2} g, \quad h_{4}=\frac{1}{8} g^{2}, \quad \phi^{A B}=\frac{1}{2} \varepsilon^{A B C D} \bar{\phi}_{C D} .
$$

The above relations between the coupling constants are imposed by supersymmetry. It also relates to each other the number of gauginos and scalars

$$
n_{f}=1+\frac{n_{s}}{2}=\mathcal{N} .
$$

One of our findings is that, as we will show in Sect. 5.2.2, integrability is not tied to supersymmetry. In other words, the phenomenon persists in the generic Yang-Mills theory (2.2) for arbitrary $\mathcal{N}$, to two loop order at least.

The classical Lagrangians of the Yang-Mills theories (2.1) and (2.2) enjoy the conformal symmetry which will play an important rôle in our analysis. On the quantum level this symmetry is anomalous (except of $\mathcal{N}=4 \mathrm{SYM}$ ) due to running of the gauge coupling constant,

$$
\frac{d \ln g^{2}}{d \ln \mu}=\beta\left(g^{2}\right)=-\beta_{0} \frac{g^{2}}{8 \pi^{2}}-\beta_{1}\left(\frac{g^{2}}{8 \pi^{2}}\right)^{2}+\mathcal{O}\left(g^{6}\right) .
$$

The beta-function in QCD is given to two loop order by the well-known expression

$$
\beta_{\mathrm{QCD}}\left(g^{2}\right)=-\frac{g^{2}}{8 \pi^{2}}\left(\frac{11}{3} N_{c}-\frac{2}{3} n_{f}\right)-\left(\frac{g^{2}}{8 \pi^{2}}\right)^{2}\left(\frac{17}{3} N_{c}^{2}-C_{F} n_{f}-\frac{5}{3} N_{c} n_{f}\right)+\mathcal{O}\left(g^{6}\right),
$$


where $C_{F}=\left(N_{c}^{2}-1\right) /\left(2 N_{c}\right)$ is the Casimir operator in the fundamental representation of the $S U\left(N_{c}\right)$ group and $n_{f}$ the number of the quark flavors. In supersymmetric Yang-Mills theory with $\mathcal{N}=1,2,4$ supercharges the two-loop beta-function is given by

$$
\beta_{\text {SYм }}\left(g^{2}\right)=-\frac{g^{2} N_{c}}{8 \pi^{2}}(4-\mathcal{N})-\left(\frac{g^{2} N_{c}}{8 \pi^{2}}\right)^{2}(2-\mathcal{N})(4-\mathcal{N})+\mathcal{O}\left(g^{6}\right) .
$$

For $\mathcal{N}=4$ the beta-function vanishes to all orders of perturbation theory and the gauge theory remains conformal on the quantum level. For $\mathcal{N}<4$ the beta-function is nonvanishing and, as a consequence, the conformal symmetry is broken. For $\mathcal{N}=2$ the beta-function receives contribution to one-loop only while for $\mathcal{N}=1$ the beta-function coincides with the QCD expression (2.8) upon substitution $n_{f} \rightarrow N_{c}$ and $C_{F} \rightarrow N_{c}$.

The central object of our study is the scale dependence of the gauge invariant nonlocal operators given by the product of elementary fields $X\left(z n_{\mu}\right)$ "living" on the light-ray defined by a light-like vector $n_{\mu}$ (so that $n_{\mu}^{2}=0$ ). The explicit form of these operators depends on the representation of the $S U\left(N_{c}\right)$ gauge group to which these fields belong. For elementary fields $X=X^{a} t^{a}$ in the adjoint representation one can construct single-trace operators of the length $L$

$$
\mathbb{O}_{\text {adj }}\left(z_{1}, z_{2}, \ldots, z_{L}\right)=\operatorname{tr}\left\{X\left(z_{1} n\right) X\left(z_{2} n\right) \cdots X\left(z_{L} n\right)\right\},
$$

where the gauge invariance is restored by inserting the Wilson lines in between each pair of fields,

$$
\left[z_{j}, z_{j+1}\right]=P \exp \left(i g \int_{z_{j+1}}^{z_{j}} d z n^{\mu} A_{\mu}(z n)\right) .
$$

Later on we will adopt the light-light gauge $(n \cdot A) \equiv A_{+}=0$, so that the gauge links all shrink to the unit matrix. In what follows we do not display them for brevity. For fields $X^{j}$ (quarks) in the fundamental representation, we can introduce a baryon operator

$$
\mathbb{O}_{\text {fun }}\left(z_{1}, z_{2}, \ldots, z_{N_{c}}\right)=\varepsilon_{j_{1} j_{2} \ldots j_{N_{c}}} X^{j_{1}}\left(z_{1} n\right) X^{j_{2}}\left(z_{2} n\right) \cdots X^{j_{N_{c}}}\left(z_{N_{c}} n\right) .
$$

It is built from exactly $N_{c}$ fields and is completely antisymmetric with respect to permutation of any pair of them.

The nonlocal light-cone operators (2.10) and (2.12) have the meaning of generating functions of local gauge invariant Wilson operators of the maximal Lorentz spin. The latter operators can be obtained from Taylor expansion of $\mathbb{O}_{\text {adj }}\left(z_{1}, z_{2}, \ldots, z_{L}\right)$ and $\mathbb{O}_{\text {fun }}\left(z_{1}, z_{2}, \ldots, z_{N_{c}}\right)$ in powers of $z_{i}$. Each field entering (2.10) and (2.12) can be decomposed into different helicity components and, as a consequence, the nonlocal light-cone operators can be classified according to their helicity content. Among them one can distinguish light-cone operators with all helicities aligned. In what follows we shall refer to them as maximal helicity operators.

It is well-known that local Wilson operators mix under renormalization and satisfy the evolution (Callan-Symanzik) equations. The same is true for the light-cone operators (2.10) and (2.12) although the explicit form of the evolution kernels driving the scale dependence of these operators is different due to their nonlocal form. In particular, for nonlocal light-cone operators (2.10) built from the so-called "good" components of fundamental fields the evolution equation 
takes the following form ${ }^{6}[22,23]$

$$
\left(\mu \frac{\partial}{\partial \mu}+\beta\left(g^{2}\right) \frac{\partial}{\partial g^{2}}\right) \mathbb{O}_{\mathrm{adj}}\left(z_{1}, z_{2}, \ldots, z_{L}\right)=-\left[\mathbb{H}_{L} \cdot \mathbb{O}_{\mathrm{adj}}\right]\left(z_{1}, z_{2}, \ldots, z_{L}\right)+\mathcal{O}\left(1 / N_{c}^{2}\right)
$$

where $\mathcal{O}\left(1 / N_{c}^{2}\right)$ denotes terms corresponding to transitions involving splitting of the single trace into multi trace operators, i.e., $\mathbb{O}_{\text {adj }}\left(z_{1}, z_{2}, \ldots, z_{L}\right) \rightarrow \mathbb{O}_{\text {adj }}\left(z_{1}, z_{2}\right) \mathbb{O}_{\text {adj }}\left(z_{3}, \ldots, z_{L}\right)$. The evolution equation (2.13) follows from the Ward identity under dilatation transformations in the underlying gauge theory.

The integral operator $\mathbb{H}_{L}$ entering (2.13) defines a representation of the dilatation operator $\mathbb{D}$ on the space spanned by nonlocal light-cone operators (2.10). To the lowest order of perturbation theory, the explicit form of $\mathbb{H}_{L}$ in Yang-Mills theories with an arbitrary number of supercharges has been found in Ref. [24]. It turns out that to one-loop order, the evolution kernel has a number of remarkable properties in the multi-color limit:

- The length $L$ of the single trace operator is preserved and, as a consequence, $\mathbb{H}_{L}$ can be realized as a quantum mechanical Hamiltonian for a system of $L$ particles with nearestneighbor interaction, $\mathbb{H}_{L}=H_{12}+H_{23}+\ldots+H_{L 1}$;

- $\mathbb{H}_{L}$ reveals a hidden integrability symmetry in the sector of maximal helicity operators both in QCD and in supersymmetric Yang-Mills theories: it possesses exactly $L$ conserved charges and, therefore, is completely integrable;

- $\mathbb{H}_{L}$ can be mapped into a Hamiltonian of the Heisenberg spin chain of length $L$ and spin operators being generators of the $S L(2 \mid \mathcal{N})$ group with $\mathcal{N}$ counting the number of supercharges.

The main question that we would like to address in the present paper is whether the integrability phenomenon just described is preserved to two-loop order and beyond it.

To this end, we shall evaluate two-loop corrections to the evolution kernel $\mathbb{H}_{L}$ and study its properties. Due to different partonic content in Yang-Mills theories with different number of supercharges, the number of maximal helicity light-cone operators varies with $\mathcal{N}$. Calculation of the two-loop evolution kernel for any of these operators is an extremely tedious task. Supersymmetry implies that the evolution kernels corresponding to different maximal helicity operators of the same length $L$ are related to each other. Making use of this property, we shall restrict our analysis to maximal helicity operators built from the helicity $+\frac{1}{2}$ component of the gaugino field $\lambda_{\alpha}^{A}($ with $\alpha=1,2)$

$$
\lambda_{+\alpha}^{A} \equiv \frac{1}{2} \bar{\sigma}^{-}{ }_{\alpha \dot{\beta}} \sigma^{+\dot{\beta} \gamma} \lambda_{\gamma}^{A}=\left(\begin{array}{c}
\lambda^{A} \\
0
\end{array}\right)
$$

It actually possesses a single nonvanishing component $\lambda^{A}=\lambda^{a A} t^{a}$ (with $a=1, \ldots, N_{c}^{2}-1$ and $A=1, \ldots, \mathcal{N})$ which is charged with respect to internal $S U(\mathcal{N})$ isotopic group. Then, one decomposes the product of $L$ fields over irreducible $S U(\mathcal{N})$ components and selects the one with the maximal $R$-charge

$$
\mathbb{O}\left(z_{1}, z_{2}, \ldots, z_{L}\right)=\underset{A_{1} A_{2} \ldots A_{L}}{\mathbf{S}} \operatorname{tr}\left\{\lambda^{A_{1}}\left(z_{1} n\right) \lambda^{A_{2}}\left(z_{2} n\right) \ldots \lambda^{A_{L}}\left(z_{L} n\right)\right\}
$$

\footnotetext{
${ }^{6}$ This equation has been written under tacit assumption that the light-cone operators (2.10) evolve autonomously in the multicolor limit. In general, $\mathbb{H}_{L}$ has a matrix form as the light-cone operators with different partonic content could mix with each other.
} 
where the operation $\mathbf{S}$ stands for symmetrization over the $S U(\mathcal{N})$ indices. The counter-part of the operators (2.15) in QCD is the baryon operator (2.12) built from helicity $\pm \frac{1}{2}$ "good" component of the quark fields

$$
\psi_{+\uparrow}=\frac{1}{4}\left(1-\gamma_{5}\right) \gamma_{-} \gamma_{+} \psi=\left(\begin{array}{c}
q \\
0 \\
0 \\
0
\end{array}\right), \quad \psi_{+\downarrow}=\frac{1}{4}\left(1+\gamma_{5}\right) \gamma_{-} \gamma_{+} \psi=\left(\begin{array}{c}
0 \\
0 \\
0 \\
\bar{q}
\end{array}\right)
$$

which have a single nonvanishing component transforming in the fundamental representation of $S U\left(N_{c}\right)$. Later in the paper, we will encounter the operators of length $L=2$ and $L=3$ :

- For $L=2$, the generating functions of maximal-helicity operators in SYM and QCD read, respectively,

$$
\begin{aligned}
& \mathbb{O}_{\text {adj }}\left(z_{1}, z_{2}\right)=\operatorname{tr}\left[\lambda^{\{A}\left(z_{1} n\right) \lambda^{B\}}\left(z_{2} n\right)\right] \\
& \mathbb{O}_{\text {fun }}\left(z_{1}, z_{2}\right)=\frac{1}{2} \bar{\psi}_{+\downarrow}\left(z_{1} n\right) \gamma_{+} \bar{\gamma}_{\perp} \psi_{+\uparrow}\left(z_{2} n\right)=\bar{q}_{j}^{\dagger}\left(z_{1} n\right) q^{j}\left(z_{2} n\right),
\end{aligned}
$$

where $q^{j}$ and $\bar{q}^{j}$ are independent helicity $\pm \frac{1}{2}$ components of the quark Dirac field $\psi_{+}$and $\bar{\gamma}_{\perp}=\left(\gamma_{1}-i \gamma_{2}\right) / \sqrt{2}$ is the antiholomorphic Dirac matrix.

- For $L=3$, analogous generating functions look like

$$
\begin{aligned}
& \mathbb{O}_{\text {adj }}\left(z_{1}, z_{2}, z_{3}\right)=\underset{A B C}{\mathbf{S}} \operatorname{tr}\left\{\lambda^{A}\left(z_{1} n\right) \lambda^{B}\left(z_{2} n\right) \lambda^{C}\left(z_{3} n\right)\right\}, \\
& \mathbb{O}_{\text {fun }}\left(z_{1}, z_{2}, z_{3}\right)=\varepsilon_{j k l} q^{j}\left(z_{1} n\right) q^{k}\left(z_{2} n\right) q^{l}\left(z_{3} n\right) .
\end{aligned}
$$

We remind that for fermions in the fundamental representation of the $S U\left(N_{c}\right)$ group, the length of the operator (2.12) ought to be $N_{c}$. Therefore, the operator $\mathbb{O}_{\text {fun }}\left(z_{1}, z_{2}, z_{3}\right)$ is well defined only for $N_{c}=3$. The operators $\mathbb{O}_{\text {fun }}\left(z_{1}, z_{2}\right)$ and $\mathbb{O}_{\text {fun }}\left(z_{1}, z_{2}, z_{3}\right)$ have a direct phenomenological significance: their matrix elements determine the transversity distributions in the nucleon [25] and the distribution amplitude of the delta-isobar [26], respectively.

We would like to stress that the choice of the maximal helicity operator is ambiguous. The reason why we prefer to work with the operators built from helicity $+\frac{1}{2}$ fermions (gaugino, quarks) is that the number and complexity of contributing Feynman diagrams is gradually reduced and more importantly we can make use of available two-loop QCD calculations. In addition, as we will show in Sect. 51 the two-loop dilatation operator acts elastically on the maximal helicity operators built from the fermion fields (2.14) and (2.16). In other words, the operators (2.15) evolve autonomously and the corresponding evolution kernel $\mathbb{H}_{L}$ can be realized as a quantum mechanical Hamiltonian. To two-loop order, $\mathbb{H}_{L}$ is given by the sum over two- and three-particle kernels, $H_{k, k+1}$ and $H_{k, k+1, k+2}$, respectively. To identify the explicit form of these kernels it suffices to consider maximal helicity operators of length $L=3$, Eqs. (2.19) and (2.20). Our strategy will be to evaluate the two-loop evolution kernels for the operators (2.19) and (2.20) and, then, draw conclusions about their symmetry properties.

\section{Representations of the dilatation operator}

Before we proceed with actual calculations we would like to summarize general properties of the evolution kernels entering the renormalization group equation for the light-cone operators (2.19) 
and (2.20)

$$
\left(\mu \frac{\partial}{\partial \mu}+\beta\left(g^{2}\right) \frac{\partial}{\partial g^{2}}\right) \mathbb{O}\left(z_{1}, z_{2}, z_{3}\right)=-[\mathbb{H} \cdot \mathbb{O}]\left(z_{1}, z_{2}, z_{3}\right),
$$

where $\mathbb{H}=\mathbb{H}_{L=3}$. The evolution kernel $\mathbb{H}$ admits the perturbative expansion

$$
\mathbb{H}=\lambda \mathbb{H}^{(0)}+\lambda^{2} \mathbb{H}^{(1)}+\mathcal{O}\left(\lambda^{3}\right),
$$

with $\lambda=C g^{2} / 8 \pi^{2}$ and the color factor $C$ depending on the representation of the fermion fields. Its value will be specified in Sect. 4.2.

The central object of our study is the spectral problem for the dilatation operator

$$
\mathbb{H} \Psi_{\boldsymbol{q}}\left(z_{i}\right)=\gamma_{\boldsymbol{q}}(\lambda) \Psi_{\boldsymbol{q}}\left(z_{i}\right) .
$$

Here the eigenfunctions $\Psi_{\boldsymbol{q}}\left(z_{i}\right)$ depend on the light-cone coordinates, $\gamma_{\boldsymbol{q}}(\lambda)$ define the eigenvalues of the dilatation operator and $\boldsymbol{q}$ denotes the complete set of quantum numbers to be specified later on. As we will show in Sect. 6.2, hidden integrability of the dilatation operator leads to a peculiar regularity in the spectrum of $\gamma_{\boldsymbol{q}}(\lambda)$. In gauge theories with unbroken conformal symmetry, $\beta\left(g^{2}\right)=0$, the solution to the evolution equation (3.1) looks like

$$
\mathbb{O}\left(z_{1}, z_{2}, z_{3}\right)=\sum_{\boldsymbol{q}} \Psi_{\boldsymbol{q}}\left(z_{1}, z_{2}, z_{3}\right) \mathcal{O}_{\boldsymbol{q}}(0)
$$

with $\mathcal{O}_{\boldsymbol{q}}(0)$ being local Wilson operators. Substituting this relation into (3.1) one finds that the operators $\mathcal{O}_{\boldsymbol{q}}(0)$ have an autonomous scale dependence and their anomalous dimensions are given by $\gamma_{\boldsymbol{q}}(\lambda)$. In gauge theories with $\beta\left(g^{2}\right) \neq 0$, the conformal anomaly induces the mixing between the operators $\mathcal{O}_{\boldsymbol{q}}(0)$ starting from two loops. It is driven by the additional term in the evolution equation $\beta\left(g^{2}\right) \partial \Psi_{\boldsymbol{q}}\left(z_{i}\right) / \partial g^{2}$ proportional to the beta-function. It is straightforward to write down a solution to the evolution equation in that case but its explicit form is more complicated as compared to the one with $\beta\left(g^{2}\right)=0$.

\subsection{Mixing matrix}

We remind that the nonlocal light-cone operators (2.19) and (2.20) generate infinite towers of local Wilson operators. In particular, for the operator (2.19) one finds

$$
\mathbb{O}\left(z_{1}, z_{2}, z_{3}\right)=\sum_{k_{1}, k_{2}, k_{3} \geq 0}^{\infty} \frac{\left(-i z_{1}\right)^{k_{1}}}{k_{1} !} \frac{\left(-i z_{2}\right)^{k_{2}}}{k_{2} !} \frac{\left(-i z_{3}\right)^{k_{3}}}{k_{3} !} \mathcal{O}_{k_{1} k_{2} k_{3}}(0)
$$

with the Wilson operators

$$
\mathcal{O}_{k_{1} k_{2} k_{3}}(0)=\underset{A B C}{\mathbf{S}} \operatorname{tr}\left\{\left(i D_{+}\right)^{k_{1}} \lambda^{A}(0)\left(i D_{+}\right)^{k_{2}} \lambda^{B}(0)\left(i D_{+}\right)^{k_{3}} \lambda^{C}(0)\right\}
$$

and $D_{+}=(n \cdot D)=\partial_{+}$in the light-like axial gauge $(n \cdot A)=0$. Substituting (3.5) into the evolution equation (3.1) one recovers a conventional renormalization group equation for threeparticle Wilson operators

$$
\left(\mu \frac{\partial}{\partial \mu}+\beta\left(g^{2}\right) \frac{\partial}{\partial g^{2}}\right) \mathcal{O}_{k_{1} k_{2} k_{3}}(0)=-\sum_{l_{1}, l_{2}, l_{3} \geq 0} \mathbb{V}_{k_{1} k_{2} k_{3}}^{l_{1} l_{2} l_{3}} \mathcal{O}_{l_{1} l_{2} l_{3}}(0)
$$


Here the mixing matrix defines a representation of the dilatation operator (3.2) in the basis of polynomials $\left|k_{1}, k_{2}, k_{3}\right\rangle=\left(-i z_{1}\right)^{k_{1}}\left(-i z_{2}\right)^{k_{2}}\left(-i z_{3}\right)^{k_{3}} /\left(k_{1} ! k_{2} ! k_{3} !\right)$

$$
\mathbb{H}\left|l_{1}, l_{2}, l_{3}\right\rangle=\sum_{k_{1}, k_{2}, k_{3} \geq 0} \mathbb{V}_{k_{1} k_{2} k_{3}}^{l_{1} l_{2} l_{3}}\left|k_{1}, k_{2}, k_{3}\right\rangle .
$$

To find the spectrum of the dilatation operator (3.3) one has to solve the spectral problem for the evolution kernel or equivalently diagonalize the mixing matrix

$$
\sum_{l_{1}, l_{2}, l_{3} \geq 0} \mathbb{V}_{k_{1} k_{2} k_{3}}^{l_{1} l_{2} l_{3}} c_{l_{1} l_{2} l_{3}}(\boldsymbol{q})=\gamma_{\boldsymbol{q}}(\lambda) c_{k_{1} k_{2} k_{3}}(\boldsymbol{q}) .
$$

The coefficients $c_{k_{1} k_{2} k_{3}}(\boldsymbol{q})$ define the expansion of the eigenstates $\Psi_{\boldsymbol{q}}\left(z_{i}\right)$ over the basis (3.8)

$$
\Psi_{\boldsymbol{q}}\left(z_{1}, z_{2}, z_{3}\right)=\sum_{k_{1} k_{2} k_{3}} c_{k_{1} k_{2} k_{3}}(\boldsymbol{q}) \frac{\left(-i z_{1}\right)^{k_{1}}}{k_{1} !} \frac{\left(-i z_{2}\right)^{k_{2}}}{k_{2} !} \frac{\left(-i z_{3}\right)^{k_{3}}}{k_{3} !} .
$$

Let us take into account that the mixing can occur between the Wilson operators $\mathcal{O}_{k_{1} k_{2} k_{3}}(0)$ with the same canonical dimension. This implies that the mixing matrix $\mathbb{V}_{k_{1} k_{2} k_{3}}^{l_{2} l_{2} l_{3}}$ has nonvanishing entries only for $k_{1}+k_{2}+k_{3}=l_{1}+l_{2}+l_{3}$. In terms of the evolution kernel, Eq. (3.8), this property states that the evolution kernel preserves the overall power of the polynomials $\left|k_{1}, k_{2}, k_{3}\right\rangle$, or equivalently

$$
\left[\mathbb{H}, z_{1} \partial_{z_{1}}+z_{2} \partial_{z_{2}}+z_{3} \partial_{z_{3}}\right]=0 .
$$

In addition, due to Poincaré covariance, the anomalous dimension of the operators $\mathcal{O}_{k_{1} k_{2} k_{3}}(x)$ does not depend on the coordinate $x$. In terms of the evolution kernel, this implies that $\mathbb{H}$ has to be translation invariant

$$
\left[\mathbb{H}, \partial_{z_{1}}+\partial_{z_{2}}+\partial_{z_{3}}\right]=0 .
$$

Since the dilatations and translations do not commute with each other, the eigenstates can not diagonalize the two charges simultaneously unless the total light-cone momentum equals zero, $\left(\partial_{z_{1}}+\partial_{z_{2}}+\partial_{z_{3}}\right) \Psi_{\boldsymbol{q}}\left(z_{i}\right)=0$.

Putting $z_{1}=z_{2}=z_{3}=0$ in (3.5) one obtains a local operator with no derivatives

$$
\mathbb{O}(0,0,0)=\mathcal{O}_{000}(0)=\frac{i}{4} f^{a b c} \underset{A B C}{\mathbf{S}} \lambda^{a A}(0) \lambda^{b B}(0) \lambda^{c C}(0) .
$$

This operator evolves autonomously and according to (3.7) and (3.8) its anomalous dimension defines the eigenvalue of the dilatation operator

$$
\mathbb{H}|0,0,0\rangle=3 \Gamma(\lambda)|0,0,0\rangle,
$$

where the additional factor 3 was inserted for the later convenience. As we will show in Sect. 6.2.3, the anomalous dimension

$$
\Gamma(\lambda)=\lambda \Gamma^{(0)}+\lambda^{2} \Gamma^{(1)}+\mathcal{O}\left(\lambda^{3}\right)
$$

sets up the lower/upper bound in the spectrum of three-particle dilatation operators (3.2). 


\subsection{Momentum representation}

The evolution operator $\mathbb{H}$ acts along the light-cone direction $n_{\mu}$ in Minkowski space-time. Yet another representation of $\mathbb{H}$ can be obtained by going over from the configuration to the reciprocal, momentum space. To this end, one performs Fourier transformation of the light-cone operators

$$
\mathbb{O}\left(z_{1}, z_{2}, z_{3}\right)=\int_{-\infty}^{\infty} d u_{1} \int_{-\infty}^{\infty} d u_{2} \int_{-\infty}^{\infty} d u_{3} \mathrm{e}^{i u_{1} z_{1}+i u_{2} z_{2}+i u_{3} z_{3}} \widetilde{\mathbb{O}}\left(u_{1}, u_{2}, u_{3}\right)
$$

with $u_{i}$ having the meaning of light-cone momenta of particles. Substituting (3.5) into this relation one finds that the local Wilson operators are given by the moments of $\widetilde{\mathbb{O}}\left(u_{1}, u_{2}, u_{3}\right)$

$$
\mathcal{O}_{k_{1} k_{2} k_{3}}(0)=\int d u_{1} d u_{2} d u_{3} u_{1}^{k_{1}} u_{2}^{k_{2}} u_{3}^{k_{3}} \widetilde{\mathbb{O}}\left(u_{1}, u_{2}, u_{3}\right) .
$$

In the momentum representation, the Callan-Symanzik equation is known in QCD as the Brodsky-Lepage equation [26]

$$
\left(\mu \frac{\partial}{\partial \mu}+\beta\left(g^{2}\right) \frac{\partial}{\partial g^{2}}\right) \widetilde{\mathbb{O}}\left(u_{1}, u_{2}, u_{3}\right)=\int[d v]_{3} \mathbb{V}\left(u_{1}, u_{2}, u_{3} \mid v_{1}, v_{2}, v_{3}\right) \widetilde{\mathbb{O}}\left(v_{1}, v_{2}, v_{3}\right),
$$

where the integration measure is

$$
[d v]_{3} \equiv d v_{1} d v_{2} d v_{3} \delta\left(\sum_{j=1}^{3} v_{j}-\sum_{j=1}^{3} u_{j}\right) .
$$

The evolution kernel in the momentum representation, $\mathbb{V}$, is obtained from $\mathbb{H}$ through the Fourier transform

$$
\mathbb{H} \cdot \mathrm{e}^{-i \sum_{k} v_{k} z_{k}}=-\int[d u]_{3} \mathbb{V}(\boldsymbol{u} \mid \boldsymbol{v}) \mathrm{e}^{-i \sum_{k} u_{k} z_{k}},
$$

with $[d u]_{3}$ determined by Eq. (3.19) with $u_{i}$ and $v_{i}$ interchanged. According to (3.12), the evolution kernel preserves the total momentum $v_{1}+v_{2}+v_{3}=u_{1}+u_{2}+u_{3}$. This property is automatically preserved in (3.18). In addition, it follows from (3.11) that the evolution kernel is a homogeneous function of the momentum fractions

$$
\mathbb{V}\left(\lambda u_{1}, \lambda u_{2}, \lambda u_{3} \mid \lambda v_{1}, \lambda v_{2}, \lambda v_{3}\right)=\lambda^{-2} \mathbb{V}\left(u_{1}, u_{2}, u_{3} \mid v_{1}, v_{2}, v_{3}\right) .
$$

Obviously, the perturbative structure of the evolution kernel $\mathbb{V}$ is identical to the one introduced for $\mathbb{H}$, Eq. (3.2).

Combining together (3.20) and (3.8) one obtains the following representation for the mixing matrix

$$
\int[d u]_{3} \mathbb{V}(\boldsymbol{u} \mid \boldsymbol{v}) u_{1}^{k_{1}} u_{2}^{k_{2}} u_{3}^{k_{3}}=-\sum_{\substack{l_{1}, l_{2}, l_{3} \geq 0 \\ \sum k_{i}=\sum l_{j}}} \mathbb{V}_{k_{1} k_{2} k_{3}}^{l_{1} l_{2} l_{3}} v_{1}^{l_{1}} v_{2}^{l_{2}} v_{3}^{l_{3}}
$$

that is, positive integer moments of the evolution kernel with respect to $u$-variables are polynomials in $v$-variables of the same degree $k_{1}+k_{2}+k_{3}=l_{1}+l_{2}+l_{3}$. Below this property will play an important role in our analysis and we shall refer to it as the polynomiality condition. 
By construction, the eigenvalues of the evolution kernel $\mathbb{V}(\boldsymbol{u} \mid \boldsymbol{v})$ defined in (3.20) coincide with $\gamma_{\boldsymbol{q}}(\lambda)$, Eq. (3.3). To see this, one considers the spectral problem for the mixing matrix

$$
\sum_{k_{1}, k_{2}, k_{3} \geq 0} w^{k_{1} k_{2} k_{3}}(\boldsymbol{q}) \mathbb{V}_{k_{1} k_{2} k_{3}}^{l_{1} l_{2} l_{3}}=\gamma_{\boldsymbol{q}}(\lambda) w^{l_{1} l_{2} l_{3}}(\boldsymbol{q}) .
$$

Comparing this relation with (3.9) one observes that the coefficients $w^{l_{1} l_{2} l_{3}}(\boldsymbol{q})$ and $c_{k_{1} k_{2} k_{3}}(\boldsymbol{q})$ are, respectively, the left and right eigenstates of the same mixing matrix corresponding to the same eigenvalue $\gamma_{\boldsymbol{q}}(\lambda)$. Introducing the polynomial

$$
P_{\boldsymbol{q}}\left(u_{i}\right)=\sum_{k_{1}, k_{2}, k_{3} \geq 0} w^{k_{1} k_{2} k_{3}}(\boldsymbol{q}) u_{1}^{k_{1}} u_{2}^{k_{2}} u_{3}^{k_{3}}
$$

one finds from (3.22) that it diagonalizes the evolution kernel

$$
\int[d u]_{3} \mathbb{V}(\boldsymbol{u} \mid \boldsymbol{v}) P_{\boldsymbol{q}}\left(u_{i}\right)=-\gamma_{\boldsymbol{q}}(\lambda) P_{\boldsymbol{q}}\left(v_{i}\right)
$$

The left and right eigenstates of the mixing matrix are orthogonal to each other. Being written in terms of the polynomials (3.10) and (3.24) this condition reads

$$
\left.P_{\boldsymbol{q}}\left(\partial_{z_{1}}, \partial_{z_{2}}, \partial_{z_{3}}\right) \Psi_{\boldsymbol{q}^{\prime}}\left(z_{1}, z_{2}, z_{3}\right)\right|_{z_{i}=0} \sim \delta_{\boldsymbol{q \boldsymbol { q } ^ { \prime }}} .
$$

Combining this relation together with (3.4) one concludes that the $P$-polynomials determine the explicit form of local Wilson operators entering (3.4)

$$
\mathcal{O}_{\boldsymbol{q}}(0)=\left.P_{\boldsymbol{q}}\left(\partial_{z_{1}}, \partial_{z_{2}}, \partial_{z_{3}}\right) \mathbb{O}\left(z_{1}, z_{2}, z_{3}\right)\right|_{z_{i}=0}
$$

Moreover, it follows from (3.21) that the eigenstates of the evolution kernel $\mathbb{V}(\boldsymbol{u} \mid \boldsymbol{v})$ have to be homogeneous polynomials

$$
P_{\boldsymbol{q}}\left(\lambda u_{1}, \lambda u_{2}, \lambda u_{3}\right)=\lambda^{N} P_{\boldsymbol{q}}\left(u_{1}, u_{2}, u_{3}\right) .
$$

Here, integer $N \geq 0$ counts the total number of light-cone derivatives in the expression for the Wilson operator (3.27). It also defines the Lorentz spin of $\mathcal{O}_{\boldsymbol{q}}(0)$. The mixing between the operators with different $N$ is protected by Lorentz symmetry and, therefore, $N$ can be identified as one of the quantum numbers $\boldsymbol{q}$.

In this section we described the dilatation operator in two different representations - as a mixing matrix for local Wilson operators and as an integral operator in the representation of momentum fractions. Obviously, the spectrum of the dilatation operator does not depend on the particular representation and its choice is a question of convenience. As we will demonstrate in Sect. 5. the momentum representation has a number of advantages as far as the two-loop calculation of the evolution kernels is concerned. Firstly, by virtue of Lorentz covariance, the eigenvalues of the evolution kernel $\mathbb{V}(\boldsymbol{u} \mid \boldsymbol{v})$ do not depend on the total light-cone momenta $\sum_{j} u_{j}=\sum_{j} v_{j}$. This allows one to put its value equal to zero. From the point of view of nonlocal operators, this corresponds to assuming translation invariance of $\mathbb{O}\left(z_{1}, z_{2}, \ldots, z_{L}\right)$ along the light-cone, or, equivalently, to considering forward matrix elements of the nonlocal light-cone operators in (3.16). In this way, one automatically eliminates the contribution of local operators with total derivatives in (3.5), which in turn simplifies significantly the form of the mixing matrix (3.7). Secondly, there exist well developed techniques for calculating the renormalization group kernels in the momentum representation. We shall profit from higher-order results available in the literature. 


\section{Dilatation operator: 1-loop}

Let us now turn to the computation of the evolution kernel $\mathbb{H}$ for the maximal helicity operators (2.19) and (2.20) both in QCD and supersymmetric Yang-Mills theory. To start with, we review in this section the one-loop calculation of $\mathbb{H}$. It will serve as an illustration of the general formalism which will then be used for more involved two-loop analysis.

\subsection{Regularization scheme}

Performing the calculation we shall adopt the light-like axial gauge $(n \cdot A(x))=A_{+}(x)=0$. Since the evolution operator $\mathbb{H}$ is a gauge-invariant quantity, any choice of gauge fixing is possible. In covariant gauges, the nonlocal light-cone operators (2.10) involve additional gauge links (2.11) between the elementary constituent fields. This leads to proliferation of the number of Feynman diagrams contributing to $\mathbb{H}$. The light-like axial gauge $A_{+}(x)=0$ is advantageous as the number of relevant graphs is much smaller, especially at higher loops. Yet another advantage of this choice is that we can employ certain results for one- and two-loop evolution kernels in QCD available in the literature.

The Yang-Mills theory in the light-like axial gauge has a number of subtleties [27]. The gauge field propagator $\left\langle 0\left|T A_{\mu}^{a}(x) A_{\nu}^{b}(y)\right| 0\right\rangle=(-i) \delta^{a b} D_{\mu \nu}(x-y)$ is given in the momentum representation by

$$
D_{\mu \nu}(k)=\frac{d_{\mu \nu}(k)}{k^{2}+i 0}, \quad d_{\mu \nu}(k)=g_{\mu \nu}-\frac{k_{\mu} n_{\nu}+k_{\nu} n_{\mu}}{k_{+}},
$$

with $k_{+}=(k \cdot n)$. It has a spurious singularity at $k_{+}=0$ which indicates that the gauge ambiguity is not fixed completely for the choice $A_{+}(x)=0$. To give the meaning to the gluon propagator (4.1), one has to specify the prescription for integrating around the pole $k_{+}=0$ inside momentum Feynman integrals. The only available residual gauge fixing which is consistent with causality properties of Feynman integrals is the one due to Mandelstam and Leibbrandt [28, 29]

$$
\frac{1}{\left[k_{+}\right]_{\mathrm{ML}}}=\frac{k_{-}}{k_{+} k_{-}+i 0}
$$

It has been checked for various gauge invariant quantities that to two-loop accuracy this prescription leads to the same results as the Feynman gauge. However, the calculations within the Mandelstam-Leibbrandt prescription are rather involved and there exist another popular choice, the so-called principal value prescription, defined as 30]

$$
\frac{1}{\left[k_{+}\right]_{\mathrm{PV}}}=\frac{1}{2}\left(\frac{1}{k_{+}+i \delta}+\frac{1}{k_{+}-i \delta}\right),
$$

with $\delta \rightarrow 0$. This prescription has proved to be viable and robust in higher order QCD calculations of the evolution kernels and we will accept it for two-loop calculations in our subsequent analysis.

To isolate divergences of Feynman integrals we will employ in parallel two different regularization schemes - dimensional reduction (DRED) and dimensional regularization (DREG). In both schemes one regularizes Feynman integrals by setting the space-time dimension to $D=4-2 \varepsilon$ and taking the limit $\varepsilon \rightarrow 0$ afterwards. In the DRED scheme one keeps the number of all tensor fields components (gauge bosons and fermions) to be the same as in four dimensions [31] while 
in the DREG scheme this number is $\varepsilon$-dependent. The two schemes are equally suitable for two-loop calculations but the usage of the DRED is mandatory as long as one wants to preserve the supersymmetry of Yang-Mills theory.

To determine the evolution kernel $\mathbb{H}$ we shall examine perturbative corrections to the matrix elements of nonlocal light-cone operators (2.19) and (2.20). For $D \neq 4$, the ultraviolet divergences manifest themselves as poles in $\varepsilon$ and we shall renormalize "bare" nonlocal light-cone operators using the modified minimal subtraction procedure. Depending on the choice of the DREG or DRED regularization schemes, it will be denoted as $\overline{\mathrm{MS}}$ and $\overline{\mathrm{DR}}$, respectively. The renormalized operator is defined as

$$
\mathbb{O}^{\mathrm{R}}\left(z_{1}, z_{2}, z_{3}\right)=\mathbb{Z} \cdot \mathbb{O}^{\text {(bare) }}\left(z_{1}, z_{2}, z_{3}\right), \quad \mathbb{Z}\left(1 / \varepsilon, g^{2}\right)=1+\sum_{n=1}^{\infty} \frac{\mathbb{Z}^{[n]}\left(g^{2}\right)}{\varepsilon^{n}}
$$

with the subtraction "constants" $\mathbb{Z}^{[n]}\left(g^{2}\right)$ chosen in such a manner that the Green functions $\left\langle 0\left|\mathbb{O}^{\mathrm{R}}\left(z_{1}, z_{2}, z_{3}\right) \ldots\right| 0\right\rangle$ remain finite for $\varepsilon \rightarrow 0 .^{7}$ Substituting (4.4) into the evolution equation (2.13) one finds that the evolution operator is related to the residue of the simple $1 / \varepsilon$ pole

$$
\mathbb{H}=-\frac{d \ln \mathbb{Z}\left(1 / \varepsilon, g^{2}\right)}{d \ln \mu}=\frac{d}{d \ln g} \mathbb{Z}^{[1]}\left(g^{2}\right) .
$$

Here in the second relation we used the fact that the gauge coupling constant acquires a nontrivial dimension for $\varepsilon \neq 0$ and, as a consequence, the beta-function in the $D$-dimensional Yang-Mills theory possesses an additional $\varepsilon$-dependent term

$$
\frac{d \ln g^{2}}{d \ln \mu}=\beta_{\varepsilon}\left(g^{2}\right)=-2 \varepsilon+\beta\left(g^{2}\right) .
$$

As we will explain in detail in section 6.1.3, the presence of this term has far reaching consequences for the properties of the dilatation operator. In particular, it implies that in Yang-Mills theory with $\beta\left(g^{2}\right)=0$ the conformal symmetry is broken for $\varepsilon \neq 0$.

We would like to stress that the renormalization constants in the $\overline{\mathrm{MS}}-$ and $\overline{\mathrm{DR}}-$ renormalization schemes are different but they are related to each other though a finite renormalization. As a consequence, the evolution operator depends on the renormalization scheme and one has to distinguish the operators $\mathbb{H}_{\overline{\mathrm{MS}}}$ and $\mathbb{H}_{\overline{\mathrm{DR}}}$. As we will show in Sect. 5.4.1, these operators differ starting from two-loop order only and the difference amounts to a c-number. As a result, if the dilatation operator possesses a hidden symmetry it will hold independently of the chosen renormalization procedure.

\subsection{One-loop}

Examining Feynman diagrams contributing to $\mathbb{O}\left(z_{1}, z_{2}, z_{3}\right)$ it is easy to see that to the $k^{\text {th }}$ order in $\lambda$ the interaction could occur between $(k+1)$ particles at most. As a consequence, to oneloop order $\mathbb{H}^{(0)}$ receives contribution from pair-wise interactions as well as their self-energies. To determine the one-loop evolution kernel, one has to calculate one-loop corrections to the nonlocal light-cone operator $\mathbb{O}\left(z_{1}, z_{2}, z_{3}\right)$, renormalize it according to (4.4) and, finally, evaluate $\mathbb{H}^{(0)}$ using

\footnotetext{
${ }^{7}$ It worth mentioning that the renormalization constants entering 4.4 are certain integral operators acting on the light-cone coordinates of $\mathbb{O}^{\text {(bare) }}\left(z_{1}, z_{2}, z_{3}\right)$.
} 


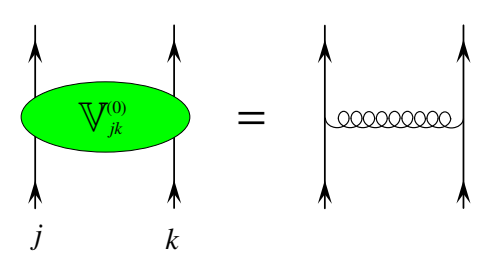

(a)

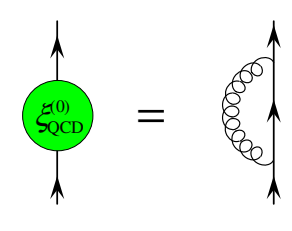

(b)

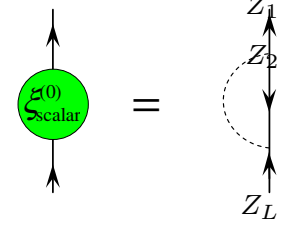

(c)

Figure 1: One-loop diagrams in the light-cone gauge contributing to the pair-wise kernel $(a)$ and the self-energy due to gluons $(b)$ and scalars $(c)$.

(4.5). We sketch below the intermediate steps while the details of the calculation can be found in Refs. [24, 32].

Since to one-loop order the interaction can only occur between two fermions, the dilatation operator has a pair-wise structure

$$
\mathbb{H}^{(0)}=\mathbb{H}_{12}^{(0)}+\mathbb{H}_{23}^{(0)}+\mathbb{H}_{31}^{(0)}+3 \Gamma^{(0)} .
$$

Here the two-particle kernel $\mathbb{H}_{j k}^{(0)}$ acts locally on the $j^{\text {th }}$ and $k^{\text {th }}$ fields while $\Gamma^{(0)}$ receives contribution from the self-energy corrections to the fermion. They are determined by the Feynman diagrams shown in Fig. 1. Notice that in the SYM theory, the self-energy diagrams involve both gauge field and scalar exchanges while the diagram for $\mathbb{H}_{j k}^{(0)}$ contains solely the gluon exchange. The reason for this is the following. One can straightforwardly deduce from the Lagrangian (2.2) that the scalar exchange corresponds to the transition $\lambda^{\{A} \lambda^{B\}} \rightarrow \bar{\lambda}_{C} \bar{\lambda}_{D}$ (symmetrized over $A$ and $B$ ) involving the scalar propagator $\left\langle\phi^{A C} \phi^{B D}\right\rangle$. As follows from (2.2), this propagator vanishes for $\mathcal{N}=1$ and $\mathcal{N}=2$, while for $\mathcal{N}=4$ it is proportional to $\sim \varepsilon^{A B C D}$ and vanishes upon symmetrization over the isotopic indices $A$ and $B$.

The contribution of the diagram in Fig. I(a) to the one-loop dilatation operator in QCD and SYM only differs by an overall color factor. For gauginos in the adjoint representation, the color factor equals $T_{j}^{a} T_{k}^{a}$ with $\left(T_{j}\right)_{b c}^{a}=i f^{a b c}$ being the $S U\left(N_{c}\right)$ generators in the adjoint representation acting on the color indices of the $j^{\text {th }}$ particle. Since the three-fermion operators have zero total color charge, one has $\sum_{j=1,2,3} T_{j}^{a}=0$ and, therefore,

$$
T_{j}^{a} T_{k}^{a}=\frac{1}{2}\left(T_{l}^{a}\right)^{2}-\frac{1}{2}\left(T_{j}^{a}\right)^{2}-\frac{1}{2}\left(T_{k}^{a}\right)^{2}=-\frac{1}{2} N_{c},
$$

with $T_{l}^{a}=-\left(T_{j}+T_{k}\right)^{a}$ and $\left(T_{j}^{a}\right)^{2}=N_{c}$ being the Casimir operator in the adjoint representation of the $S U\left(N_{c}\right)$. For quarks in the fundamental representation of the $S U\left(N_{c}\right)$, the color factor equals

$$
\varepsilon_{i_{1} \ldots i_{j}^{\prime} \ldots i_{k}^{\prime} \ldots i_{N_{c}}}\left(t^{a}\right)_{i_{j}^{\prime} i_{j}}\left(t^{a}\right)_{i_{k}^{\prime} i_{k}}=\varepsilon_{i_{1} \ldots i_{j} \ldots i_{k} \ldots i_{N_{c}}}\left(-\frac{N_{c}+1}{2 N_{c}}\right),
$$

where one takes into account the identity $\left(t^{a}\right)_{j k}\left(t^{a}\right)_{n m}=\delta_{j m} \delta_{k n} / 2-\delta_{j k} \delta_{n m} /\left(2 N_{c}\right)$. We remind that the baryon operator (2.20) is only well-defined for $N_{c}=3$ in which case $-\left(N_{c}+1\right) /\left(2 N_{c}\right)=-2 / 3$. As a result, the contribution of the diagram in Fig. I(a) to the one-loop evolution kernel for the light-cone operators (2.19) and (2.20) is accompanied by the prefactors

$$
\lambda_{\text {fun }}=\frac{g^{2}}{8 \pi^{2}} \frac{4}{3}, \quad \lambda_{\text {adj }}=\frac{g^{2}}{8 \pi^{2}} N_{c},
$$


correspondingly. In what follows we shall use $\lambda_{\text {fun }}$ and $\lambda_{\text {adj }}$ as parameters of the perturbative expansion of the dilatation operator (3.2). In this case, the two-particle kernel $\mathbb{H}_{j k}^{(0)}$ takes the same form for the light-cone operators in the adjoint, Eq. (2.19), and fundamental, Eq. (2.20), representations

$$
\left[\mathbb{H}_{12}^{(0)} \cdot \mathbb{O}\right]\left(z_{1}, z_{2}, z_{3}\right)=\int_{0}^{1} \frac{d \alpha}{\alpha} \bar{\alpha}^{2 j-1}\left[2 \mathbb{O}\left(z_{1}, z_{2}, z_{3}\right)-\mathbb{O}\left(\bar{\alpha} z_{1}+\alpha z_{2}, z_{2}, z_{3}\right)-\mathbb{O}\left(z_{1}, \alpha z_{1}+\bar{\alpha} z_{2}, z_{3}\right)\right]
$$

where $\bar{\alpha} \equiv 1-\alpha$ and $j=1$ is the conformal spin of the quark/gaugino. For the later use, we shall display the dependence of the evolution kernels on the conformal spin $j$. According to Eq. (4.11), the operator $\mathbb{H}_{n k}^{(0)}$ has a transparent physical interpretation: it displaces $n^{\text {th }}$ and $k^{\text {th }}$ particles along the light-cone in the direction of each other.

The gauge field contribution to the self-energy shown in Fig. 1(b) is proportional to the quadratic Casimir operator: $C_{A}=N_{c}$ for the gaugino in the adjoint representation of the $S U\left(N_{c}\right)$ and $C_{F}=\left(N_{c}^{2}-1\right) /\left(2 N_{c}\right)=4 / 3$ for the quark in the fundamental representation of the $S U\left(N_{c}=\right.$ $3)$ group. In addition, in the SYM theory one has to include the contribution of scalars to gaugino self-energy (see in Fig. 1(c)) which is proportional to $\sim n_{s}=2(\mathcal{N}-1)$. Combining together the two contributions one obtains the following expressions for the normalization constant $\Gamma^{(0)}$ in (4.7)

$$
\Gamma_{\mathrm{QCD}}^{(0)}=\frac{1}{2}, \quad \Gamma_{\mathrm{SYM}}^{(0)}=\frac{1}{2} \mathcal{N} .
$$

Notice that $\Gamma_{\mathrm{SYM}}^{(0)}$ coincides with the QCD expression for $\mathcal{N}=1$. Combining together (4.7), (4.11) and (4.12), one obtains the one-loop dilatation operator for the three-particle quark and gaugino operators in QCD and SYM theories, respectively, in the coordinate representation. As follows from (4.11), the two-particle kernel annihilates the local operator $\mathbb{O}(0,0,0)$ and, therefore, $3 \Gamma_{\text {SYM }}^{(0)}$ determines the anomalous dimension of the local gaugino operator (3.13), in agreement with (3.14).

Let us transform the obtained expression for $\mathbb{H}^{(0)}$ into the momentum representation. Applying (3.20) and performing the Fourier transformation one finds that $\mathbb{V}(\boldsymbol{u} \mid \boldsymbol{v})$ has the same structure as (4.7)

$$
\begin{aligned}
\mathbb{V}^{(0)}(\boldsymbol{u} \mid \boldsymbol{v}) & =\left[\mathbb{V}^{(0)}\left(u_{1}, u_{2} \mid v_{1}, v_{2}\right)\right]_{+} \\
& +\left[\mathbb{V}^{(0)}\left(u_{2}, u_{3} \mid v_{2}, v_{3}\right)\right]_{+}+\left[\mathbb{V}^{(0)}\left(u_{3}, u_{1} \mid v_{3}, v_{1}\right)\right]_{+}-3 \Gamma^{(0)} \delta\left(u_{1}-v_{1}\right) \delta\left(u_{2}-v_{2}\right)
\end{aligned}
$$

with the two-particle kernel given by a well-known expression [22, 33]

$$
\mathbb{V}^{(0)}\left(u_{1}, u_{2} \mid v_{1}, v_{2}\right)=\left[\left(\frac{u_{1}}{v_{1}}\right)^{2 j-1} \frac{\Theta\left(u_{1}, v_{1}\right)}{v_{1}-u_{1}}+\left(\frac{u_{2}}{v_{2}}\right)^{2 j-1} \frac{\Theta\left(u_{2}, v_{2}\right)}{v_{2}-u_{2}}\right] \delta\left(u_{1}+u_{2}-v_{1}-v_{2}\right) .
$$

Here the notation was introduced for a generalized "step" function

$$
\Theta\left(u_{n}, v_{n}\right)=\theta\left(u_{n}\right) \theta\left(v_{n}-u_{n}\right)-\theta\left(-u_{n}\right) \theta\left(u_{n}-v_{n}\right) .
$$

The symbol $[\ldots]_{+}$stands for the plus-distribution

$$
[\varphi(u, v)]_{+} \equiv \varphi(u, v)-\delta(u-v) \int d u^{\prime} \varphi\left(u^{\prime}, v\right)
$$


with $\varphi(u, v)$ being a test function. This distribution regularizes the end-point singularity of (4.14) for $v_{i} \rightarrow u_{i}$ in such a way that the evolution kernel (4.13) has finite moments (3.22). Notice that due to the total momentum conservation, $\sum u_{n}=\sum v_{n}$, Eq. (3.19), the two-particle evolution kernel (4.14) preserves the momentum of the third particle, $u_{3}=v_{3}$. By the same token, the constant term in the evolution kernel (4.13) preserves the momenta of all particles, $u_{n}=v_{n}$ with $n=1,2,3$.

The variables $v_{i}$ and $u_{i}$ have the meaning of the light-cone momenta of particles. Calculating the moments of the evolution kernel (3.22), one can assign to $v_{i}$ arbitrary real values. For given $v_{i}$, the integration region over the $u$-variables is not arbitrary and it is determined by the step functions (4.14). It is instructive to consider a special case, the so-called Brodsky-Lepage (BL) limit [26],

$$
0 \leq v_{1}, v_{2}, v_{3} \leq 1, \quad v_{1}+v_{2}+v_{3}=1
$$

It is easy to see that the step function reduces in this limit to $\Theta\left(u_{i}, v_{i}\right) \stackrel{\mathrm{BL}}{=} \theta\left(u_{i}\right) \theta\left(v_{i}-u_{i}\right)$ and, as a consequence,

$$
\begin{aligned}
{\left[\mathbb{V}^{(0)}\left(u_{1}, u_{2} \mid v_{1}, v_{2}\right)\right]_{+} \stackrel{\mathrm{BL}}{=} } & {\left[\left(\frac{u_{1}}{v_{1}}\right)^{2 j-1} \frac{\theta\left(u_{1}\right) \theta\left(v_{1}-u_{1}\right)}{v_{1}-u_{1}}\right.} \\
& \left.+\left(\frac{u_{2}}{v_{2}}\right)^{2 j-1} \frac{\theta\left(u_{2}\right) \theta\left(v_{2}-u_{2}\right)}{v_{2}-u_{2}}\right]_{+} \delta\left(u_{1}+u_{2}-v_{1}-v_{2}\right) .
\end{aligned}
$$

Substituting this expression into (4.13), one verifies that the possible values of the $u$-variables are restricted to the same simplex as in (4.16)

$$
0 \leq u_{1}, u_{2}, u_{3} \leq 1, \quad u_{1}+u_{2}+u_{3}=1
$$

This property has a simple physical interpretation. It implies that for all three particles moving forward in time along the light-cone direction $n_{\mu}$, the interaction described by the evolution kernel (4.13) can turn none of the particles to propagate backward in time. Remarkably enough, this property is general enough to hold to all order of perturbation theory [34, 35. Below in Sect. 5 we will demonstrate its validity to two loops. In the following exposition we will stick to the BL-representation since, on the one hand, it simplifies the step-function structure of the momentum space dilatation operator and, on the other hand, suffices for evaluation of anomalous dimension mixing matrix. A simple procedure [36] for reconstruction of the complete evolution operator without the restriction to the simplex (4.16) is given in Appendix A.

\section{Dilatation operator: 2-loops}

In the previous section we have calculated the one-loop evolution kernel for the three-particle gaugino/quark operators (2.19) and (2.20). We demonstrated that it is given by the same, universal expression (4.11) both in QCD and in SYM theories with arbitrary number of supercharges $\mathcal{N}$. The fact that the fermions are defined in different representations of the gauge group manifests itself in the different color factors accompanying the coupling constant, Eq. (4.10). Let us now extend our analysis to two loops. As we will see, the above mentioned universality gets lost beyond leading order and one has to consider separately the gaugino and quark operators. In the former case, we will start with the $\mathcal{N}=1$ SYM theory and will gradually increase the diversity 
of the particle's content by going over to $\mathcal{N}=2$ and $\mathcal{N}=4$ theories. In this way, we will be able to clearly identify whether emerging integrability phenomena are sensitive to the representation in which the fermions fields are defined as well as to the presence of scalars and, on top of it, supersymmetry.

Our analysis will be entirely formulated in the momentum space, i.e., we will calculate the two-loop kernel $\mathbb{V}$ of the evolution equation (3.18). It is straightforward to translate the obtained expressions from the momentum to the configuration space by applying (3.20). As we will argue, to two-loop order, the three-fermion operators (2.19) and (2.20) evolve autonomously and the perturbative expansion of the evolution kernel reads in the momentum representation

$$
\mathbb{V}(\boldsymbol{u} \mid \boldsymbol{v})=\lambda \mathbb{V}^{(0)}(\boldsymbol{u} \mid \boldsymbol{v})+\lambda^{2} \mathbb{V}^{(1)}(\boldsymbol{u} \mid \boldsymbol{v})+\mathcal{O}\left(\lambda^{3}\right)
$$

where depending on the representation of the fermion fields, the coupling constant $\lambda$ is given by the corresponding expression in (4.10). The one-loop result was given earlier in Eq. (4.13). In addition, to simplify the calculation we shall apply the Brodsky-Lepage limit and assume that the $v$ - and $u$-variables take nonnegative values and belong to the simplices (4.16) and (4.18), respectively. This does not lead to a loss of generality since the resulting expression for $\mathbb{V}(\boldsymbol{u} \mid \boldsymbol{v})$ can be uniquely continued to arbitrary values of the scaling variables. As before, the calculation will be performed in the light-like axial gauge $A_{+}(x)=0$ within the $\overline{\mathrm{DR}}-$ and $\overline{\mathrm{MS}}$-renormalization schemes.

The two-loop Feynman diagrams contributing to $\mathbb{V}^{(1)}$ in the light-cone gauge can be separated into three different sets sorted according to the number of fermions involved in the interaction, i.e., one-, two- and three-particle irreducible contributions. Their contribution to the evolution kernel can be written as

$$
\mathbb{V}^{(1)}(\boldsymbol{u} \mid \boldsymbol{v})=\mathbb{V}_{123}^{(1)}+\mathbb{V}_{231}^{(1)}+\mathbb{V}_{312}^{(1)}+\mathbb{V}_{12}^{(1)}+\mathbb{V}_{23}^{(1)}+\mathbb{V}_{31}^{(1)}+\mathbb{V}_{1}^{(1)}+\mathbb{V}_{2}^{(1)}+\mathbb{V}_{3}^{(1)}
$$

where the additional terms have been added to ensure the invariance of the evolution kernel under cyclic permutations of quarks. Denoting the kernel of the operators $\mathbb{V}_{123}^{(1)}$ and $\mathbb{V}_{12}^{(1)}$ as $\mathbb{V}^{(1)}\left(u_{1}, u_{2}, u_{3} \mid v_{1}, v_{2}, v_{3}\right)$ and $\mathbb{V}^{(1)}\left(u_{1}, u_{2} \mid v_{1}, v_{2}\right)$, respectively, one has

$$
\mathbb{V}_{i j k}^{(1)}=\mathbb{V}^{(1)}\left(u_{i}, u_{j}, u_{k} \mid v_{i}, v_{j}, v_{k}\right), \quad \mathbb{V}_{j k}^{(1)}=\mathbb{V}^{(1)}\left(u_{j}, u_{k} \mid v_{j}, v_{k}\right)
$$

The three-particle irreducible kernel $\mathbb{V}_{123}^{(1)}$ receives contributions from the diagrams shown in Fig. 2. The set of two-loop diagrams contributing to the two-particle kernel $\mathbb{V}_{12}^{(1)}$ is displayed in Figs. 4 and 6. Among these diagrams one can distinguish those without scalars (see Fig. 4). They are exactly the same as involved in renormalization of twist-two maximal-helicity operators in QCD [37, 38. However, as we will see momentarily, the important difference with the twisttwo operators is that for three-fermion operators (2.19) and (2.20) each pair of particles has a nonvanishing color charge and this affects the color factors accompanying individual diagrams. Finally, the self-energy corrections to gaugino/quark fields induce c-number corrections $\mathbb{V}_{i}^{(1)}$, see Fig. [7, which have the structure

$$
\mathbb{V}_{i}^{(1)}=\xi_{i}^{(1)} \delta\left(u_{1}-v_{1}\right) \delta\left(u_{2}-v_{2}\right)
$$

with $\xi_{i}^{(1)}$ being the residue of the renormalization constant of $i^{\text {th }}$ fermion line to two loop order.

Let us consider three different contributions to $\mathbb{V}^{(1)}(\boldsymbol{u} \mid \boldsymbol{v})$ one after another. 


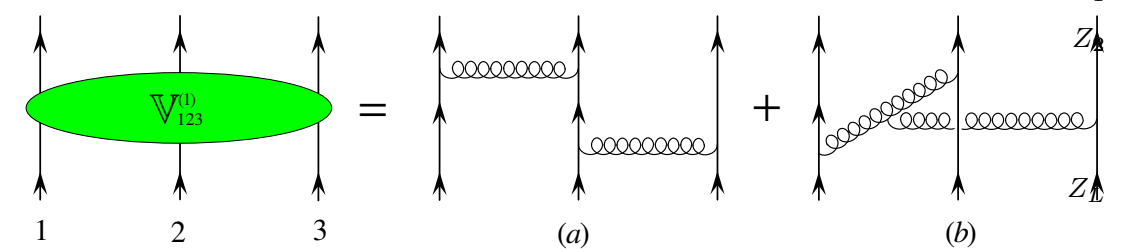

Figure 2: The three-particle contributions (with mirror symmetrical graphs tacitly implied) to the three-particle dilatation operator.

\subsection{Three-particle contributions}

The three-particle irreducible kernel $\mathbb{V}_{123}^{(1)}$ receives contribution from Feynman diagrams which either represent an iteration of a single-particle exchange, or contain a triple gluon vertex (see Figs. 2 (a) and (b), respectively). In QCD and $\mathcal{N}=1$ SYM theory, such kind of diagrams can only involve gluon exchanges while in the $\mathcal{N}=2$ and $\mathcal{N}=4$ theories scalars may, in principle, propagate instead of gluons in internal lines. As it was explained in Sect. 4.2. by virtue of the maximal $R$-charge of the three-particle operator (2.19), the scalar fields do not contribute to the two-particle kernel $\mathbb{H}_{12}^{(0)}$ to one loop order. The very same argument applies to the three-particle irreducible kernel $\mathbb{V}_{123}^{(1)}$. In other words, the diagrams with scalar exchanges vanish identically and the evolution kernel $\mathbb{V}_{123}^{(1)}$ takes the same form in SYM theories with $\mathcal{N}=1,2,4$.

Another interesting feature of the kernel $\mathbb{V}_{123}^{(1)}$ is that the diagram in Fig. 2 (b) involving the three-gluon vertex vanishes identically. This property holds true independently on the representation in which the fermion fields are defined and, therefore, it is valid both in QCD and SYM theories. Namely, for the quarks in the fundamental representation of the $S U(3)$ the color factor $C$ corresponding to the diagram in Fig. [2 (b) equals

$$
\varepsilon^{i_{1} i_{2} i_{3}} f^{a b c}\left(t^{a}\right)_{i_{1} j_{1}}\left(t^{b}\right)_{i_{2} j_{2}}\left(t^{c}\right)_{i_{3} j_{3}}=C \varepsilon^{j_{1} j_{2} j_{3}} .
$$

The two sides of this relation have an opposite parity under permutations $j_{1} \rightleftarrows j_{2}$ leading to $C=0$. In the similar manner, for the gaugino in the adjoint representation of the $S U\left(N_{c}\right)$ the color factor equals

$$
f^{a b c} T_{1}^{a} T_{2}^{b} T_{3}^{c}=-f^{a b c} T_{1}^{a} T_{2}^{b}\left(T_{1}^{c}+T_{2}^{c}\right)=0,
$$

where $\left(T_{j}^{a}\right)_{b c}=i f^{a b c}$ is the color charge of $j^{\text {th }}$ particle and $f^{a b c}=-f^{a c b}$ are the $S U\left(N_{c}\right)$ structure constants. Here in the first relation we used the color neutrality of the single-trace three-particle operator, i.e., $\sum_{j=1,2,3} T_{j}^{a}=0$, and applied the identity $f^{a b c} T_{j}^{a} T_{j}^{b}=-\frac{i}{2} N_{c} T_{j}^{c}$. In arriving at (5.6), it was crucial that the color-singlet operator (2.19) is built from $L=3$ particles. For $L>3$, the contribution of the diagram in Fig. 2 (b) to the multi-particle single-trace operator is different from zero but it is suppressed in the multi-color limit as $\sim 1 / N_{c}^{2}$ since the diagram is nonplanar.

We conclude that the three-particle irreducible kernel $\mathbb{V}_{123}^{(1)}$ is determined by the Feynman diagram shown in Fig. 2 (a) and its mirror image. The two diagrams describe iteration of onegluon exchange and the corresponding color factor is merely the square of the one-loop color factor. For gluinos in the adjoint representation it yields $T_{1}^{a} T_{2}^{a} T_{2}^{b} T_{3}^{b}=\left(-\frac{N_{c}}{2}\right)^{2}$, where we have used the identity (4.8) twice. For fundamental quarks, one uses in the same fashion Eq. (4.9). In both cases the color factors can be absorbed into the redefinition of the coupling constants (4.10) and, as a result, $\mathbb{V}_{123}^{(1)}$ has the same form in QCD and in all SYM theories. 
The evolution kernel $\mathbb{V}_{123}^{(1)}(\boldsymbol{u} \mid \boldsymbol{v})$ depends on two sets of momenta. As was already mentioned, we restrict our analysis to the values of variables belonging to the simplices (4.16) and (4.18). For given values of the $v$-variables and $u_{1}+u_{2}+u_{3}=1$, the three-particle irreducible kernel $\mathbb{V}_{123}^{(1)}(\boldsymbol{u} \mid \boldsymbol{v})$ has support inside the triangle $0 \leq u_{1}, u_{3} \leq 1$ and $u_{1}+u_{3} \leq 1$ shown in Fig. 3. Three sides of the triangle corresponds to $u_{1}=0, u_{2}=0$ and $u_{3}=0$. Due to a larger variety of possibilities of momentum redistributions between three interacting fields, $\mathbb{V}_{123}^{(1)}(\boldsymbol{u} \mid \boldsymbol{v})$ has a more complex structure as compared with the two-particle kernel (4.17). A simple analysis demonstrates that there exist six distinct regions in the phase space displayed in Fig. 3. the diagram in Fig. 2 (a) contains two internal gluon lines carrying the light-cone momenta $v_{1}-u_{1}$ and $u_{3}-v_{3}$ and the virtual fermion line with the momentum $v_{1}+v_{2}-u_{1}$. For the mirror symmetric diagram the latter momentum reads $v_{3}+v_{2}-u_{3}$. In distinction with the external $u$-variables, the momenta flowing through internal lines are not necessarily positive in the Brodsky-Lepage limit. For $v_{1}>u_{1}$ we can readily identify two regions: region 3 for $v_{3}>u_{3}$ and combined regions 1 and 2 for $v_{3}<u_{3}$. In these kinematical regions the internal fermionic line does not bring in any new restrictions since the momentum flow is always positive $v_{1}+v_{2}-u_{1}>0$. Considering now the situation of the negative momentum flow in the first gluon line, i.e., $v_{1}<u_{1}$, one immediately finds that the virtual quark/gluino line can have either positive $\left(v_{1}+v_{2}>u_{1}\right)$ or negative $\left(v_{1}+v_{2}<u_{2}\right)$ momentum flow, which is laid over the restriction from the second gluon line $v_{3} \gtrless u_{3}$. From these we find three regions in the phase space labelled as 4, 5 and 6 . Notice that the boundary $v_{1}+v_{2}=u_{1}$ is reflected from the boundary $u_{2}=0$ of the support region into the boundary $u_{3}=v_{3}$. The consideration of the mirror symmetric diagram to Fig. 2 (a) allows one to further separate the regions 1 and 2 from the positivity/negativity of the momentum flow of the internal fermion line. The above analysis covers all regions in the support region of the two-loop three-particle irreducible kernel.

Summarizing, one decomposes the three-particle irreducible kernel as follows

$$
\begin{aligned}
\mathbb{V}_{123}^{(1)}(\boldsymbol{u} \mid \boldsymbol{v}) & =\theta\left(u_{3}-\bar{v}_{1}\right) \mathbb{F}_{1}(\boldsymbol{u} \mid \boldsymbol{v})+\theta\left(\bar{v}_{1}-u_{3}\right) \theta\left(v_{1}-u_{1}\right) \theta\left(u_{3}-v_{3}\right) \mathbb{F}_{2}(\boldsymbol{u} \mid \boldsymbol{v}) \\
& +\theta\left(v_{1}-u_{1}\right) \theta\left(v_{3}-u_{3}\right) \mathbb{F}_{3}(\boldsymbol{u} \mid \boldsymbol{v})+\theta\left(u_{1}-v_{1}\right) \theta\left(u_{3}-v_{3}\right) \mathbb{F}_{4}(\boldsymbol{u} \mid \boldsymbol{v}) \\
& +\theta\left(v_{3}-\bar{u}_{1}\right) \mathbb{F}_{5}(\boldsymbol{u} \mid \boldsymbol{v})+\theta\left(\bar{u}_{1}-v_{3}\right) \theta\left(u_{1}-v_{1}\right) \theta\left(v_{3}-u_{3}\right) \mathbb{F}_{6}(\boldsymbol{u} \mid \boldsymbol{v})
\end{aligned}
$$

where $\bar{u}_{j}=1-u_{j}, \bar{v}_{j}=1-v_{j}$ and the function $\mathbb{F}_{i}(\boldsymbol{u} \mid \boldsymbol{v})$ denotes the contribution of the $i^{\text {th }}$ region on the phase diagram in Fig. 3. It is tacitly assumed that the $u$ - and $v$-momenta belong to the simplices (4.18) and (4.16), respectively. The sum of the diagrams shown in Fig. 22 is symmetric under the interchange of the $1^{\text {st }}$ and $3^{\text {rd }}$ lines. Therefore the kernel (5.7) has to be invariant under the interchange of the corresponding momenta $\left\{u_{1}, u_{3} \mid v_{1}, v_{3}\right\} \leftrightarrow\left\{u_{3}, u_{1} \mid v_{3}, v_{1}\right\}$, which yields the following relations

$$
\begin{aligned}
& \mathbb{F}_{3}\left(u_{1}, u_{2}, u_{3} \mid v_{1}, v_{2}, v_{3}\right)=\mathbb{F}_{3}\left(u_{3}, u_{2}, u_{1} \mid v_{3}, v_{2}, v_{1}\right), \\
& \mathbb{F}_{4}\left(u_{1}, u_{2}, u_{3} \mid v_{1}, v_{2}, v_{3}\right)=\mathbb{F}_{4}\left(u_{3}, u_{2}, u_{1} \mid v_{3}, v_{2}, v_{1}\right), \\
& \mathbb{F}_{5}\left(u_{1}, u_{2}, u_{3} \mid v_{1}, v_{2}, v_{3}\right)=\mathbb{F}_{2}\left(u_{3}, u_{2}, u_{1} \mid v_{3}, v_{2}, v_{1}\right), \\
& \mathbb{F}_{6}\left(u_{1}, u_{2}, u_{3} \mid v_{1}, v_{2}, v_{3}\right)=\mathbb{F}_{1}\left(u_{3}, u_{2}, u_{1} \mid v_{3}, v_{2}, v_{1}\right),
\end{aligned}
$$

so that the three-particle kernel (5.7) only involves four nontrivial functions. Moreover, as we will see in a moment, one of these functions vanishes.

To determine the functions $\mathbb{F}_{i}$ one has to evaluate the three-particle connected diagrams displayed in Fig. 2 using particular renormalization scheme to treat ultraviolet divergences and 


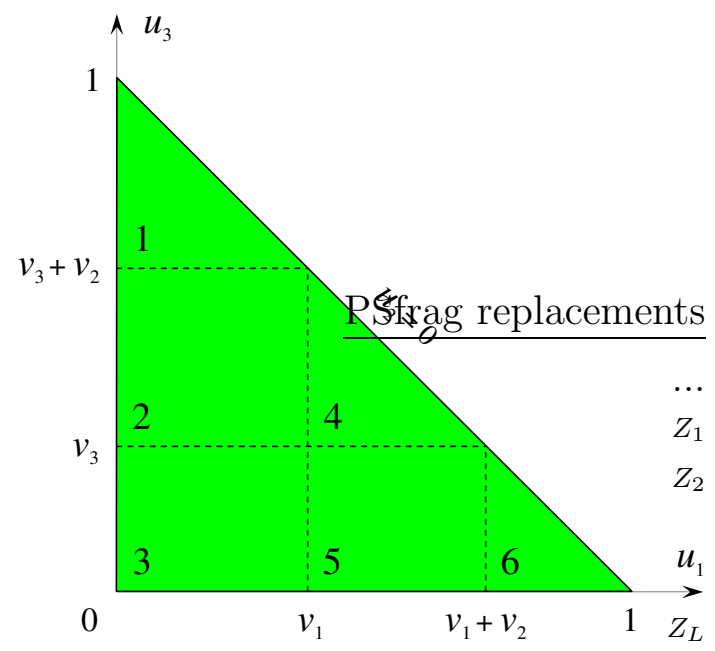

Figure 3: Phase-space regions of three-particle irreducible kernel.

extract $\mathbb{V}_{123}^{(1)}(\boldsymbol{u} \mid \boldsymbol{v})$ from a single pole $\sim 1 / \varepsilon$ contribution using (4.5). The calculation can be straightforwardly performed either in the covariant or light-cone formalism. We found that the contribution of diagrams in Fig. 2 to $\mathbb{V}_{123}^{(1)}(\boldsymbol{u} \mid \boldsymbol{v})$ is the same in the $\overline{\mathrm{DR}}-$ and $\overline{\mathrm{MS}}$-schemes. It leads to the following expressions for the $\mathbb{F}$-functions

$$
\begin{aligned}
\mathbb{F}_{1} & =\frac{1}{2} f\left(u_{1}, v_{1}\right) f\left(\bar{v}_{1}-u_{3}, v_{2}\right) \ln \frac{\left(u_{1}-v_{1}\right)\left(u_{3}-v_{3}\right)}{\left(\bar{v}_{1}-u_{3}\right)\left(\bar{u}_{1}-v_{3}\right)} \\
& +\frac{1}{2} f\left(u_{1}, v_{1}\right) f\left(u_{2}, \bar{u}_{1}-v_{3}\right) \ln \frac{u_{1}\left(\bar{u}_{1}-v_{3}\right)^{2}}{u_{2} \bar{v}_{3}\left(u_{3}-v_{3}\right)}+\frac{1}{2} f\left(u_{2}, \bar{v}_{1}-u_{3}\right) f\left(\bar{v}_{1}-u_{3}, v_{2}\right) \ln \frac{v_{1}\left(\bar{u}_{1}-v_{3}\right)}{\bar{v}_{3}\left(v_{1}-u_{1}\right)}, \\
\mathbb{F}_{2} & =\frac{1}{2} f\left(u_{1}, v_{1}\right) f\left(\bar{v}_{1}-u_{3}, v_{2}\right) \ln \frac{\bar{u}_{3}\left(u_{3}-v_{3}\right)}{u_{1}\left(\bar{u}_{1}-v_{3}\right)} \\
& +\frac{1}{2} f\left(u_{1}, v_{1}\right) f\left(u_{2}, \bar{u}_{1}-v_{3}\right) \ln \frac{u_{1}\left(\bar{u}_{1}-v_{3}\right)^{2}}{u_{2} \bar{v}_{3}\left(u_{3}-v_{3}\right)}+\frac{1}{2} f\left(u_{2}, \bar{u}_{1}-v_{3}\right) f\left(\bar{u}_{1}-v_{3}, v_{2}\right) \ln \frac{\bar{u}_{3}\left(\bar{u}_{1}-v_{3}\right)}{u_{2} \bar{v}_{3}}, \\
\mathbb{F}_{3} & =0 \\
\mathbb{F}_{4} & =\frac{1}{2} f\left(u_{2}, \bar{u}_{1}-v_{3}\right) f\left(\bar{u}_{1}-v_{3}, v_{2}\right) \ln \frac{\bar{u}_{1} \bar{u}_{3}\left(\bar{v}_{1}-u_{3}\right)\left(\bar{u}_{1}-v_{3}\right)}{u_{2}^{2} \bar{v}_{1} \bar{v}_{3}} \\
& +\frac{1}{2} f\left(u_{1}, v_{1}\right) f\left(u_{2}, \bar{u}_{1}-v_{3}\right) \ln \frac{u_{1} v_{2}}{\bar{v}_{3}\left(u_{1}-v_{1}\right)}+\frac{1}{2} f\left(u_{1}, v_{1}\right) f\left(\bar{v}_{1}-u_{3}, v_{2}\right) \ln \frac{\bar{u}_{3}\left(u_{1}-v_{1}\right)}{u_{1}\left(\bar{v}_{1}-u_{3}\right)} \\
& +\frac{1}{2} f\left(u_{2}, \bar{v}_{1}-u_{3}\right) f\left(u_{3}, v_{3}\right) \ln \frac{u_{3} v_{2}}{\bar{v}_{1}\left(u_{3}-v_{3}\right)}+\frac{1}{2} f\left(\bar{u}_{1}-v_{3}, v_{2}\right) f\left(u_{3}, v_{3}\right) \ln \frac{\bar{u}_{1}\left(u_{3}-v_{3}\right)}{u_{3}\left(\bar{u}_{1}-v_{3}\right)}
\end{aligned}
$$

with $\bar{v}_{i}=1-v_{i}$ and $\bar{u}_{i}=1-u_{i}$. The remaining functions can be deduced making use of the symmetry relations (5.8). Here the notation was introduced for the function

$$
f(u, v)=\frac{u}{v} \frac{1}{v-u},
$$

which we already encountered at one loop, see Eq. (4.17).

A few comments are in order. According to (5.12), the third region in Fig. 3 produces a vanishing contribution to the three-particle kernel. This comes about as a result of cancellation 
of the contribution of the diagram shown in Fig. 2 (a) against the one coming from a mirror symmetric diagram.

It is known from the QCD calculations that, in general, two-loop evolution kernels involve special functions (dilogarithms $\operatorname{Li}_{2}(x)$ ). Still, the two-loop result for $\mathbb{V}_{123}^{(1)}(\boldsymbol{u} \mid \boldsymbol{v})$ is represented in terms of elementary functions only: the $\mathbb{F}$-functions are given by a product of one-loop elements (5.14) dressed by logarithmic dependence on the light-cone momenta.

The function $f(u, v)$ has a pole at $u=v$. In the expression for the one-loop evolution kernel, Eq. (4.17), this pole is regularized by the '+'-prescription. To two loops, the $\mathbb{F}$-functions involve the product of two $f$-functions and develop poles at $u_{1}=v_{1}$ and $u_{3}=v_{3}$. It turns out that these poles cancel in the sum (5.7) in such a way that the evolution kernel $\mathbb{V}_{123}^{(1)}(\boldsymbol{u} \mid \boldsymbol{v})$ only has integrable singularities at $u_{1}=v_{1}$ and $u_{3}=v_{3}$. It is easy to see that the two singularities correspond to the limit when the internal gluons in the diagram shown in Fig. 2 (a) have vanishing light-cone momenta, $k_{+}=0$. We remind that, in the light-cone axial gauge $A_{+}=0$, the gauge field propagator (4.1) is not well-defined for $k_{+}=0$ and has to be supplemented by a particular prescription, Eqs. (4.2) and (4.3). The fact that the two-loop kernel (5.7) is integrable at $u_{1}=v_{1}$ and $u_{3}=v_{3}$ implies that $\mathbb{V}_{123}^{(1)}(\boldsymbol{u} \mid \boldsymbol{v})$ is not sensitive to the choice of the axial gauge prescription and, most importantly, it has finite moments $\int[d u]_{3} \mathbb{V}_{123}^{(1)}(\boldsymbol{u} \mid \boldsymbol{v}) u_{1}^{k_{1}} u_{2}^{k_{2}} u_{3}^{k_{3}}$.

We remind that the entire two-loop kernel (5.2) has to fulfill the polynomiality condition, that is, its moments ought to be polynomial in the $v$-variables, Eq. (3.22). It turns out that the threeparticle kernel $\mathbb{V}_{123}^{(1)}(\boldsymbol{u} \mid \boldsymbol{v})$ alone does not obey this condition. Its moments are given by rational functions of $v_{1,2,3}$ decorated by logarithms and dilogarithms depending on the ratio $v_{1} / v_{3}$ and $v_{2} / v_{3}$. As we will demonstrate in Sect. 5.2.3, the "unwanted" terms violating the polynomiality of $\mathbb{V}_{123}^{(1)}(\boldsymbol{u} \mid \boldsymbol{v})$ cancel in the right-hand side of Eq. (15.2) against similar terms coming from the moments of two-particle irreducible kernels $\mathbb{V}_{j k}^{(1)}$.

To separate the "unwanted" terms it proves convenient to introduce the double plus-distribution. It represents a natural generalization of (4.15) for three-particle irreducible kernels and is defined as

$$
\begin{aligned}
\int_{0}^{1}[d u]_{3} \varphi & \left(u_{1}, u_{3}\right)\left[\mathbb{V}_{123}^{(1)}(\boldsymbol{u} \mid \boldsymbol{v})\right]_{++} \\
& =\int_{0}^{1}[d u]_{3}\left[\varphi\left(u_{1}, u_{3}\right)-\varphi\left(v_{1}, u_{3}\right)-\varphi\left(u_{1}, v_{3}\right)+\varphi\left(v_{1}, v_{3}\right)\right] \mathbb{V}_{123}^{(1)}(\boldsymbol{u} \mid \boldsymbol{v})
\end{aligned}
$$

where $\varphi\left(u_{1}, u_{3}\right)$ is a test function. Since the kernel $\mathbb{V}_{123}^{(1)}(\boldsymbol{u} \mid \boldsymbol{v})$ lives on the simplices (4.16) and (4.18), as exhibited by the step-function structure, the constraint on the $u$-momentum fractions arising from the integration measure can be omitted, i.e., $\theta\left(1-u_{1}-u_{3}\right) \mathbb{V}_{123}^{(1)}(\boldsymbol{u} \mid \boldsymbol{v})=\mathbb{V}_{123}^{(1)}(\boldsymbol{u} \mid \boldsymbol{v})$. One can verify then that the kernel $\left[\mathbb{V}_{123}^{(1)}(\boldsymbol{u} \mid \boldsymbol{v})\right]_{++}$defined in this manner satisfies the polynomiality condition. By definition,

$$
\begin{aligned}
\mathbb{V}_{123}^{(1)}(\boldsymbol{u} \mid \boldsymbol{v}) & =\left[\mathbb{V}_{123}^{(1)}(\boldsymbol{u} \mid \boldsymbol{v})\right]_{++}+\delta\left(u_{1}-v_{1}\right) \int_{0}^{1} d u_{1} \mathbb{V}_{123}^{(1)}(\boldsymbol{u} \mid \boldsymbol{v}) \\
& +\delta\left(u_{3}-v_{3}\right) \int_{0}^{1} d u_{3} \mathbb{V}_{123}^{(1)}(\boldsymbol{u} \mid \boldsymbol{v})-\delta\left(u_{1}-v_{1}\right) \delta\left(u_{3}-v_{3}\right) \int_{0}^{1}[d u]_{3} \mathbb{V}_{123}^{(1)}(\boldsymbol{u} \mid \boldsymbol{v})
\end{aligned}
$$

where the integrals entering the subtraction terms are well-defined and can be evaluated with the help of (5.7) (see Eqs. (B.3)-(B.5) in Appendix B $)$. We conclude that the polynomiality 


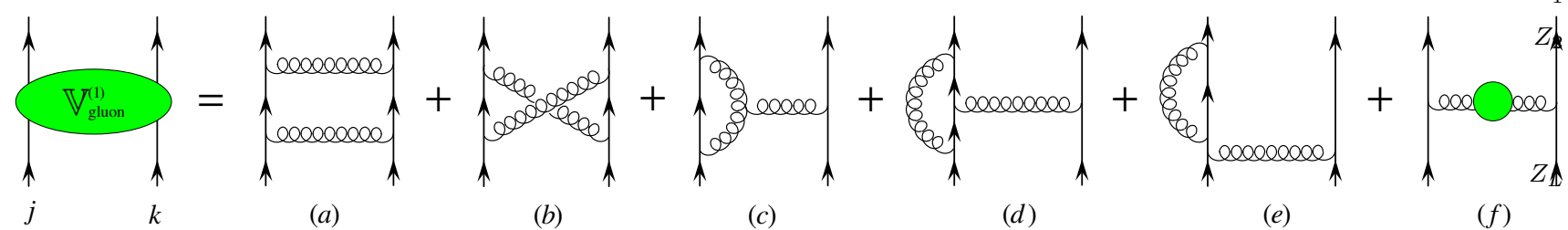

Figure 4: Two-loop corrections to the two-particle evolution kernel involving only gluons in internal lines. The mirror symmetrical diagrams are not displayed. The gluon polarization

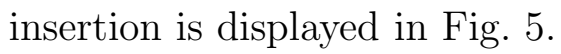

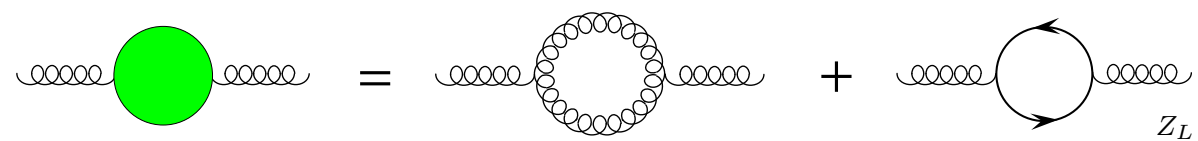

Figure 5: One-loop gluon polarization contributing as an insertion into the one-loop gluon exchange diagram. The gluon and fermion bubbles in Fig. [2 (f) are be denoted as (f) $)_{g l}$ and $(f)_{\text {fer }}$, respectively.

is violated by the subtraction terms in the right-hand side of (5.16). The first two subtraction terms preserve the momenta of a single particle and take the form of two-particle irreducible kernels. The last subtraction term has the structure of a single-particle contribution with the only difference that it involves the integral $\int[d u]_{3} \mathbb{V}_{123}^{(1)}(\boldsymbol{u} \mid \boldsymbol{v})$ which is a rather complicated function of all three momentum fractions, $v_{1,2,3}$. Later in this section, we will demonstrate that the subtraction terms are compensated by similar polynomiality breaking terms coming from genuine two-particle evolution kernels.

\subsection{Two-particle contributions}

Let us now consider the two-particle irreducible contribution to the two-loop evolution kernel (5.2). It is determined by Feynman diagrams shown in Figs. 4 and 6 . In distinction with the three-particle kernel, the two-particle kernel $\mathbb{V}_{12}^{(1)}$ is not universal and depends on the gauge theory under consideration. For the sake of simplicity we will distinguish between QCD-like diagrams, that is, those involving only gluons, and diagrams with scalars. The former are relevant for both QCD and SYM theories whereas the latter only appear in $\mathcal{N}=2$ and $\mathcal{N}=4$ theories. Let us analyze the two sets of diagrams in turn.

\subsubsection{Gluon contribution}

The diagrams with gluon exchanges are shown in Fig. 4. As was already mentioned, similar diagrams define the two-loop contribution to the evolution kernel for the twist-two maximal helicity operators in QCD defined in (2.18). The important difference between the three-particle operators, Eqs. (2.20) and (2.19), and the two-particle operators, Eqs. (2.18) and (2.17), is that in the latter case the pair of fermions is in the color-singlet state. However, this only affects the color factors accompanying the individual Feynman diagrams shown in Fig. 团 not their momentum 
structure. Let us examine these color factors and relate to each other the two-particle contribution to the dilatation operators for the two- and three-particle operators.

To start with, we present the result for the two-loop evolution kernel for the twist-two operators (2.18) in QCD. In the momentum representation, it has the following form for $v_{1}+v_{2}=$ $u_{1}+u_{2}=1$

$$
\mathbb{V}_{\mathrm{QCD}}\left(u_{1}, u_{2} \mid v_{1}, v_{2}\right)=\frac{g^{2} C_{F}}{4 \pi^{2}} \mathbb{V}_{\mathrm{QCD}}^{(0)}\left(u_{1}, v_{1}\right)+\left(\frac{g^{2} C_{F}}{4 \pi^{2}}\right)^{2} \mathbb{V}_{\mathrm{QCD}}^{(1)}\left(u_{1}, v_{1}\right)
$$

where $C_{F}=\left(N_{c}^{2}-1\right) /\left(2 N_{c}\right)$ is the Casimir operator in the fundamental representation of the $S U\left(N_{c}\right)$. To one-loop order, the evolution kernel is determined by the universal expression (4.17)

$$
\mathbb{V}_{\mathrm{QCD}}^{(0)}(u, v)=\frac{u}{v} \frac{\theta(v-u)}{v-u}+\left\{\begin{array}{l}
u \rightarrow \bar{u} \\
v \rightarrow \bar{v}
\end{array}\right\}
$$

To two-loop order, the kernel $\mathbb{V}_{\mathrm{QCD}}^{(1)}(u, v)$ has been first calculated in Ref. [37] in the so-called forward limit, for $v_{1}+v_{2}=u_{1}+u_{2}=0$. It was later reconstructed for $u_{1}+u_{2} \neq 0$, making use of the conformal symmetry constraints, and yielded the result 38.

$$
\mathbb{V}_{\mathrm{QCD}}^{(1)}(u, v)=\mathbb{V}_{F}^{(1)}(u, v)+\frac{C_{F}-\frac{1}{2} N_{c}}{C_{F}} \mathbb{V}_{A}^{(1)}(u, v)+\frac{\beta_{\mathrm{QCD}, 0}}{C_{F}} \mathbb{V}_{\beta}^{(1)}(u, v)+\left\{\begin{array}{l}
u \rightarrow \bar{u} \\
v \rightarrow \bar{v}
\end{array}\right\}
$$

where $\beta_{\mathrm{QCD}, 0}=11 N_{c} / 3-2 n_{f} / 3$ is the one-loop beta-function in QCD, Eq. (2.8), and

$$
\begin{aligned}
\mathbb{V}_{F}^{(1)}(u, v) & =\frac{1}{2 v} \ln \bar{u} \ln u \\
& +\theta(v-u)\left[f(u, v)\left(\frac{2}{3}-\zeta(2)-\frac{3}{4} \ln \frac{u}{v}-\frac{1}{2} \ln \frac{u}{v} \ln \frac{v-u}{u}\right)+\frac{1}{2} f(\bar{u}, \bar{v}) \ln \frac{u}{v} \ln \frac{v-u}{v}\right] \\
\mathbb{V}_{A}^{(1)}(u, v) & =\theta(v-u)\left[f(u, v)\left(-\frac{2}{3}-\operatorname{Li}_{2}(u)-\operatorname{Li}_{2}(\bar{v})\right)+f(\bar{u}, \bar{v}) \ln \bar{u} \ln v+\frac{u}{2 v}\right] \\
& -\theta(v-\bar{u})\left[f(u, v)\left(\operatorname{Li}_{2}\left(1-\frac{u}{v}\right)+\operatorname{Li}_{2}(\bar{v})+\frac{1}{2} \ln ^{2} v-\ln u \ln v\right)\right. \\
& \left.+f(\bar{u}, \bar{v})\left(\operatorname{Li}_{2}(\bar{u})-\operatorname{Li}_{2}\left(1-\frac{u}{v}\right)-\frac{1}{2} \ln ^{2} v\right)-\frac{\bar{u}}{2 v}\right], \\
\mathbb{V}_{\beta}^{(1)}(u, v) & =\theta(v-u)\left[\frac{1}{4} \ln \frac{u}{v}+\frac{5}{12}\right] f(u, v) .
\end{aligned}
$$

Here $\zeta(n)$ is the Riemann zeta function, $\operatorname{Li}_{2}(x)=-\int_{0}^{x} \frac{d t}{t} \ln (1-t)$ is the Euler dilogarithm and the $f$-function is defined in (5.14).

Comparing the QCD expression (5.17) with Eqs. (3.2), (4.10) and (4.17), we see that to one-loop order the evolution kernel $\mathbb{V}_{12}$ for three-particle operators can be deduced from (5.17) through substitution of the color factors

$$
C_{F} \rightarrow \frac{2}{3}, \quad C_{F} \rightarrow \frac{N_{c}}{2}
$$

for fermions in the fundamental and adjoint representations, respectively. Unfortunately, this simple property gets lost starting from two loops. To see this, one examines the color factors 
corresponding to the Feynman diagrams shown in Fig. 4 in four different cases: twist-two operator (2.18) in QCD, its counter-part (2.17) in SYM theory, three-quark operator (2.20) in QCD and three-gaugino (2.19) operator in SYM.

\begin{tabular}{||c|c|c|c|c|c|c|c||}
\hline \hline $\mathbb{O}$ & $(\mathrm{a})$ & $(\mathrm{b})$ & $(\mathrm{c})$ & $(\mathrm{d})$ & $(\mathrm{e})$ & $(\mathrm{f})_{\mathrm{gl}}$ & $(\mathrm{f})_{\mathrm{fer}}$ \\
\hline \hline $\bar{q}_{i} q^{i}$ & $C_{F}^{2}$ & $C_{F}\left(C_{F}-\frac{1}{2} N_{c}\right)$ & $\frac{1}{2} C_{F} N_{c}$ & $C_{F}\left(C_{F}-\frac{1}{2} N_{c}\right)$ & $C_{F}^{2}$ & $\frac{1}{2} C_{F} N_{c}$ & $\frac{1}{2} C_{F} n_{f}$ \\
\hline$\varepsilon_{i j k} q^{i} q^{j} q^{k}$ & $\frac{4}{9}$ & $-\frac{5}{9}$ & 1 & $-\frac{1}{9}$ & $\frac{8}{9}$ & 1 & $\frac{1}{3} n_{f}$ \\
\hline \hline $\operatorname{tr}\{\lambda \lambda\}$ & $N_{c}^{2}$ & $\frac{1}{2} N_{c}^{2}$ & $\frac{1}{2} N_{c}^{2}$ & $\frac{1}{2} N_{c}^{2}$ & $N_{c}^{2}$ & $\frac{1}{2} N_{c}^{2}$ & $\frac{1}{2} N_{c}^{2} \mathcal{N}$ \\
\hline $\operatorname{tr}\{\lambda \lambda \lambda\}$ & $\frac{1}{4} N_{c}^{2}$ & 0 & $\frac{1}{4} N_{c}^{2}$ & $\frac{1}{4} N_{c}^{2}$ & $\frac{1}{2} N_{c}^{2}$ & $\frac{1}{4} N_{c}^{2}$ & $\frac{1}{4} N_{c}^{2} \mathcal{N}$ \\
\hline \hline
\end{tabular}

We notice that for two-particle operators, going over from QCD to SYM amounts to replacing $C_{F}$ by the Casimir operator in the adjoint representation $C_{F} \rightarrow N_{c}$ and substituting the number of quark flavors as $n_{f} \rightarrow N_{c} \mathcal{N}$ (see Eq. (2.6) ). Let us now turn to three-particle operators and substitute the color factors in the second row of the table according to (5.23) (we recall that the three-quark operators are well-defined for $N_{c}=3$ only). It is easy to see that this leads to the correct expressions for the color factors in the third and fifth rows except for the columns (d) and (e). Thus, in order to reconstruct the two-particle contribution to the evolution kernels for the three-quark and three-gaugino operators from the QCD expression (5.19) with the help of (5.23), it suffices to supplement (5.19) with the contribution of two additional diagrams in QCD shown in Fig. 4 (d) and (e). More precisely, one only needs the abelian part of their contribution, i.e., the one proportional to $C_{F}^{2}$. In what follows, we denote the corresponding kernel as $\mathbb{V}_{\mathrm{SV}}^{(1)}$. A straightforward calculation leads to

$$
\begin{aligned}
\mathbb{V}_{\mathrm{SV}}^{(1)}(u, v)=\theta(v-u)\left[f ( u , v ) \left(-\frac{3}{4} \ln \frac{u}{v}-\frac{1}{4} \ln ^{2} \frac{u}{v}-\frac{1}{2} \ln \frac{u}{v} \ln \frac{(v-u) \bar{u}}{v \bar{v}}\right.\right. \\
\left.\quad+\frac{1}{2} \operatorname{Li}_{2}\left(\frac{u-v}{\bar{v}}\right)-\frac{1}{2} \operatorname{Li}_{2}\left(\frac{v-u}{v}\right)+\frac{1}{2} \operatorname{Li}_{2}(u)-\frac{1}{2} \operatorname{Li}_{2}(v)-\frac{1}{2} \zeta(2)\right) \\
+f(\bar{u}, \bar{v})\left(\frac{1}{2} \ln \frac{v-u}{v} \ln \frac{u}{v}-\frac{1}{4} \ln ^{2} \frac{\bar{u}}{\bar{v}}-\frac{1}{2} \ln \bar{u} \ln v\right. \\
\left.\left.\quad+\frac{1}{2} \operatorname{Li}_{2}(u)-\frac{1}{2} \operatorname{Li}_{2}(v)+\frac{1}{2} \operatorname{Li}_{2}\left(\frac{v-u}{v}\right)-\frac{1}{2} \operatorname{Li}_{2}\left(\frac{u-v}{\bar{v}}\right)-\frac{1}{2} \zeta(2)\right)\right] \\
+\left\{\begin{array}{l}
u \rightarrow \bar{u} \\
v \rightarrow \bar{v}
\end{array}\right\} .
\end{aligned}
$$

We recall that (5.17) is defined for $v_{1}+v_{2}=u_{1}+u_{2}=1$ while for three-particle operator the twoparticle evolution kernel, say $\mathbb{V}_{12}^{(1)}$, the momentum conservation implies that $v_{1}+v_{2}=u_{1}+u_{2}=$ $1-v_{3}$. To verify this condition, one can rescale the momentum $u$ and $v$ in (5.17) making use of (3.21).

In this way, we obtain the following expression for the gauge field contribution to the twoparticle kernel, $\mathbb{V}_{12}^{(1)}$, coming from the diagrams shown in Fig. 4

$$
\mathbb{V}_{\text {gluon }}^{(1)}\left(u_{1}, u_{2} \mid v_{1}, v_{2}\right)=\frac{\delta\left(u_{1}+u_{2}-v_{1}-v_{2}\right)}{u_{1}+u_{2}}\left(\mathbb{V}_{\mathrm{QCD}}^{(1)}+\mathbb{V}_{\mathrm{SV}}^{(1)}\right)\left(\frac{u_{1}}{u_{1}+u_{2}}, \frac{v_{1}}{v_{1}+v_{2}}\right)
$$




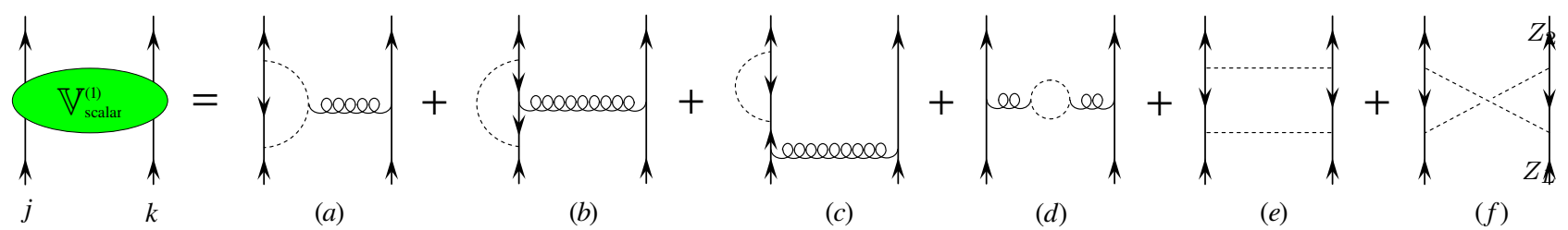

Figure 6: Two-particle irreducible contribution involving scalars in internal lines.

where $\mathbb{V}_{\mathrm{SV}}^{(1)}$ is defined in (5.24) and $\mathbb{V}_{\mathrm{QCD}}^{(1)}$ is given by the QCD expression (5.19) with the color factors substituted as

$$
\begin{array}{ll}
\text { - } \mathrm{QCD}: & C_{F} \rightarrow \frac{2}{3}, \quad N_{c} \rightarrow 3 \\
\text { - } \mathrm{SYM} \mathrm{:} & C_{F} \rightarrow N_{c} / 2, \quad n_{f} \rightarrow N_{c} \mathcal{N}
\end{array}
$$

Notice that the $\mathbb{V}_{A}^{(1)}$-term, Eqs. (5.19) and (5.21), only contributes in QCD and vanishes for gaugino in the adjoint representation. As we will show in Sect. 6. this leads to a dramatic difference in integrability properties of the two-loop dilatation operator in QCD and SYM theories.

The two-particle kernel $\mathbb{V}_{\text {gauge }}^{(1)}$ does not depend on the renormalization scheme employed - it is the same within the regularization by means of DREG or DRED provided that the perturbation series is in terms of $\overline{\mathrm{MS}}$-coupling constant. Since it more natural to define the coupling constant in regularization via DRED in the $\overline{\mathrm{DR}}$-scheme, the two-particle dilatation operator acquires an extra term as explained below in Sect. 5.2.3.

\subsubsection{Scalar contribution}

The scalars contribute to the two-particle evolution kernel in the $\mathcal{N}=2$ and $\mathcal{N}=4$ SYM theories. The corresponding Feynman diagrams are shown in Fig. 6. As before, the ladder diagram in Fig. 66 (e) vanishes due to the maximal $R$-charge of the three-fermion operator. The color factors of the diagrams in Fig. [6 (a), (b), (c) and (f) coincide with those shown in Fig. 2 (c), (d), (e) and (b), respectively, and can be read from the Table given above. In particular, the diagram in Fig. [6 (f) has a vanishing color factor and does not contribute. The remaining diagrams take the form of the one-loop graph (see Fig. (1) with the propagators and the vertices "dressed" by the scalar interaction. Therefore, it is not surprising that their contribution is proportional to the one-loop $f$-function (5.14) decorated by additional logarithms

$$
\begin{aligned}
\mathbb{V}_{\text {scalar }}^{(1)}\left(u_{1}, u_{2} \mid v_{1}, v_{2}\right)=\frac{n_{s}}{6} \delta\left(u_{1}+\right. & \left.u_{2}-v_{1}-v_{2}\right)\left\{\theta\left(v_{1}-u_{1}\right)\left(\ln \frac{u_{1}}{v_{1}}-\frac{4}{3}\right) f\left(u_{1}, v_{1}\right)\right. \\
+ & \left.\theta\left(v_{2}-u_{2}\right)\left(\ln \frac{u_{2}}{v_{2}}-\frac{4}{3}\right) f\left(u_{2}, v_{2}\right)\right\},
\end{aligned}
$$

with $n_{s}=2(\mathcal{N}-1)$ being the total number of scalars, Eq. (2.6). The kernel (5.27) is the same in the $\overline{\mathrm{MS}}-$ and $\overline{\mathrm{DR}}-$ schemes.

Notice that the scalar contribution (5.27) is similar to the $V_{\beta}$-term, Eq. (5.22). In the gluon contribution, Eqs. (5.19) and (5.25), this term is accompanied by the prefactor $\sim \beta_{\mathrm{QCD}, 0}=$ 
$11 N_{c} / 3-2 n_{f} / 3$, which coincides upon substitution (5.26) with the one-loop beta-function in SYM theory modulo the scalar contribution given by $-n_{s} / 6$. Combining together (5.25) and (5.27), one finds that the logarithmically enhanced part of the scalar contribution (5.27) is accompanied by the factor $+n_{s} / 6$. Writing this factor as $-n_{s} / 6+n_{s} / 3$ and splitting the scalar contribution into two terms, one finds that the first term forces the coefficient in front to the $V_{\beta}$-term to be equal to the complete one-loop beta-function in the SYM. As we will show in Sect. 6.1.4, the second term is an artefact of the $\overline{\mathrm{MS}}$-renormalization scheme used in the evaluation of (5.27).

\subsubsection{Two-particle kernel}

Let us combine together various two-loop contributions to the two-particle kernel $\mathbb{V}_{12}^{(1)}$, Eq. (5.2). They come from three different sources: the gluon kernel (5.25), scalar kernel (5.27) and twoparticle kernel induced by the subtraction terms in (5.16)

$$
\mathbb{V}_{12}^{(1)}=\mathbb{V}_{\text {gluon }}^{(1)}\left(u_{1}, u_{2} \mid v_{1}, v_{2}\right)+\mathbb{V}_{\text {scalar }}^{(1)}\left(u_{1}, u_{2} \mid v_{1}, v_{2}\right)+\mathbb{V}_{\text {sub }}^{(1)}\left(u_{1}, u_{2} \mid v_{1}, v_{2}\right)
$$

To identify the last term one substitutes the three-particle kernel (5.16) into (5.2) and selects the two-particle contribution proportional to $\delta\left(u_{3}-v_{3}\right)=\delta\left(u_{1}+u_{2}-v_{1}-v_{2}\right)$. In this way one gets

$$
\mathbb{V}_{\mathrm{sub}}^{(1)}\left(u_{1}, u_{2} \mid v_{1}, v_{2}\right)=\delta\left(u_{1}+u_{2}-v_{1}-v_{2}\right)\left[W\left(u_{1}, u_{2} \mid v_{1}, v_{2}\right)+W\left(u_{2}, u_{1} \mid v_{2}, v_{1}\right)\right]
$$

where

$$
W\left(u_{1}, u_{2} \mid v_{1}, v_{2}\right)=\int_{0}^{1} d u_{3}^{\prime} \mathbb{V}_{123}^{(1)}\left(u_{1}, 1-u_{1}-u_{3}^{\prime}, u_{3}^{\prime} \mid v_{1}, v_{2}, v_{3}\right)
$$

with $u_{2}=v_{1}+v_{2}-u_{1}$ and $v_{1}+v_{2}+v_{3}=1$. The explicit form of $\mathbb{V}_{\text {sub }}^{(1)}\left(u_{1}, u_{2} \mid v_{1}, v_{2}\right)$ can be found in Appendix B.

The two-particle kernel (5.28), contrary to its three-particle counterpart, is not universal and depends on the gauge theory under consideration. Substituting (5.25), (5.27) and (5.29) into (5.28) one finds after some algebra

$$
\begin{aligned}
\mathbb{V}_{12}^{(1)}=\delta\left(u_{1}+u_{2}-v_{1}-v_{2}\right) & {\left[\theta\left(\bar{u}_{2}\right) \theta\left(u_{2}-v_{1}-v_{2}\right) \mathcal{F}_{1}(\boldsymbol{u} \mid \boldsymbol{v})\right.} \\
+ & \left.\theta\left(u_{1}\right) \theta\left(v_{1}-u_{1}\right) \mathcal{F}_{2}(\boldsymbol{u} \mid \boldsymbol{v})+\frac{5}{8} \theta\left(u_{2}\right) \theta\left(v_{1}-u_{2}\right) \mathcal{F}_{3}(\boldsymbol{u} \mid \boldsymbol{v})+\left\{\begin{array}{l}
u_{1} \rightleftarrows u_{2} \\
v_{1} \rightleftarrows v_{2}
\end{array}\right\}\right],
\end{aligned}
$$

where $\bar{u}_{j} \equiv 1-u_{j}$ and we used the results $(\mathrm{B} .8)-(\mathrm{B} .9)$ from Appendix $\mathrm{B}$. The $\{\ldots\}-$ term ensures symmetry of the two-particle kernel under interchange of two particles. The $\mathcal{F}$-functions which enter as coefficients in front of different step-functions arise either from the subtraction terms alone $\left(\mathcal{F}_{1}\right)$ or the genuine two-particle irreducible kernel $\left(\mathcal{F}_{3}\right)$ or the sum of both $\left(\mathcal{F}_{2}\right)$. The former being defined by the three-particle irreducible kernel is given by a universal expression

$$
\begin{aligned}
\mathcal{F}_{1}(\boldsymbol{u} \mid \boldsymbol{v})=\frac{\bar{u}_{2}}{\left(\bar{v}_{1}-v_{2}\right)\left(v_{1}-u_{1}\right)} & -\frac{1}{2} L\left(-u_{1}, \bar{v}_{1}-v_{2}, v_{2}-u_{2}\right)-\frac{1}{2} L\left(-u_{1}, \bar{v}_{1}-v_{2}, v_{1}\right) \\
& +\frac{1}{2} L\left(u_{2}-v_{2}, \bar{v}_{2}, \bar{v}_{1}-v_{2}\right)+\frac{1}{2} L\left(u_{2}-v_{2}, \bar{v}_{2}, v_{1}\right)
\end{aligned}
$$

with $L(u, v, w)=\frac{1}{2 w} \ln \frac{u}{v}\left[4+\ln \frac{u}{v}\right]$. The two remaining functions are defined as follows: 
- In SYM theory with $\mathcal{N}$ supercharges one has

$$
\begin{aligned}
& \left.\mathcal{F}_{2}(\boldsymbol{u} \mid \boldsymbol{v})\right|_{\mathrm{SYM}}=\mathcal{F}_{0}(\boldsymbol{u} \mid \boldsymbol{v})+\frac{1}{2}\left(\frac{\beta_{\mathcal{N}, 0}}{N_{c}}+\mathcal{N}-1\right)\left[\ln \frac{u_{1}}{v_{1}}+\frac{5}{3}\right] f\left(u_{1}, v_{1}\right)-(\mathcal{N}-1) f\left(u_{1}, v_{1}\right), \\
& \left.\mathcal{F}_{3}(\boldsymbol{u} \mid \boldsymbol{v})\right|_{\mathrm{SYM}}=0
\end{aligned}
$$

where $\beta_{\mathcal{N}, 0}=N_{c}(4-\mathcal{N})$ is the one-loop beta-functions in SYM, Eqs. (2.9), and

$$
\begin{aligned}
\mathcal{F}_{0}(\boldsymbol{u} \mid \boldsymbol{v})= & \frac{1}{2} L\left(u_{2}-v_{2}, \bar{v}_{2}, \bar{v}_{1}-v_{2}\right)+\frac{1}{2} L\left(u_{2}-v_{2}, \bar{v}_{2}, v_{1}\right)-\frac{1}{2} L\left(u_{2}-v_{2}, v_{1}, \bar{v}_{1}-v_{2}\right) \\
& +\frac{1}{v_{1}}-\frac{1}{4 v_{1}} \ln ^{2}\left(1-\frac{u_{1}}{v_{1}}\right)+\left[\frac{2}{3}-\frac{\pi^{2}}{6}-\frac{3}{2} \ln \frac{u_{1}}{v_{1}}+\frac{1}{4} \ln ^{2} \frac{u_{1}}{v_{1}}\right] f\left(u_{1}, v_{1}\right) \\
& -\frac{1}{2}\left[f\left(u_{1}, v_{1}\right)-f\left(u_{2}, v_{2}\right)\right] \ln \frac{u_{1}}{v_{1}} \ln \left(1-\frac{u_{1}}{v_{1}}\right)+\frac{v_{1}+v_{2}}{2 v_{1} v_{2}} \ln \frac{u_{1}}{v_{1}+v_{2}} \ln \frac{u_{2}}{v_{1}+v_{2}}
\end{aligned}
$$

with the $f$-function defined in (5.14).

- In QCD the same functions are given by

$$
\begin{aligned}
\left.\mathcal{F}_{2}(\boldsymbol{u} \mid \boldsymbol{v})\right|_{\mathrm{QCD}} & =\mathcal{F}_{0}(\boldsymbol{u} \mid \boldsymbol{v})+\frac{3}{8} \beta_{\mathrm{QCD}, 0}\left[\ln \frac{u_{1}}{v_{1}}+\frac{5}{3}\right] f\left(u_{1}, v_{1}\right)+\frac{5}{8} \mathcal{F}_{\mathrm{QCD}}(\boldsymbol{u} \mid \boldsymbol{v}) \\
\left.\mathcal{F}_{3}(\boldsymbol{u} \mid \boldsymbol{v})\right|_{\mathrm{QCD}} & =-\frac{u_{1}}{v_{2}\left(v_{1}+v_{2}\right)}+\left[f\left(u_{2}, v_{2}\right)-f\left(u_{1}, v_{1}\right)\right]\left[2 \operatorname{Li}_{2}\left(1-\frac{u_{2}}{v_{2}}\right)+\ln ^{2} \frac{v_{2}}{v_{1}+v_{2}}\right] \\
& +2 f\left(u_{2}, v_{2}\right)\left[\operatorname{Li}_{2}\left(\frac{v_{1}}{v_{1}+v_{2}}\right)-\ln \frac{u_{2}}{v_{1}+v_{2}} \ln \frac{v_{2}}{v_{1}+v_{2}}\right]+2 f\left(u_{1}, v_{1}\right) \operatorname{Li}_{2}\left(\frac{u_{1}}{v_{1}+v_{2}}\right)
\end{aligned}
$$

where $\beta_{\mathrm{QCD}, 0}=11-\frac{2}{3} n_{f}$ is one-loop beta-functions in QCD, Eq. (2.8), and

$$
\begin{aligned}
\mathcal{F}_{\mathrm{QCD}}=\frac{4}{3} f\left(u_{1}, v_{1}\right)-\frac{u_{1}}{v_{1}\left(v_{1}+v_{2}\right)} & +2 f\left(u_{2}, v_{2}\right) \ln \frac{u_{2}}{v_{1}+v_{2}} \ln \frac{v_{1}}{v_{1}+v_{2}} \\
& -2 f\left(u_{1}, v_{1}\right)\left[\operatorname{Li}_{2}\left(\frac{u_{1}}{v_{1}+v_{2}}\right)+\operatorname{Li}_{2}\left(\frac{v_{2}}{v_{1}+v_{2}}\right)\right] .
\end{aligned}
$$

The following comments are in order.

The step-function structure in front of $\mathcal{F}_{1}$ looks quite unusual and even appears not to contribute to the moments (3.22) which are defined with the measure $[d u]_{3}$ possessing the support region $\left\{0 \leq u_{i} \leq 1, \sum_{i} u_{i}=1\right\}$. If one uses the momentum-conserving condition from the $\delta$ function accompanying $\mathcal{F}_{1}$ to express $\theta\left(u_{2}-v_{1}-v_{2}\right)$ as $\theta\left(-u_{1}\right)$ one would immediately come to this conclusion. This is not the case however since this contribution emerged from the subtraction term in the definition of the double plus-distribution in Eq. (5.16). As it is clear from there, while the $\mathbb{V}_{123}$-kernel itself resides on the simplices (4.16) and (4.18), the subtraction terms "live" in the entire domain of $u_{i}$. Their contribution to the two-particle subchannels was reconstructed from the subtracted expression by "integrating-in" corresponding $\delta$-functions and assuming that the support of the resulting subtracted kernel coincides with the original unsubtracted one. Therefore, the inconsistency which arises for the $\mathcal{F}_{1}$-term only is simply resolved by ignoring the spectral constraint on the total sum of momentum fractions after integrating out the momentum-conserving $\delta$-function in the measure $[d u]_{3}$. Namely,

$$
\int_{0}^{1}[d u]_{3} \delta\left(u_{1}+u_{2}-v_{1}-v_{2}\right) \theta\left(\bar{u}_{2}\right) \theta\left(u_{2}-v_{1}-v_{2}\right) \mathcal{F}_{1}(\boldsymbol{u} \mid \boldsymbol{v})=\int_{v_{1}+v_{2}}^{1} d u_{2} \mathcal{F}_{1}\left(v_{1}+v_{2}-u_{2}, u_{2}, v_{3} \mid v_{1}, v_{2}, v_{3}\right),
$$


with $v_{3}=1-v_{1}-v_{2}$ on the right-hand side. Analogously, one treats other terms with the $\mathcal{F}_{1}$ structure.

The expression (5.31) was obtained within the $\overline{\mathrm{MS}}$-renormalization scheme. Going over to the $\overline{\mathrm{DR}}$-scheme, one finds that contribution of all diagrams except the one containing gluon self-energy, Fig. 4 (f), remain the same. For the latter diagram, the difference between the two schemes arises at the level of $\mathcal{O}(\varepsilon)$ corrections to the gluon self-energy (see Fig. 51). These corrections interfere with a double pole coming from the Feynman integral to produce a nontrivial contribution to the two-particle evolution kernel which is proportional to the one-loop evolution kernel. We will return to this issue in Sect. 5.4.1.

Comparing (5.33) and (5.35) one notices that the two-particle kernel in SYM theories has a simpler form and, most importantly, it does not involve the $\mathrm{Li}_{2}$-function. This property is nontrivial since $\mathrm{Li}_{2}$ enters into the expression for gluon kernel (5.25) through the abelian contribution $\mathbb{V}_{\mathrm{SV}}^{(1)}$, Eq. (5.24). A close examination reveals that in the expression for the two-particle kernel (5.28) the $\mathrm{Li}_{2}$-terms coming from gluon kernel cancel against similar terms generated by subtraction term (5.29). Similar cancellation takes place inside the QCD expression (5.35) but in that case $\mathrm{Li}_{2}$-term also comes from the nonplanar kernel $\mathbb{V}_{A}^{(1)}$. In SYM theories this term does not contribute to the three-particle operators since it is accompanied by the color factor $C_{F}-N_{c} / 2$ which vanishes upon the substitution (5.23).

As was mentioned in Sect. 2, the conformal symmetry is broken in gauge theories with nonvanishing beta-function. One observes that the kernels (5.33) and (5.35) contain terms proportional to the one-loop beta-function. In the light-like axial gauge, they come from the one-loop Feynman diagram in which the internal gluon gets "dressed" by a one-loop self-energy correction (see Fig. 4 (f)). The corresponding terms are responsible for explicit breaking of conformal invariance of the evolution kernels at two loops. Other more subtle symmetry breaking effects will be addressed below in Sect. 6.1.4.

In distinction with the three-particle irreducible kernel (5.7), the two-particle kernels (5.33) and (5.35) are singular for $u_{j} \rightarrow v_{j}$. Carefully examining (5.31) in this limit, one finds that the two-particle kernel $\mathbb{V}_{12}^{(1)}$ develops simple poles $\sim 1 /\left(u_{j}-v_{j}\right)$ and, as a consequence, its moments are divergent. In the axial gauge with the principal value prescription, these poles are regularized according to (4.3) and lead to $\sim \ln \delta$ contribution to the moments. These singular terms can be separated with the help of the plus-distribution as in Eq. (4.15). For the pair-wise kernels $\mathbb{V}_{12}^{(1)}=\mathbb{V}^{(1)}\left(u_{1}, u_{2} \mid v_{1}, v_{2}\right)$ it reads

$$
\left[\mathbb{V}^{(1)}\left(u_{1}, u_{2} \mid v_{1}, v_{2}\right)\right]_{+}=\mathbb{V}^{(1)}\left(u_{1}, u_{2} \mid v_{1}, v_{2}\right)-\delta\left(u_{1}-v_{1}\right) \delta\left(u_{2}-v_{2}\right) f d u_{1} d u_{2} \mathbb{V}^{(1)}\left(u_{1}, u_{2} \mid v_{1}, v_{2}\right)
$$

where the integral in the right-hand side is evaluated using the principal value prescription. The two-particle kernel $\left[\mathbb{V}_{12}^{(1)}\right]_{+}$defined in this way has finite moments and satisfies the polynomiality condition.

In the SYM theories with $\mathcal{N}$ supercharges, the $\mathcal{N}$-dependence enters into the two-particle kernel (5.31) through the last two terms in the right-hand side of (5.33). Taking into account that $\beta_{\mathcal{N}, 0}=(4-\mathcal{N}) N_{c}$ one finds that the $\mathcal{N}$-dependence only resides in the last term proportional to the one-loop $f$-function, Eq. (5.14). Together with (4.17) this implies that the two-particle kernels in SYM theories with different number of supercharges are related to each other in a simple manner

$$
\left.\mathbb{V}_{12}^{(1)}\right|_{\mathcal{N}}=\left.\mathbb{V}_{12}^{(1)}\right|_{\mathcal{N}=1}-(\mathcal{N}-1) \mathbb{V}_{12}^{(0)}
$$




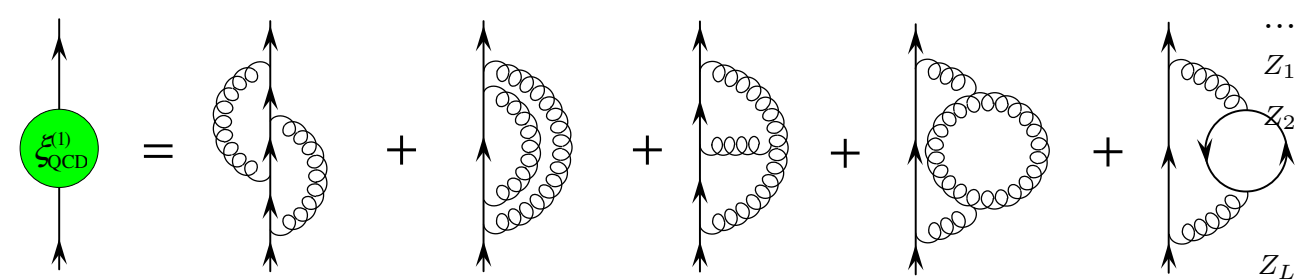

Figure 7: Two-loop fermion wave functions renormalization in QCD and $\mathcal{N}=1 \mathrm{SYM}$.

where $\mathbb{V}_{12}^{(0)}=\mathbb{V}^{(0)}\left(u_{1}, u_{2} \mid v_{1}, v_{2}\right)$ is the one-loop expression, Eq. (4.17). This suggests that the two-loop dilatation operators for different $\mathcal{N}$ should be related to each other in a simple manner (see Eq. (5.64) below).

\subsection{One-particle contributions}

Finally let us consider single-particle irreducible contributions $\mathbb{V}_{i}^{(1)}$ to the dilatation operator (5.2). They come from the fermion wave function renormalization and are defined by the Feynman diagrams shown in Figs. 7 and 8 .

The two-loop renormalization constants $\xi_{i}^{(1)}$ in Eq. (5.4) depend on the renormalization scheme used for their evaluation. In addition, in the light-like axial gauge $A_{+}(x)=0$ supplemented with the principal value prescription (4.3), they depend on the ratio of the light-cone momentum carried by the fermion and the regularization parameter $\delta$

$$
\xi_{i}^{(1)}=\xi^{(1)}\left(v_{i} / \delta\right) .
$$

The result of the calculation in the $\overline{\mathrm{MS}}$-scheme of $\xi^{(1)}(v / \delta)$ both in QCD [39] and SYM theories is

$$
\begin{aligned}
\xi_{\mathrm{QCD}}^{(1)}\left(k_{+} / \delta\right) & =\frac{N_{c}}{C_{F}}\left(\frac{17}{24}+\frac{11}{3} \zeta(2)-3 \zeta(3)+\left(\frac{67}{9}-2 \zeta(2)\right) \ln \frac{\delta}{k_{+}}\right) \\
& +\frac{3}{8}-3 \zeta(2)+6 \zeta(3)-\frac{n_{f}}{C_{F}}\left(\frac{1}{12}+\frac{2}{3} \zeta(2)+\frac{10}{9} \ln \frac{\delta}{k_{+}}\right), \\
\xi_{\mathcal{N}}^{(1)}\left(k_{+} / \delta\right) & =1+3 \zeta(3)+\left(\frac{19}{3}-2 \zeta(2)\right) \ln \frac{\delta}{k_{+}}+\frac{n_{s}^{2}}{8}-n_{s}\left(\frac{11}{12}+\ln \frac{\delta}{k_{+}}\right) .
\end{aligned}
$$

We recall that for three-quark operators in QCD the color factors take the values $C_{F}=4 / 3$ and $N_{c}=3$.

In Eq. (5.2), the self-energy correction (5.40) is accompanied by the delta-functions $\delta\left(u_{1}-\right.$ $\left.v_{1}\right) \delta\left(u_{2}-v_{2}\right)$. Similar contribution also comes from the subtraction terms entering into the definition the double-plus distribution for three-particle kernel (5.16) and from the plus distribution for two-particle kernel (5.38). Combining them together one gets

$$
\Gamma^{(1)}=\frac{1}{3} \int[d u]_{3} \mathbb{V}_{123}^{(1)}(\boldsymbol{u} \mid \boldsymbol{v})-\frac{1}{3} f d u_{1} d u_{2} \mathbb{V}_{12}^{(1)}\left(u_{1}, u_{2} \mid v_{1}, v_{2}\right)-\frac{1}{6}\left[\xi^{(1)}\left(v_{1} / \delta\right)+\xi^{(1)}\left(v_{2} / \delta\right)\right]+\ldots
$$

where ellipsis denote the terms needed to restore the symmetry under cyclic permutations of particles $\left(v_{1}, v_{2}, v_{3}\right) \rightarrow\left(v_{2}, v_{3}, v_{1}\right) \rightarrow\left(v_{3}, v_{1}, v_{2}\right)$. Two comments follow. First, notice that the 


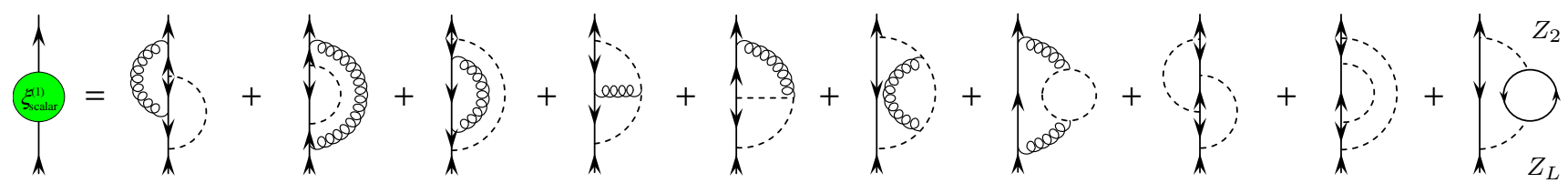

Figure 8: Two-loop Feynman graphs for wave function renormalization involving scalar fields propagating in internal lines.

integrals of three- and two-particle irreducible kernels in the right-hand side of (5.43) contain a nontrivial dependence on momentum fractions $v_{i}$. However, in the combination (5.43) this dependence entirely cancels as a manifestation of the restoration of the polynomiality condition. Second, the contributions of the two-particle kernel and self-energy corrections contain an explicit dependence on the axial gauge regularization parameter $\delta$. The gauge invariance requires that the divergences $\sim \ln \delta$ should cancel in their sum. Indeed, one can verify that $\Gamma^{(1)}$ stays finite for $\delta \rightarrow 0$. It reads in the $\overline{\mathrm{MS}}$ scheme for the QCD case with $N_{c}=3$

$$
\Gamma_{\mathrm{QCD}}^{(1)}=\frac{27}{16}+\frac{13}{32} \beta_{\mathrm{QCD}, 0},
$$

and for the SYM case

$$
\Gamma_{\mathrm{SYM}}^{(1)}=\frac{4}{3}-\frac{1}{12} n_{s}-\frac{1}{8} n_{s}^{2},
$$

with $n_{s}=2(\mathcal{N}-1)$. By the construction, $3 \Gamma^{(1)}$ defines the two-loop correction to the anomalous dimension of the local three-fermion operators, Eqs. (3.13) and (3.15), in the $\overline{\mathrm{MS}}-$ scheme. The one-loop correction is given by (4.12).

\subsection{Two-loop result}

Summarizing the calculation, the two-loop correction to the dilatation operator (5.2) reads

$\mathbb{V}^{(1)}=\left[\mathbb{V}_{123}^{(1)}\right]_{++}+\left[\mathbb{V}_{231}^{(1)}\right]_{++}+\left[\mathbb{V}_{312}^{(1)}\right]_{++}+\left[\mathbb{V}_{12}^{(1)}\right]_{+}+\left[\mathbb{V}_{23}^{(1)}\right]_{+}+\left[\mathbb{V}_{31}^{(1)}\right]_{+}-3 \Gamma^{(1)} \delta\left(u_{1}-v_{1}\right) \delta\left(u_{2}-v_{2}\right)$,

where each term in the right-hand side has finite moments and satisfies the polynomiality condition. Here the three-particle kernel $\mathbb{V}_{123}^{(1)}$ is defined in (5.7), the two-particle kernel $\mathbb{V}_{12}^{(1)}$ is given by (5.31) whereas the remaining kernels can be obtained from these two with the help of (5.3). The '++'- and '+'-distributions were introduced in (5.16) and (5.38), respectively. The single-particle contribution is specified in (5.44) and (5.45).

\subsubsection{Scheme dependence}

The above mentioned results were obtained within the $\overline{\mathrm{MS}}$-renormalization scheme. As was already mentioned, the two-particle and single-particle contributions have different form in the $\overline{\mathrm{MS}}-$ and $\overline{\mathrm{DR}}$-schemes. In QCD both schemes are equally suitable while in the SYM theory the $\overline{\mathrm{DR}}$-scheme is advantageous as it preserves supersymmetry. Therefore, as a final step we have to transform (5.46) to the supersymmetry preserving dimensional reduction scheme. This scheme 
transformation is achieved via a finite renormalization of the nonlocal light-cone operators (2.15) renormalized according to the conventional $\overline{\mathrm{MS}}$ scheme. For nonlocal operator of arbitrary length $L$ it reads

$$
\mathbb{O}_{\overline{\mathrm{DR}}}\left(z_{1}, \ldots, z_{L}\right)=w\left(g^{2}\right) \mathbb{O}_{\overline{\mathrm{MS}}}\left(z_{1}, \ldots, z_{L}\right),
$$

where $w\left(g^{2}\right)$ is, in general, a complicated integral operator acting on $z$-coordinates. Substituting this relation into the evolution equation (2.13) one finds that the evolution kernels in the two schemes are related as

$$
\mathbb{H}_{\overline{\mathrm{DR}}}\left(g^{2}\right)=w\left(g^{2}\right) \mathbb{H}_{\overline{\mathrm{MS}}}\left(g^{2}\right) w^{-1}\left(g^{2}\right)-\beta\left(g^{2}\right) \frac{\partial w\left(g^{2}\right)}{\partial g^{2}} w^{-1}\left(g^{2}\right) .
$$

Notice that in the conformal limit, for $\beta\left(g^{2}\right)=0$, the two evolution kernels are related to each other through similarity transformation and, therefore, have the same eigenvalues. The latter determine the anomalous dimension of Wilson operators in the underlying gauge theory.

Equation (5.46) defines the two-loop correction to $\mathbb{H}_{\overline{\mathrm{MS}}}\left(g^{2}\right)$ in the momentum representation. In order to transform it into the $\overline{\mathrm{DR}}$-scheme with the help of (5.48), it suffices to determine $w\left(g^{2}\right)$ to one loop. To this accuracy, $w$ arises due to the fact that the one-loop diagrams shown in Fig. 1 have different finite parts when renormalized in the $\overline{\mathrm{DR}}-$ and $\overline{\mathrm{MS}}-$ schemes. It is easy to see that for maximal-helicity operators, $w$ only receives contribution from the gluon self-energy, Fig. 团 (b), while the diagrams (a) and (c) are the same in both schemes to the $\sim \varepsilon^{0}$ accuracy. Therefore, $w$ is given by the unit operator times a factor. The latter can be easily computed with the result

$$
w\left(g^{2}\right)=1-L \frac{N_{c}}{4} \frac{g^{2}}{8 \pi^{2}} .
$$

Yet in the above scheme transformation, the gauge coupling is still defined in the $\overline{\mathrm{MS}}$ scheme. Thus, one has to further renormalize the coupling according to the DRED conventions, this yields its finite renormalization to two-loop accuracy [40]

$$
g_{\overline{\mathrm{MS}}}^{2}=g_{\overline{\mathrm{DR}}}^{2}\left(1-\frac{N_{c}}{6} \frac{g_{\mathrm{DR}}^{2}}{8 \pi^{2}}\right) .
$$

Thus, for the evolution kernel in the $\overline{\mathrm{MS}}$-scheme given by

$$
\mathbb{H}_{\overline{\mathrm{MS}}}=\lambda_{\overline{\mathrm{MS}}} \mathbb{H}^{(0)}+\lambda_{\overline{\mathrm{MS}}}^{2} \mathbb{H}^{(1)}+\mathcal{O}\left(\lambda^{3}\right),
$$

the same kernel in the $\overline{\mathrm{DR}}$-scheme takes the form

$$
\mathbb{H}_{\overline{\mathrm{DR}}}=\left(1-\frac{\lambda_{\overline{\mathrm{DR}}}}{6}\right)\left[\lambda_{\overline{\mathrm{DR}}} \mathbb{H}^{(0)}+\lambda_{\overline{\mathrm{DR}}}^{2} \mathbb{H}^{(1)}\right]-\frac{L}{4} \frac{\beta_{0}}{N_{c}} \lambda_{\overline{\mathrm{DR}}}^{2}+\mathcal{O}\left(\lambda^{3}\right),
$$

or equivalently

$$
\mathbb{H}_{\overline{\mathrm{DR}}}=\lambda_{\overline{\mathrm{DR}}} \mathbb{H}^{(0)}+\lambda_{\overline{\mathrm{DR}}}^{2}\left(\mathbb{H}^{(1)}-\frac{1}{6} \mathbb{H}^{(0)}-\frac{L}{4} \frac{\beta_{0}}{N_{c}}\right)+\mathcal{O}\left(\lambda^{3}\right) .
$$

According to (5.52), the two-loop dilatation operators in the two schemes differ by an overall normalization only and, therefore, they share the same symmetry properties (if any). This property is a unique feature of the dilatation operator in the maximal helicity sector and it holds in SYM theory independently of the number of supercharges $\mathcal{N}$. 
At $L=3$, one finds from (5.53) that the two-loop evolution kernel for three-particle operators in SYM theory is given in the $\overline{\mathrm{DR}}$-scheme in the momentum representation by

$$
\mathbb{V}_{\overline{\mathrm{DR}}}^{(1)}=\mathbb{V}^{(1)}-\frac{1}{6} \mathbb{V}^{(0)}-\frac{3}{4}(4-\mathcal{N}) \mathbb{1}
$$

with $\mathbb{V}^{(0)}$ and $\mathbb{V}^{(1)}$ defined in Eqs. (4.13) and (5.46) , respectively, while $\mathbb{1}$ is the identity operator $\mathbb{1}=\delta\left(u_{1}-v_{1}\right) \delta\left(u_{2}-v_{2}\right)$.

\subsubsection{Limiting cases}

The two-loop evolution kernel $\mathbb{V}^{(1)}$ is given by a rather complicated expression. There are however two limiting cases in which its form simplifies significantly. Let us examine $\mathbb{V}^{(1)}$ for $u_{j} \rightarrow v_{j}$ (with $j=1,2,3$ ). As was explained in Sect. 4.2, this corresponds to the limit when gluons exchanged between the fermions in the Feynman diagrams shown in Fig. 4 carry vanishing lightcone momenta $u_{j}-v_{j} \rightarrow 0$. We argued in Sect. 5.2.3, that for $u_{j} \rightarrow v_{j}$ the dominant contribution to $\mathbb{V}^{(1)}$ comes from the two-particle evolution kernels $\left[\mathbb{V}_{j k}^{(1)}\right]_{+}$which develop poles $\sim 1 /\left(u_{j}-v_{j}\right)$. It is straightforward to verify using (5.31) that these poles are generated by terms in the expression for $\mathbb{V}_{j k}^{(1)}$ involving the one-loop $f$-functions, Eq. (5.14),

$$
\mathbb{V}_{12}^{(1)} \stackrel{u_{j}}{\sim} v_{j} \delta\left(u_{1}+u_{2}-v_{1}-v_{2}\right)\left[\theta\left(v_{1}-u_{1}\right) f\left(u_{1}, v_{1}\right)+\theta\left(v_{2}-u_{2}\right) f\left(u_{2}, v_{2}\right)\right]
$$

One recognizes the expression in the right-hand side as the one-loop kernel $\mathbb{V}_{12}^{(0)}$ defined in (4.17). Substituting (5.55) into (5.46) one finds that for $u_{j}-v_{j} \rightarrow 0$ the full two-loop evolution kernel is proportional to the one-loop contribution, Eq. (4.13),

$$
\mathbb{V}(\boldsymbol{u} \mid \boldsymbol{v})=\lambda \mathbb{V}^{(0)}(\boldsymbol{u} \mid \boldsymbol{v})+\lambda^{2} \mathbb{V}^{(1)}(\boldsymbol{u} \mid \boldsymbol{v})+\mathcal{O}\left(\lambda^{3}\right) \stackrel{u_{j} \rightarrow v_{j}}{=} \frac{1}{2} \Gamma_{\text {cusp }}(\lambda) \cdot \mathbb{V}^{(0)}(\boldsymbol{u} \mid \boldsymbol{v})+\ldots,
$$

where ellipsis denote terms subleading as $u_{j}-v_{j} \rightarrow 0$. One can show that (5.56) holds to all orders of perturbation theory [41] with $\Gamma_{\text {cusp }}(\lambda)$ being the universal cusp anomalous dimension 42 ] related to a cusp anomaly of Wilson loops 43. The two-loop expression for $\Gamma_{\text {cusp }}(\lambda)$ can be obtained from (5.31). In SYM theory, in the $\overline{\mathrm{DR}}$-scheme one finds 44

$$
\Gamma_{\text {cusp }, \overline{\mathrm{DR}}}(\lambda)=2 \lambda+\lambda^{2}\left[2(4-\mathcal{N})-\frac{\pi^{2}}{3}\right]
$$

with the coupling constant $\lambda=g_{\overline{\mathrm{DR}}}^{2} N_{c} /\left(8 \pi^{2}\right)$.

The second limiting case corresponds to the selection of terms inside $\mathbb{V}(\boldsymbol{u} \mid \boldsymbol{v})$ proportional to the one-loop beta functions. These terms are gauge invariant and originate in the axial gauge from a special subset of Feynman diagrams involving gluon propagator dressed by one-loop corrections (see Fig. 4 (f)). In QCD one can automatically select the required terms by going over to the limit of large $\beta_{0}$, that is $g^{2} \beta_{0}=$ fixed for $\beta_{0} \rightarrow \infty$. Since $\beta_{0}=11-2 n_{f} / 3$, the large $\beta_{0}$-limit can be formally achieved in QCD by continuing $\mathbb{V}(\boldsymbol{u} \mid \boldsymbol{v})$ to the number of quark flavors $n_{f} \rightarrow-\infty$. Similar procedure can not be performed in the SYM theory since the number of gaugino equals the number of supercharges, Eq. (2.6). According to (5.31), the $\beta_{0}-$ term only comes from two-particle kernels and has the same form in QCD and SYM theories

$$
\mathbb{V}_{12}^{(1)}=\frac{\beta_{0}}{2 C_{R}} \delta\left(u_{1}+u_{2}-v_{1}-v_{2}\right)\left[\theta\left(u_{1}\right) \theta\left(v_{1}-u_{1}\right)\left(\ln \frac{u_{1}}{v_{1}}+\frac{5}{3}\right) f\left(u_{1}, v_{1}\right)+\left\{\begin{array}{l}
u_{1} \rightleftarrows u_{2} \\
v_{1} \rightleftarrows v_{2}
\end{array}\right\}\right]+\ldots
$$


where $C_{R}=N_{c}$ in SYM theories and $C_{R}=4 / 3$ in QCD. A unique feature of $\beta_{0}$ enhanced terms is that they can be resummed to all loops. To $n^{\text {th }}$ order of perturbation theory, these terms take the form $\lambda\left(\lambda \beta_{0}\right)^{n}$ and come from the one-loop Feynman diagrams in which the bare gluon propagator is dressed by "renormalon" chain involving $n$ one-loop polarization operators (see Fig. 51). These diagrams contribute to the two-particle kernels and lead to the following all-loop expression for the evolution kernel in the large $\beta_{0}$-limit

$$
\mathbb{V}(\boldsymbol{u} \mid \boldsymbol{v}) \stackrel{\beta_{0} \rightarrow \infty}{=} \mathbb{V}_{12}+\mathbb{V}_{23}+\mathbb{V}_{31}
$$

with

$$
\begin{aligned}
\mathbb{V}_{12} & =\lambda \mathbb{V}_{12}^{(0)}+\lambda^{2} \mathbb{V}_{12}^{(1)}+\lambda^{3} \mathbb{V}_{12}^{(2)}+\ldots=\lambda \varphi\left(\frac{\beta_{0} g^{2}}{16 \pi^{2}}\right) \delta\left(u_{1}+u_{2}-v_{1}-v_{2}\right) \\
& \times\left[\theta\left(u_{1}\right) \theta\left(v_{1}-u_{1}\right)\left(\frac{u_{1}}{v_{1}}\right)^{\frac{\beta_{0} g^{2}}{16 \pi^{2}}} f\left(u_{1}, v_{1}\right)+\left\{\begin{array}{l}
u_{1} \rightleftarrows u_{2} \\
v_{1} \rightleftarrows v_{2}
\end{array}\right\}\right],
\end{aligned}
$$

where $\lambda=g^{2} C_{R} /\left(8 \pi^{2}\right)$ and the notation was introduced for the function

$$
\varphi(x)=\frac{(1+x) \Gamma(4+2 x)}{6 \Gamma(1-x) \Gamma^{3}(2+x)} .
$$

It is easy to verify that the first two terms of the expansion of (5.60) in powers of $\lambda$ are in agreement with (5.58). Comparing (5.60) with the one-loop expression for the evolution kernel (4.17) one notices that higher order corrections "renormalize" the conformal spin $j$ of the fermion fields and induce the additional normalization factor [33, 45]

$$
\left.\mathbb{V}(\boldsymbol{u} \mid \boldsymbol{v}) \stackrel{\beta_{0} \rightarrow \infty}{=} \lambda \varphi\left(\frac{\beta_{0} g^{2}}{16 \pi^{2}}\right) \mathbb{V}^{(0)}(\boldsymbol{u} \mid \boldsymbol{v})\right|_{j=1+\frac{g^{2} \beta_{0}}{32 \pi^{2}}} .
$$

This relation holds both in QCD and SYM theories.

\subsection{3 $\mathcal{N}$-dependence}

To one-loop order, the kernel $\mathbb{V}^{(0)}$ depends on $\mathcal{N}$ through the normalization constant $\Gamma_{\text {SYM }}^{(0)}$, Eq. (4.12). To two-loop order, in Eq. (5.46), the $\mathcal{N}$-dependence resides in the two-particle kernels $\mathbb{V}_{j k}^{(1)}$ and the normalization constant $\xi^{(1)}$. Taking into account (5.39), one finds

$$
\mathbb{V}_{\mathcal{N}}^{(1)}=\mathbb{V}_{\mathcal{N}=1}^{(1)}-(\mathcal{N}-1)\left[\mathbb{V}_{\mathcal{N}=1}^{(0)}+\text { const }\right]
$$

where the subscript refers to the number of supercharges. This leads to the following remarkable relation between the two-loop dilatation operators in SYM theories with different number of supercharges

$$
\mathbb{H}_{\mathcal{N}}(\lambda)=[1-(\mathcal{N}-1) \lambda] \cdot \mathbb{H}_{\mathcal{N}=1}(\lambda)+\text { const }+\mathcal{O}\left(\lambda^{3}\right)
$$

We conclude that to two-loop accuracy the spectrum of anomalous dimensions in the sector of maximal helicity operators looks alike in all SYM theories including the maximally supersymmetric $\mathcal{N}=4$ theory. If the dilatation operators in these theories have different properties, the difference could only appear starting from three loops. Eq. (5.64) is one of the main results of this paper. 


\section{Eigenspectrum of the dilatation operator}

In the previous section, we have calculated the two-loop dilatation operator in the momentum representation. Let us now turn to the analysis of its symmetry properties. Our strategy will be to solve the spectral problem for the two-loop evolution kernel $\mathbb{V}(\boldsymbol{u} \mid \boldsymbol{v})$, Eq. (3.25), and, then, look for the hidden symmetry of its spectrum.

As we already observed in Sect. 3.1. the dilatation operator is invariant under translations and dilatations along the light-ray, Eqs. (3.12) and (3.11), respectively. The latter are the light-cone projections of the Poincaré transformations in the underlying gauge theory. In the momentum representation, this symmetry leads to scaling property of the evolution kernel (3.21) which in its turn allows one to identify its eigenstates as homogeneous polynomials, Eq. (3.28). It is well-known that classical Yang-Mills theory is invariant under an even larger set of space-time transformations - the $S O(4,2)$ conformal group. The conformal symmetry imposes additional constraints both on the properties of the evolution kernels and their eigenspectra. In particular, it allows one to resolve the mixing between the Wilson operators involving total derivatives 46. 32 .

On quantum level, the conformal symmetry becomes anomalous for $\beta\left(g^{2}\right) \neq 0$. As we will explain later in this section, the conformal anomaly affects the dilatation operator starting from two loops only and, therefore, the one-loop dilatation operator, Eqs. (4.7) and (4.13), inherits the conformal symmetry of the classical theory. We will demonstrate that the conformal symmetry allows one to diagonalize the two-particle evolution kernels in the coordinate and momentum representations, Eqs. (4.11) and (4.17), respectively, but it is not sufficient to diagonalize the three-particle dilatation operators.

Before we turn to the three-particle operators, let us examine the spectrum of the dilatation operators for the twist-two operators, Eqs. (2.18) and (2.17). The reason for doing this is twofold. Firstly, the evolution kernel for twist-two operators contributes to the two-particle kernel $\mathbb{V}_{12}$ in the expression for the evolution kernel (5.46). Secondly, this offers a convenient framework for discussing the conformal symmetry breaking.

\subsection{Twist-two operators}

The generating function for twist-two operators in QCD and SYM theories is defined in (2.18) and (2.17), respectively. Similar to (3.27), the twist-two operators are uniquely defined by polynomials $P\left(u_{1}, u_{2}\right)$ which are eigenstates of the evolution kernel in the momentum representation, $\mathbb{V}\left(u_{1}, u_{2} \mid v_{1}, v_{2}\right)$. In QCD the two-loop evolution kernel $\mathbb{V}_{\mathrm{QCD}}\left(u_{1}, u_{2} \mid v_{1}, v_{2}\right)$ was defined in (5.17). In SYM theories the evolution kernel $\mathbb{V}_{\mathrm{SYM}}\left(u_{1}, u_{2} \mid v_{1}, v_{2}\right)$ can be obtained from the QCD expression by properly adjusting the color factors and adding the contribution of scalars determined in Sect. 5.2 .2 .

\subsubsection{Eigenspectrum at one-loop}

To one-loop order, the evolution kernel for twist-two maximal-helicity operators in QCD and SYM are given by the same universal expression (5.18)

$$
\mathbb{V}_{\mathrm{tw}-2}^{(0)}(\boldsymbol{u} \mid \boldsymbol{v})=2\left[\mathbb{V}^{(0)}\left(u_{1}, u_{2} \mid v_{1}, v_{2}\right)\right]_{+}-2 \Gamma^{(0)} \delta\left(u_{1}-v_{1}\right) \delta\left(u_{2}-v_{2}\right),
$$


with $\Gamma^{(0)}$ defined in (4.12) and $\mathbb{V}^{(0)}$ being the two-particle evolution kernel, Eq. (4.14). Solving the spectral problem for this operator

$$
\int[d u]_{2}\left[\mathbb{V}^{(0)}\left(u_{1}, u_{2} \mid v_{1}, v_{2}\right)\right]_{+} P_{n}^{(\ell)}\left(u_{1}, u_{2}\right)=-\gamma(n) P_{n}^{(\ell)}\left(v_{1}, v_{2}\right),
$$

with $[d u]_{2}=d u_{1} d u_{2} \delta\left(u_{1}+u_{2}-v_{1}-v_{2}\right)$, it is straightforward to verify that its eigenstates are given by Gegenbauer polynomials [26, 47]

$$
P_{n}^{(\ell)}\left(u_{1}, u_{2}\right)=\left(u_{1}+u_{2}\right)^{n+\ell} \mathrm{C}_{n}^{3 / 2}\left(\frac{u_{1}-u_{2}}{u_{1}+u_{2}}\right)
$$

with $n$ and $\ell$ nonnegative integers. The corresponding eigenvalues do not depend on $\ell$ and are given by the Euler digamma function

$$
\gamma(n)=2[\psi(n+2)-\psi(2)]
$$

The eigenstates of the two-particle evolution kernel, Eq. (6.3), define the local twist-two operators $\mathcal{O}_{n l}(0)$ with $l \geq n$

$$
\mathcal{O}_{n l}(0)=\left.P_{n}^{(l-n)}\left(\partial_{1}, \partial_{2}\right) \mathbb{O}\left(z_{1}, z_{2}\right)\right|_{z_{1}=z_{2}=0},
$$

where $\mathbb{O}\left(z_{1}, z_{2}\right)$ is given by (2.18) and (2.17). As follows from (6.3), the operators $\mathcal{O}_{n l}(0)$ with $l>n$ can be obtained from the one with $l=n$ by applying the total derivatives, $\mathcal{O}_{n l}(0)=$ $\partial_{+}^{l-n} \mathcal{O}_{n n}(0)$.

The operators (6.5) have an autonomous scale dependence to one-loop order

$$
\mu \frac{d}{d \mu} \mathcal{O}_{n l}(0)=-\frac{g^{2}}{8 \pi^{2}} \gamma_{n}^{(0)} \mathcal{O}_{n l}(0)+\mathcal{O}\left(g^{4}\right),
$$

with the anomalous dimension given in QCD and in the SYM theory with $\mathcal{N}$ supercharges by

$$
\begin{aligned}
& \left.\gamma_{n}^{(0)}\right|_{\mathrm{QCD}}=C_{F}[4 \psi(n+2)-4 \psi(2)+1], \\
& \left.\gamma_{n}^{(0)}\right|_{\mathrm{SYM}}=N_{c}[4 \psi(n+2)-4 \psi(2)+\mathcal{N}] .
\end{aligned}
$$

Although these expressions define the anomalous dimensions for integer $n \geq 0$, they can be analytically continued to the complex $n$-plane. In particular, for $n=-1$ one finds in the SYM case that the anomalous dimension coincides with the one-loop beta-function, Eq. (2.9),

$$
\gamma_{n=-1}^{(0)}=-\beta_{\mathcal{N}, 0}=N_{c}[\mathcal{N}-4]
$$

As we will argue in Sect.6.1.5, this leading order property is not accidental and can be extended to all loops.

\subsubsection{Conformal symmetry}

As was already mentioned, the explicit form of the one-loop eigenstates (6.3) is uniquely determined by the conformal symmetry. We remind that the nonlocal light-cone operators $\mathbb{O}\left(z_{1}, z_{2}\right)$ are built from elementary fields $X\left(z n_{\mu}\right)$ located on the light ray, defined by the null vector $n_{\mu}$. For such operators the full $S O(4,2)$ conformal group consisting of Lorentz rotations $\mathbb{M}_{\mu \nu}$, translations $\mathbb{P}_{\mu}$, dilatation $\mathbb{D}$ and special conformal boosts $\mathbb{K}_{\mu}$ is effectively reduced to its "collinear" 
$S L(2)$ subgroup which acts nontrivially on functions of the light-cone coordinate $z=x \cdot n^{*}$, defined by means of a tangent vector $n_{\mu}^{*}$ to the light cone such that $n \cdot n^{*}=1$. The generators of the collinear subgroup are

$$
\mathbb{L}^{-}=-i \mathbb{P}_{+}, \quad \mathbb{L}^{+}=\frac{i}{2} \mathbb{K}_{-}, \quad \mathbb{L}^{0}=\frac{i}{2}\left(\mathbb{M}_{-+}+\mathbb{D}\right),
$$

with the standard commutation relations

$$
\left[\mathbb{L}^{0}, \mathbb{L}^{ \pm}\right]= \pm \mathbb{L}^{ \pm}, \quad\left[\mathbb{L}^{+}, \mathbb{L}^{-}\right]=\mathbb{L}^{0}
$$

These quantum field operators can be expressed in terms of the energy-momentum tensor in the underlying gauge theory. Still, for their action on the elementary field operators $X\left(z_{k}\right) \equiv X\left(z_{k} n_{\mu}\right)$ they can be represented by differential operators acting on the light-cone coordinate

$$
\begin{aligned}
{\left[\mathbb{L}^{-}, X\left(z_{k}\right)\right] } & \equiv L_{k}^{-} X\left(z_{k}\right)=-\partial_{z_{k}} X\left(z_{k}\right), \\
{\left[\mathbb{L}^{+}, X\left(z_{k}\right)\right] } & \equiv L_{k}^{+} X\left(z_{k}\right)=\left(z_{k}^{2} \partial_{z_{k}}+2 z_{k} j\right) X\left(z_{k}\right), \\
{\left[\mathbb{L}^{0}, X\left(z_{k}\right)\right] } & \equiv L_{k}^{0} X\left(z_{k}\right)=\left(z_{k} \partial_{z_{k}}+j\right) X\left(z_{k}\right),
\end{aligned}
$$

where the notation was introduced for the differential operators $L_{k}^{\alpha}$ (with $\alpha= \pm$, 0 ) acting on the light-cone coordinate $z_{k}$. Here $j$ is the conformal spin of the field constructed from its scaling dimension $d$ and the projection of the spin onto the light-cone $s$

$$
j=\frac{1}{2}(d+s) .
$$

For gaugino/quark operators defined in (2.14) and (2.16), these parameters admit the values at the classical level $d=3 / 2$ and $s=1 / 2$ leading to $j=1$. In quantum theory, the scaling dimension $d$ acquires an anomalous contribution while $s$ is protected to all orders of the perturbation theory.

The field $X\left(z_{k}\right)$ defines a representation of the $S L(2 ; \mathbb{R})$ group which we shall denote as $\mathcal{V}_{j}$. The representation space $\mathcal{V}_{j}$ possesses the highest weight $X(0)$, such that $L^{+} X(0)=0$ and $L^{0} X(0)=j X(0)$ while the descendant states are given by its derivative $\left(L^{-}\right)^{k} X(0)=$ $(-1)^{k} \partial_{+}^{k} X(0)$. The nonlocal operators $\mathbb{O}\left(z_{1}, z_{2}\right)$ belong to the tensor product of two $S L(2 ; \mathbb{R})$ modules which can be decomposed over the irreducible components as

$$
\mathcal{V}_{j} \otimes \mathcal{V}_{j}=\sum_{n \geq 0} \mathcal{V}_{2 j+n}
$$

The representation space of $\mathcal{V}_{2 j+n}$ is spanned by local twist-two Wilson operators $\mathcal{O}_{n l}(0)$. Among these operators one can distinguish the highest weight $l=n$ satisfying the relations [48, 49]

$$
\left[\mathbb{L}^{+}, \mathcal{O}_{n n}(0)\right]=0, \quad\left[\mathbb{L}^{0}, \mathcal{O}_{n n}(0)\right]=(2 j+n) \mathcal{O}_{n 0}(0)
$$

and its descendants with $l>n$

$$
\left.\left.\mathcal{O}_{n l}(0)=\left[\left[\mathcal{O}_{n n}(0), \mathbb{L}^{-}\right], \mathbb{L}^{-}\right] \ldots \mathbb{L}^{-}\right]\right]=\left(\partial_{+}\right)^{l-n} \mathcal{O}_{n n}(0)
$$

The operators $\mathcal{O}_{n l}(0)$ carry the conformal spin $2 j+n$ and have the canonical dimension $l+3$.

It is straightforward to verify that the twist-two operators defined in (6.5) and (6.3) satisfy the relations (6.15) and (6.16) and, therefore, they belong to the $S L(2 ; \mathbb{R})$ module $\mathcal{V}_{2 j+n}$. Then, 
the conformal invariance implies that the Wilson operators belonging to different $S L(2)$ modules do not mix with each other and, in addition, the operators belonging to the same module have the same anomalous dimension.

Let us define the representation of the conformal group generators for the nonlocal light-cone operators $\mathbb{O}\left(z_{1}, z_{2}\right)$ similar to (6.12). Evaluating $\left[\mathbb{L}^{\alpha}, \mathbb{O}\left(z_{1}, z_{2}\right)\right]$ (with $\alpha= \pm, 0$ ) and making use of (6.12), it is easy to see that to the lowest order of perturbation theory the generators $\mathbb{L}^{\alpha}$ are given by the sum of differential operators $L_{1}^{\alpha}+L_{2}^{\alpha}$ defined in (6.12). In two of these operators, $\mathbb{L}^{-}$ and $\mathbb{L}^{0}$, we immediately recognize (up to an additive constant) the differential operators which were found to commute with the dilatation operator, see Eqs. (3.11) and (3.12). The conformal symmetry implies that the one-loop dilatation operator also commutes with the conformal boost $\mathbb{L}^{+}$leading to

$$
\left[L_{1}^{\alpha}+L_{2}^{\alpha}, \mathbb{H}_{12}^{(0)}\right]=0,
$$

with $\alpha= \pm$, 0 . Notice that in distinction with (3.11) and (3.12) this property is lost starting from two loops due to the conformal anomaly. We remind that the evolution kernel $\mathbb{V}^{(0)}\left(u_{1}, u_{2} \mid v_{1}, v_{2}\right)$ defines the operator $\mathbb{H}_{12}^{(0)}$, Eq. (4.11), in the momentum representation.

It follows from (6.17) that the two-particle evolution kernel $\mathbb{H}_{12}^{(0)}$ is a function of the quadratic Casimir operators defined on the tensor product (6.14)

$$
\begin{aligned}
L_{12}^{2} & =\left(L_{12}^{0}\right)^{2}+\frac{1}{2}\left(L_{12}^{+} L_{12}^{-}+L_{12}^{-} L_{12}^{+}\right) \\
& =-\left(z_{1}-z_{2}\right)^{2(1-j)} \partial_{z_{1}} \partial_{z_{2}}\left(z_{1}-z_{2}\right)^{2 j}=J_{12}\left(J_{12}-1\right)
\end{aligned}
$$

with $L_{12}^{\alpha}=\left(L_{1}+L_{2}\right)^{\alpha}$ and $J_{12}$ being the two-particle conformal spin. In the coordinate representation, the $S L(2)$ module $V_{2 j+n}$ is spanned by homogeneous polynomials

$$
\Psi_{n}^{(0)}\left(z_{1}, z_{2}\right)=\left(z_{1}-z_{2}\right)^{n}, \quad \Psi_{n}^{(\ell)}\left(z_{1}, z_{2}\right)=\left(L_{12}^{+}\right)^{\ell} \Psi_{n}^{(0)}\left(z_{1}, z_{2}\right) .
$$

with $\Psi_{n}^{(0)}$ the lowest weight, $L_{12}^{-} \Psi_{n}^{(0)}=0$, and $\Psi_{n}^{(\ell)}$ its descendant. These polynomials are in the one-to-one correspondence with similar polynomials in the momentum representation, Eq. (6.3), and satisfy the orthogonality condition similar to (3.26). The eigenstates (6.19) diagonalize the Casimir operator (6.18) and, as a consequence, the Hamiltonian (4.11) has the same eigenvalues on these eigenfunctions independent of $\ell$,

$$
\begin{aligned}
\mathbb{H}_{12}^{(0)} \cdot \Psi_{n}^{(\ell)}\left(z_{1}, z_{2}\right) & =2[\psi(n+2 j)-\psi(2 j)] \Psi_{n}^{(\ell)}\left(z_{1}, z_{2}\right), \\
L_{12}^{2} \Psi_{n}^{(\ell)}\left(z_{1}, z_{2}\right) & =(n+2 j)(n+2 j-1) \Psi_{n}^{(\ell)}\left(z_{1}, z_{2}\right)
\end{aligned}
$$

with $\psi(x)=d \ln \Gamma(x) / d x$. This relation is a counter-part of (6.2) in the momentum representation. Together with $J_{12} \cdot \Psi_{n}^{(\ell)}=(n+2 j) \Psi_{n}^{(\ell)}$ it allows one to write down the two-particle kernel in the $S L(2)$ invariant form

$$
\mathbb{H}_{12}^{(0)}=2\left[\psi\left(J_{12}\right)-\psi(2 j)\right] .
$$

Here $j=1$ is the conformal spin of the quark/gaugino fields to the lowest order of perturbation theory.

\subsubsection{Conformal symmetry breaking}

Let us now examine the two-loop corrections to the dilatation operator for the twist-two operators. As was explained in Sect. 5.4.1, starting from two loops the dilatation operator depends 
on the renormalization scheme. Throughout the paper we employ the $\overline{\mathrm{MS}}-$ scheme and its spinoff the $\overline{\mathrm{DR}}$-scheme. Another subtle issue emerging at two loops is the breaking of conformal symmetry in the dilatation operator within these two schemes even for $\beta\left(g^{2}\right)=0$.

It is a matter of traditional folklore that in a generic gauge theory the conformal symmetry is broken if the beta-function is nonvanishing. This is a direct consequence of the dimensional transmutation phenomenon which generates an intrinsic mass scale in gauge theory modifying the scaling behavior of correlation functions. The nonzero beta-function induces an anomaly in the trace of the energy-momentum tensor. The latter is then generates in turn anomalous dimensions of composite operators when their product is renormalized in the conformal Ward identities, as we will demonstrate below. However, even in cases when the four-dimensional beta function is zero to all orders of perturbation theory, there is yet another source of the conformal symmetry breaking related to the choice of the regularization procedure. We remind that in the dimensional regularization with $D=4-2 \varepsilon$ the beta-function acquires an additional contribution $\beta_{\varepsilon}\left(g^{2}\right) \sim-2 \varepsilon$, Eq. (4.6), and, therefore, the conformal symmetry is violated for $\varepsilon \neq 0$. According to (4.4) and (4.5), the dilatation operator is related to the residue of a simple pole $1 / \varepsilon$ in the expression for renormalized light-cone operator $\mathbb{O}\left(z_{i}\right)$. Subtracting divergences and sending $\varepsilon \rightarrow 0$ afterwards, one generates symmetry breaking contribution to the dilatation operator coming from terms $\sim \beta_{\varepsilon}\left(g^{2}\right) / \varepsilon$. This source of the symmetry breaking is a peculiar feature of dimensional regularization rather than intrinsic property of the dilatation operator. In other words, in gauge theories with vanishing beta-function the conformal symmetry breaking terms can be removed by performing a scheme transformation of the dilatation operator and by going over to the so-called conformal scheme. Obviously, this transformation does not affect the eigenvalues of the dilatation operator but it does change the form of the corresponding eigenstates.

The conformal operators $\mathbb{O}_{n l}(0)$ define the basis of twist-two operators in gauge theory. To one-loop order these operators have an autonomous scale dependence but they mix starting from two loops

$$
\left(\mu \frac{\partial}{\partial \mu}+\beta\left(g^{2}\right) \frac{\partial}{\partial g^{2}}\right) \mathcal{O}_{n l}(0)=-\sum_{k=0}^{n} \gamma_{n k}\left(g^{2}\right) \mathcal{O}_{k l}(0) .
$$

The mixing matrix is given by the perturbative expansion

$$
\gamma_{n k}\left(g^{2}\right)=\frac{g^{2}}{8 \pi^{2}} \gamma_{n}^{(0)} \delta_{n k}+\left(\frac{g^{2}}{8 \pi^{2}}\right)^{2} \gamma_{n k}^{(1)}+\mathcal{O}\left(g^{6}\right)
$$

and its form to higher orders is constrained by the Lorentz symmetry. To see this, one considers the operator $\mathcal{O}_{n n}\left(z n_{\mu}\right)$ having the canonical dimension $n+3$ and carrying the maximal possible conformal spin. It could mix with operators $\mathcal{O}_{m n}\left(z n_{\mu}\right)$ of the same canonical dimension but smaller conformal spin $m<n$. Notice that these operators necessarily involve $(n-m)$ total derivatives. Then, expanding the operators around the origin $z=0$, one finds that $\mathcal{O}_{n l}(0)=$ $\partial_{+}^{l-n} \mathcal{O}_{n n}(0)$ could only mix with the operators $\mathcal{O}_{k l}(0)$ with $k \leq n$. This means that the matrix of anomalous dimensions $\gamma_{n k}\left(g^{2}\right)$ has a triangular form, $\gamma_{n k}=0$ for $n<k$, or explicitly

$$
\gamma^{(1)}=\left(\begin{array}{ccc}
\gamma_{00}^{(1)} & & 0 \\
\vdots & \ddots & \\
\gamma_{n 0}^{(1)} & \cdots & \gamma_{n n}^{(1)}
\end{array}\right) \equiv \gamma_{n}^{(1)} \delta_{n k}+\gamma_{n k}^{\mathrm{ND}} \theta_{n k},
$$


with the diagonal entries $\gamma_{n}^{(1)} \equiv \gamma_{n n}^{(1)}$ and non-diagonal elements accompanied by the step function $\theta_{n k}=\{1, n>k ; 0, n \leq k\}$. The eigenvalues of the mixing matrix (6.24) are only determined by the diagonal matrix elements $\gamma_{n}^{(1)}$ while the off-diagonal matrix elements only affect the form of the corresponding eigenstates.

A convenient approach to study manifestation of the conformal symmetry breaking in the mixing matrix (6.24) is through the conformal Ward identities. Below we briefly review this formalism, for a detailed discussion see [50] and reviews [46, 32. Let us examine the variation of the correlation function $\left\langle\mathcal{O}_{n l}(0) X\left(z_{1}\right) \ldots X\left(z_{L}\right)\right\rangle$ under transformations generated by the $S L(2)$ generator $\mathbb{L}^{0}$

$$
\begin{aligned}
\sum_{k}^{L}\left(j+z_{k} \partial_{k}\right)\left\langle\mathcal{O}_{n l}(0) X\left(z_{1}\right) \ldots X\left(z_{L}\right)\right\rangle & \equiv-\left\langle\mathcal{O}_{n l}(0) \delta_{0}\left(X\left(z_{1}\right) \ldots X\left(z_{L}\right)\right)\right\rangle \\
= & \left\langle\delta_{0} \mathcal{O}_{n l}(0) X\left(z_{1}\right) \ldots X\left(z_{L}\right)\right\rangle+\left\langle i \delta_{0} S_{\mathrm{YM}} \mathcal{O}_{n l}(0) X\left(z_{1}\right) \ldots X\left(z_{L}\right)\right\rangle
\end{aligned}
$$

with $\delta_{0} X\left(z_{k}\right)=-\left[\mathbb{L}^{0}, X\left(z_{k}\right)\right]$ defined in (6.12). The last term in the right-hand side is due to nonvanishing variation of the regularized Yang-Mills action. It is generated by a nonvanishing trace of the energy-momentum tensor in the $D$-dimensional gauge theory $\delta_{0} S_{\mathrm{YM}}=-i \int d^{D} z \Theta_{\mu \mu}(z)$. The product of the conformal operator and the trace anomaly requires an additional renormalization. This produces an anomalous contribution proportional to the anomalous dimension of the conformal operator $\mathcal{O}_{n l}$

$$
\mathcal{O}_{n l}(0) \delta_{0} S_{\mathrm{YM}}=\frac{i}{2} \gamma_{n k}\left(g^{2}\right) \mathcal{O}_{k l}(0)+\ldots
$$

where ellipsis denote "regular" terms. Here and in what follows the summation over the repeated index $0 \leq k \leq n$ is implied. Combining together (6.25) and (6.26) one obtains that the conformal operator is transformed as

$$
\left[\mathcal{O}_{n l}(0), \mathbb{L}^{0}\right]=-\frac{1}{2}\left[(l+4 j) \delta_{n k}+\gamma_{n k}\left(g^{2}\right)\right] \mathcal{O}_{k l}(0)-\frac{i}{2}\left[\mathcal{O}_{n l}(0) \int d^{4} z \Theta_{\mu \mu}(z)\right]_{\mathrm{R}}+\ldots
$$

where $j$ is the conformal spin of the constituent field and the subscript ' $\mathrm{R}$ ' indicates that the product of two operators is renormalized. In a similar manner, one can examine the variation of correlation functions under transformations generated by the $S L(2)$ generator $\mathbb{L}^{+}$. They lead to the following commutation relations

$$
\left[\mathcal{O}_{n l}(0), \mathbb{L}^{+}\right]=\frac{i}{2}\left[a_{n k}(l)+\gamma_{n k}^{c}\left(l ; g^{2}\right)\right] \mathcal{O}_{k l-1}-\frac{i}{2}\left[\mathcal{O}_{n l}(0) \int d^{4} z 2 z^{-} \Theta_{\mu \mu}(z)\right]_{\mathrm{R}}+\ldots
$$

where $a_{n k}(l) \equiv a(n, l) \delta_{n k}=2(n-l)(n+l+4 j-1) \delta_{n k}$ is a diagonal matrix and the matrix $\gamma_{n k}^{c}\left(l ; g^{2}\right)$ is the so-called special conformal anomaly. This matrix has a well-defined perturbative expansion in powers of the gauge coupling and similar to the scale anomaly, Eq. (6.26), it arises from the renormalization of the product of the conformal operator and the variation of the Yang-Mills action,

$$
\mathcal{O}_{n l}(0) \delta_{+} S_{\mathrm{YM}}=\frac{1}{2} \gamma_{n k}^{c}\left(l ; g^{2}\right) \mathcal{O}_{k l-1}+\ldots
$$

The one-loop expression for $\gamma_{n k}^{c}$ has been found in Ref. [50]. 
We remind that the $S L(2)$ generators satisfy the commutation relation $\left[\mathbb{L}^{0}, \mathbb{L}^{+}\right]=\mathbb{L}^{+}$. Evaluating the commutators with $\mathcal{O}_{n l}(0)$ on both sides of this relation and making use of (6.27) and (6.28) one obtains the consistency condition

$$
\left[\boldsymbol{\gamma}\left(g^{2}\right), \boldsymbol{a}+\boldsymbol{\gamma}^{c}\left(g^{2}\right)+\beta\left(g^{2}\right) \boldsymbol{b}\right]=0,
$$

which relates to each other the two anomaly matrices. Here, the $\boldsymbol{a}$-matrix was defined in (6.28) while the matrix $\boldsymbol{b}$ possesses the following off-diagonal elements $b_{n k}=-2(2 k+3) \theta_{n k}$. In distinction with $\gamma_{n k}\left(g^{2}\right)$, the matrix elements $\gamma_{n k}^{c}\left(l ; g^{2}\right)$ depend on the scaling dimension of the conformal operator $l$. This dependence can be fixed from the commutation relations $\left[\mathbb{L}^{0}, \mathbb{L}^{-}\right]=$ $\mathbb{L}^{-}$leading to

$$
\gamma_{n k}^{c}\left(l+1 ; g^{2}\right)-\gamma_{n k}^{c}\left(l ; g^{2}\right)=-2 \gamma_{n k}\left(g^{2}\right) .
$$

Since the one-loop correction to the matrix of anomalous dimension is diagonal, Eq. (6.23), the relation (6.30) is satisfied automatically to the lowest order of perturbation theory. Beyond leading order one finds that the special conformal anomaly has off-diagonal elements, $\gamma_{n k}^{c}\left(l ; g^{2}\right) \neq$ 0 for $n>k$, and as a consequence the anomalous dimension matrix $\gamma_{n k}\left(g^{2}\right)$ also acquires nonzero off-diagonal elements, even in theory with vanishing four-dimensional beta function. The reason for this apparently counterintuitive result is due to the fact that the beta-function is different from zero in $D=4-2 \varepsilon$ dimensions, the anomaly propagates into the finite result for the off-diagonal matrix elements of $\gamma_{n k}\left(g^{2}\right)$.

Expanding the left-hand side of (6.30) in powers of $g^{2}$ one observes that (6.30) mixes orders of perturbative expansion. Obviously, this relation does not provide any information on the diagonal elements of the anomalous dimension matrix $\gamma_{n n}$ but it does constrain the off-diagonal elements $\gamma_{n k}$ with $n>k$. In two-loop approximation, taking into account the explicit form of the one-loop special conformal anomaly matrix $\gamma_{n k}^{c}\left(l ; g^{2}\right)$, one finds the off-diagonal elements of the anomalous dimension matrix as 50

$$
\left.\gamma_{n k}^{(1)}\right|_{n>k}=-\left(\gamma_{n}^{(0)}-\gamma_{k}^{(0)}\right)\left\{d_{n k}\left(\gamma_{k}^{(0)}+\beta_{0}\right)+g_{n k}\right\},
$$

where the coefficients $d_{n k}$ are defined in terms of the derivative of the Gegenbauer polynomials over its index as

$$
\left.\frac{d}{d \nu} C_{n}^{\nu}(x)\right|_{\nu=3 / 2}=-2 \sum_{k=0}^{n} d_{n k} C_{k}^{3 / 2}(x), \quad d_{n k}=-\frac{2 k+3}{(n-k)(n+k+3)},
$$

while the explicit form of $g_{n k}$-coefficients is not relevant for our purposes and can be found in [50. As we show in the next section, Eq. (6.32) allows one to restore the contribution of scalars to the two-loop evolution kernels in SYM without actual loop calculations.

As we just explained, the conformal symmetry is broken in dimensional regularization even when the four-dimensional $\beta$-function vanishes identically. In the latter case, the conformal symmetry can be restored however by performing a finite renormalization of the conformal operators, Eq. (6.5)

$$
\mathcal{O}_{n l}(0)=\mathcal{B}_{n k}\left(g^{2}\right) \widehat{\mathcal{O}}_{k l}(0) \text {. }
$$

The $\mathcal{B}$-matrix absorbs symmetry breaking effects and rotates the conformal operators to a new basis, in which the operators $\widehat{\mathcal{O}}_{k l}(0)$ do not mix and have an autonomous scale dependence (for $\left.\beta\left(g^{2}\right)=0\right)$

$$
\mu \frac{d}{d \mu} \widehat{\mathcal{O}}_{n l}(0)=-\gamma_{n}\left(g^{2}\right) \widehat{\mathcal{O}}_{n l}(0)
$$


The anomalous dimensions $\gamma_{n}\left(g^{2}\right)$ coincide with the diagonal matrix elements of the mixing matrix (6.23), $\gamma_{n} \equiv \gamma_{n n}\left(g^{2}\right)$. The transformation (6.34) defines a new scheme known as the conformal subtraction scheme, or simply $\overline{\mathrm{CS}}$. By construction, the rotation matrix $\mathcal{B}$ diagonalizes the conformal anomaly matrix

$$
\left(\mathcal{B}^{-1} \gamma\left(g^{2}\right) \mathcal{B}\right)_{n k}=\gamma_{n}\left(g^{2}\right) \delta_{n k}
$$

Wherefrom one can find that $\mathcal{B}_{n k}^{-1}=\delta_{n k}+\gamma_{n k}^{c}\left(k ; g^{2}\right) / a(n, k)$. Then, it follows from (6.30) that, in the conformal limit $\beta\left(g^{2}\right)=0$, the $\mathcal{B}$-matrix also diagonalizes the special conformal anomaly matrix

$$
\left(\mathcal{B}^{-1}\left[\boldsymbol{a}+\boldsymbol{\gamma}^{c}\left(g^{2}\right)\right] \mathcal{B}\right)_{n k}=2(n-l)\left(n+l+4 j-1+\gamma_{n}\left(g^{2}\right)\right) \delta_{n k} .
$$

In this limit, the energy-momentum tensor has vanishing trace, $\Theta_{\mu \mu}=0$, and, as a consequence, Eqs. (6.27) and (6.28) simplify significantly. Combining them with (6.34), (6.36) and (6.37) we conclude that the operators $\widehat{\mathcal{O}}_{n l}(0)$ (with $l \geq n$ ) define a representation of the collinear $S L(2)$ group. The highest weight of this representation, $\widehat{\mathcal{O}}_{n n}(0)$, satisfies the relations

$$
\left[\mathbb{L}^{+}, \widehat{\mathcal{O}}_{n n}(0)\right]=0, \quad\left[\mathbb{L}^{0}, \widehat{\mathcal{O}}_{n n}(0)\right]=\left(n+2 j+\gamma_{n}\left(g^{2}\right)\right) \widehat{\mathcal{O}}_{n n}(0),
$$

which should be compared with (6.15). One observes that, in the conformal limit, higher order corrections renormalize the conformal spin of the $S L(2)$ module, to which the conformal operators $\widehat{\mathcal{O}}_{n l}(0)$ belong to, by an amount proportional to their anomalous dimension. This property alone fixes the explicit form of the operators $\widehat{\mathcal{O}}_{n l}(0)$. As before, the conformal symmetry constrains the form of twist-two operators but it does not allow us to determine the explicit expressions for their anomalous dimension.

\subsubsection{Contribution of scalars from one-loop special conformal anomaly}

Let us give a brief demonstration of the application of the conformal constraint (6.32) at twoloop order and how it compares with the conventional diagonalization procedure of the evolution kernels deduced in the previous sections. In QCD, the twist-two two-loop evolution kernel in the momentum representation was given in Eq. (5.17). In SYM case, the two-loop evolution kernel receives an additional contribution from scalars determined by the Feynman diagrams shown in Fig. 6. As was already explained in Sect. 5.2. the scalar contribution to the twist-two evolution kernel coincides, modulo the overall color factor, with the two-particle kernel (5.27) contributing to the three-fermion evolution kernel.

In the basis of conformal operators $\mathcal{O}_{n l}(0)$, Eqs. (6.5) and (6.3), the scalar kernel (5.27) is represented by the mixing matrix $\gamma_{n k}^{(1) \text { sc }}$. Similar to (6.2), it can be found as

$$
\int[d u]_{2} 2 \mathbb{V}_{\mathrm{scalar}}^{(1)}\left(u_{1}, u_{2} \mid v_{1}, v_{2}\right) \mathrm{C}_{n}^{3 / 2}\left(\frac{u_{1}-u_{2}}{u_{1}+u_{2}}\right)=-\sum_{k=0}^{n}\left(\gamma_{n k}^{(1) \mathrm{sc}} / N_{c}^{2}\right) \mathrm{C}_{k}^{3 / 2}\left(\frac{v_{1}-v_{2}}{v_{1}+v_{2}}\right)
$$

with the measure $[d u]_{2}$ defined after Eq. (6.2). Here, the factor 2 in the left-hand side accounts for the difference in color factors of singlet and octet kernels, cf. Table in Sect. [5.2.1. As expected, the mixing matrix has a triangular form, $\gamma_{n k}^{(1) s c}=0$ for $k>n$. Making use of (6.2), it is easy to see that the off-diagonal matrix elements $\gamma_{n k}^{(1) \mathrm{sc}}$ with $k<n$ are generated by the $\ln (u / v)$-enhanced terms in the right-hand side of (5.27). 
Let us demonstrate that the contribution of scalars to the two-loop mixing matrix $\gamma_{n k}^{(1) \text { sc }}$ is fully consistent with the conformal symmetry constraints (6.30) and (6.32). One notices that the scalars contribute to the right-hand side of (6.32) through the one-loop anomalous dimension, Eq. (6.8), and the one-loop beta-function

$$
\gamma_{n}^{(0)}=\frac{1}{2} n_{s} N_{c}+\ldots, \quad \beta_{0}=-\frac{1}{6} n_{s} N_{c}+\ldots
$$

where $n_{s}=2(\mathcal{N}-1)$ and ellipsis denote the remaining, $n_{s}$-independent terms. Substituting these expressions into (6.32), we can predict the contribution of scalars to the off-diagonal part of the two-loop mixing matrix

$$
\left.\gamma_{n k}^{(1) \mathrm{sc}}\right|_{n>k}=-\left(\gamma_{n}^{(0)}-\gamma_{k}^{(0)}\right) d_{n k}\left(-\frac{n_{s}}{6}+\frac{n_{s}}{2}\right) N_{c},
$$

where $\gamma_{n}^{(0)}$ is given by (6.8) and the coefficients $d_{n k}$ were defined in (6.33). A straightforward calculation of the integral in the left-hand side of (6.39) leads to the same expression.

\subsubsection{Anomalous dimension of twist-two operators}

Let us finally turn to the calculation of the eigenvalues of the two-loop mixing matrices in QCD and SYM theories. We remind that the mixing matrix has a triangular form (6.24) and, therefore, its eigenvalues coincide with the diagonal matrix elements, $\gamma_{n}\left(g^{2}\right)=\gamma_{n n}\left(g^{2}\right)$. The off-diagonal matrix elements correspond to the admixture of operators with total derivatives and they do not affect $\gamma_{n}\left(g^{2}\right)$. One can easily get rid of this redundant mixing by neglecting the operators with total derivatives. This can be rigorously done by going over from the operators to their forward matrix elements over some reference momentum state, the so-called forward limit

$$
\mathcal{O}_{n n}(0) \rightarrow\left\langle p\left|\mathcal{O}_{n n}(0)\right| p\right\rangle
$$

In distinction with $\mathcal{O}_{n n}(0)$ its forward matrix elements have an autonomous scale dependence since the operators with total derivatives do not contribute $\left\langle p\left|\mathcal{O}_{n n}(0)\right| p\right\rangle$. In the momentum representation, the forward limit amounts to setting the total momentum equal to zero, $\sum_{k} u_{k}=$ $\sum_{k} v_{k}=0$.

To two-loop order, the eigenvalue of the mixing matrix can be written as

$$
\gamma_{n}\left(g^{2}\right)=\frac{g^{2}}{8 \pi^{2}} \gamma_{n}^{(0)}+\left(\frac{g^{2}}{8 \pi^{2}}\right)^{2} \gamma_{n}^{(1)}+\ldots
$$

Here $\gamma_{n}^{(0)}$ is the same in the $\overline{\mathrm{MS}}-$ and $\overline{\mathrm{DR}}$-schemes while $\gamma_{n}^{(1)}$ is scheme-dependent. In QCD, the eigenvalue of the two-loop evolution kernel (5.19) can be extracted from the available expression for the anomalous dimension of the quark transversity operator in the $\overline{\mathrm{MS}}$ scheme 37 ]

$$
\begin{aligned}
\gamma_{n}^{(0)} & =C_{F}\left\{4 S_{1}(n)-3\right\} \\
\gamma_{n}^{(1), \overline{\mathrm{MS}}} & =C_{F}^{2}\left\{-\frac{3}{4}+6 S_{2}(n)+16 S_{-2,1}(n)-8 S_{3}(n)-8 S_{-3}(n)-8 S_{1}(n)\left(S_{2}(n)+2 S_{-2}(n)\right)\right\} \\
& +C_{F} N_{c}\left\{-\frac{17}{12}+\frac{134}{9} S_{1}(n)-\frac{22}{3} S_{2}(n)-8 S_{-2,1}(n)+4 S_{3}(n)+4 S_{-3}(n)+8 S_{1}(n) S_{-2}(n)\right\} \\
& +C_{F} n_{f}\left\{\frac{1}{6}-\frac{20}{9} S_{1}(n)+\frac{4}{3} S_{2}(n)\right\},
\end{aligned}
$$


for odd $n$. It is expressed in terms of the harmonic sums

$$
S_{ \pm \ell}(n)=\sum_{k=1}^{n+1} \frac{( \pm 1)^{k}}{k^{\ell}}, \quad S_{ \pm \ell, m}(n)=\sum_{k=1}^{n+1} \frac{( \pm 1)^{k}}{k^{\ell}} S_{m}(k-1) .
$$

The one-loop result (6.44) coincides with (6.7). The two-loop result can be reproduced by calculating the mixing matrix in the basis of conformal operators, $\gamma_{n k}$, and, then, finding its eigenvalues.

To obtain the eigenvalues of the two-loop mixing matrix in SYM case, we have to "supersymmetrize" the QCD expression (6.44) and (6.45) by adjusting the values of the color factors, then adding the contribution of scalars present in SYM theories with extended supersymmetry and, finally, transforming the entire result to the DRED scheme. The first of these steps is trivially accomplished via the identification

$$
C_{F} \rightarrow N_{c}, \quad n_{f} \rightarrow N_{c} \mathcal{N} .
$$

The anomalous dimension obtained via this procedure will be denoted $\gamma_{n}^{\mathrm{sCD}, \overline{\mathrm{MS}}}$. We remind that in SYM with $\mathcal{N}$ supercharges, the gaugino fields belong to the adjoint representation and their total number is related to the number of scalars as $n_{f}=1+n_{s} / 2=\mathcal{N}$. So we set it to this value in what follows.

Let us now add the contribution of scalars. To one- and two-loop orders it comes from diagrams shown in Fig. 1 (c) and Figs. 6, 8, respectively. These diagrams have been calculated in the previous section as a part of the evolution kernel for three-gaugino operators. For the twist-two operators, the only difference is that the pair of gaugino fields carries zero color charge which affects the overall color factors. Using our findings from the previous section, we get for the contribution of scalars in the $\overline{\mathrm{MS}}$ scheme

$$
\gamma_{n}^{\mathrm{sc}, \overline{\mathrm{MS}}}\left(g^{2}\right)=\frac{g^{2} N_{c}}{8 \pi^{2}} \frac{n_{s}}{2}+\left(\frac{g^{2} N_{c}}{8 \pi^{2}}\right)^{2}\left\{-\frac{n_{s}^{2}}{4}+n_{s}\left(\frac{7}{4}-\frac{8}{9} S_{1}(n)-\frac{2}{3} S_{2}(n)\right)\right\} .
$$

Combining this expression together with the QCD result "supersymmetrized" via (6.47), we get the anomalous dimension of the twist-two gaugino operator (2.17) in the $\overline{\mathrm{MS}}$-scheme

$$
\gamma_{n}^{\overline{\mathrm{MS}}}\left(g^{2}\right)=\gamma_{n}^{\mathrm{sQCD}, \overline{\mathrm{MS}}}\left(g^{2}\right)+\gamma_{n}^{\mathrm{sc}, \overline{\mathrm{MS}}}\left(g^{2}\right) .
$$

As a final step we have to transform this result to the supersymmetry preserving dimensional reduction scheme. This scheme transformation was described in detail in Sect. 5.3.1 for a general $L$-particle operator. Applying the formulas (5.53) for $L=2$ we obtain the anomalous dimension in the dimensional reduction scheme as

$$
\gamma_{n}^{\overline{\mathrm{DR}}}\left(g_{\overline{\mathrm{DR}}}^{2}\right)=\gamma_{n}^{\overline{\mathrm{MS}}}\left(g_{\overline{\mathrm{DR}}}^{2}-\frac{N_{c}}{6} \frac{g_{\mathrm{DR}}}{8 \pi^{2}}\right)-\frac{\beta_{0} N_{c}}{2}\left(\frac{g_{\overline{\mathrm{DR}}}^{2}}{8 \pi^{2}}\right)^{2}+\mathcal{O}\left(g^{6}\right) .
$$

Combining together (6.49) and (6.50), we find the one- and two-loop corrections to the anomalous dimension (6.43) in the SYM theory as functions of the number of supercharges $\mathcal{N}$

$$
\begin{aligned}
& \gamma_{n}^{(0)}= N_{c}\left\{\mathcal{N}-4+4 S_{1}(n)\right\}, \\
& \gamma_{n}^{(1), \overline{\mathrm{DR}}}=N_{c}^{2}\left\{-(\mathcal{N}-4)(\mathcal{N}-2)-4\left[\mathcal{N}-4+2 S_{2}(n)+2 S_{-2}(n)\right] S_{1}(n)\right. \\
&\left.\quad-4 S_{3}(n)-4 S_{-3}(n)+8 S_{-2,1}(n)\right\} .
\end{aligned}
$$


Here, the $\mathcal{N}$-dependence accounts for contribution from $n_{s}=2(\mathcal{N}-1)$ scalar and $n_{f}=\mathcal{N}$ gaugino fields. The one-loop result matches (6.8).

The following comments are in order.

In SYM theory, the maximal helicity twist-two operator (2.17) is a component of a supermultiplet of twist-two operators. Supersymmetry implies that all components of the supermultiplet have the same anomalous dimension, Eqs. (6.43) and (6.51). In the maximally supersymmetric $\mathcal{N}=4$ SYM theory, all twist-two operators belong to a single supermultiplet and, as a consequence, their anomalous dimension is given by the so-called universal anomalous dimension. ${ }^{8}$ One can verify that for $\mathcal{N}=4$ our result for the anomalous dimension, Eqs. (6.43) and (6.51), agrees with the one of Refs. [51, 52.

The expression for the two-loop anomalous dimension, Eqs. (6.43) and (6.51), can be analytically continued from nonnegative integer $n$ to the entire complex $n$-plane. The value of the anomalous dimension for $n=-1$ is of a special interest. Formally, it corresponds to an anomalous dimension of a nonlocal operator $\lambda^{\{A} \partial_{+}^{-1} \lambda^{B\}}$ with canonical dimension 2. However, supersymmetry implies that the same quantity determines the anomalous dimension of a local operator, given by the product of two complex holomorphic scalar fields. The latter operator is protected by supersymmetry and its anomalous dimension is given to all orders by the beta-function of the corresponding SYM model [53]. This leads to the following relation

$$
\gamma_{n=-1}^{\overline{\mathrm{DR}}}\left(g_{\overline{\mathrm{DR}}}^{2}\right)=\beta_{\mathrm{SYM}}\left(g_{\overline{\mathrm{DR}}}^{2}\right) .
$$

Indeed, one verifies using (6.46) that for $n=-1$ all harmonic sums vanish in the left-hand side of (6.51) and the relation (6.52) holds true to two loops.

We argued in Sect. 5.4.3, that the $\mathcal{N}$-dependence of the evolution kernel for three-gaugino operators can be factored out into c-valued normalization factors. One can verify that the same relation (5.64) fulfilled by the anomalous dimension of the twist-two operators, Eqs. (6.43) and (6.51). Moreover, taking into account (6.52) one can write this relation in a concise form as

$$
\left[\gamma_{n}\left(g^{2}\right)-\beta_{\mathrm{SYM}}\left(g^{2}\right)\right]_{\mathcal{N}}=\left(1-(\mathcal{N}-1) \frac{g^{2} N_{c}}{8 \pi^{2}}\right)\left[\gamma_{n}\left(g^{2}\right)-\beta_{\mathrm{SYM}}\left(g^{2}\right)\right]_{\mathcal{N}=1}+\mathcal{O}\left(g^{6}\right),
$$

where we introduced the subscript to indicate the value of $\mathcal{N}=0,1,2,4$. We conclude that the anomalous dimensions of twist-two operators (2.17) in all SYM theories are related to each other to two-loop accuracy as in (6.53). Taking into account that $\beta_{\mathrm{SYM}}\left(g^{2}\right)=0$ for $\mathcal{N}=4$, the same relation can be written as

$$
\left[\gamma_{n}\left(g^{2}\right)-\beta_{\mathrm{SYM}}\left(g^{2}\right)\right]_{\mathcal{N}}=\left(1-(\mathcal{N}-4) \frac{g^{2} N_{c}}{8 \pi^{2}}\right)\left[\gamma_{n}\left(g^{2}\right)\right]_{\mathcal{N}=4}+\mathcal{O}\left(g^{6}\right) .
$$

Let us examine the asymptotics of the anomalous dimension at large $n$. For $n \gg 1$ the leading contribution to (6.51) comes from terms involving $S_{1}(n) \sim \ln n$. In this way one finds that the anomalous dimension scales logarithmically at large $n$

$$
\gamma_{n}\left(g^{2}\right)=4\left(\frac{g^{2} N_{c}}{8 \pi^{2}}-\left(\frac{g^{2} N_{c}}{8 \pi^{2}}\right)^{2}\left[\mathcal{N}-4+\frac{\pi^{2}}{6}\right]\right) \ln n+\mathcal{O}\left(n^{0}\right) .
$$

\footnotetext{
${ }^{8}$ In SYM theory with $\mathcal{N}<4$ supercharges there are two different supermultiplets of twist-two operators and, as a consequence, there are two universal anomalous dimensions [24. The maximal helicity operators belong to one of the supermultiplets.
} 
Comparing this relation with (5.57) one observes that the prefactor coincides with the cusp anomalous dimension leading to

$$
\gamma_{n}\left(g^{2}\right)=2 \Gamma_{\text {cusp }}(\lambda) \ln n+\mathcal{O}\left(n^{0}\right),
$$

with the coupling $\lambda=g^{2} N_{c} /\left(8 \pi^{2}\right)$ defined in (4.10). One can show that this relation holds true to all loops with $\Gamma_{\text {cusp }}(\lambda)$ modified by higher order corrections [41. Substituting (6.56) into (6.53) one finds that as far as the $\mathcal{N}$-dependence is concerned the cusp anomaly satisfies the same relation, Eq. (6.53).

\subsection{Three-particle operators}

Let us now turn to diagonalization of the evolution kernel for the three-fermion operators in QCD and SYM theories. As for two particle operators, we start in Sect. 6.2.1 with finding the eigenspectrum of the dilatation operator to one-loop order. This allows us to elucidate in Sect. 6.2.2 the symmetries of the all-order dilatation operator and their manifestation in the eigenspectrum. Then in Sect. 6.2.3 we numerically study the eigenspectrum of the dilatation operator (5.46) at two loops and consider afterwards the limits of large spin and $\beta_{0} \rightarrow \infty$.

\subsubsection{Eigenspectrum to one-loop}

The one-loop dilatation operator has been determined in Sect. 4.2. We demonstrated there that it is given in QCD and SYM theories by the same universal expression. In the coordinate representation, the eigenproblem for the one-loop dilatation operator reads

$$
\mathbb{H}^{(0)} \Psi_{\boldsymbol{q}}\left(z_{1}, z_{2}, z_{3}\right)=\gamma_{\boldsymbol{q}}^{(0)} \Psi_{\boldsymbol{q}}\left(z_{1}, z_{2}, z_{3}\right)
$$

with the kernel $\mathbb{H}^{(0)}$ (4.7) built from the pairwise interactions (4.11) and $\boldsymbol{q}$ enumerating the solutions. In the momentum representation, the same relation looks like

$$
\int[d u]_{3} \mathbb{V}^{(0)}(\boldsymbol{u} \mid \boldsymbol{v}) P_{\boldsymbol{q}}\left(u_{1}, u_{2}, u_{3}\right)=-\gamma_{\boldsymbol{q}}^{(0)} P_{\boldsymbol{q}}\left(v_{1}, v_{2}, v_{3}\right)
$$

with the evolution kernel $\mathbb{V}^{(0)}(\boldsymbol{u} \mid \boldsymbol{v})$ given by (4.13) and the integration measure $[d u]_{3}$ defined in Eq. (3.19). The advantage of the coordinate representation is that the conformal symmetry is manifest (see Eq. (6.21)). On the other hand, the momentum representation is more convenient for actual evaluation of the eigenvalues $\gamma_{\boldsymbol{q}}^{(0)}$ since it allows one to eliminate the contribution of operators with total derivatives by going over to the forward limit $\sum_{k} u_{k}=\sum_{k} v_{k}=0$ (see Appendix A). The eigenstates in the two representations, $\Psi_{\boldsymbol{q}}\left(z_{i}\right)$ and $P_{\boldsymbol{q}}\left(v_{i}\right)$ are homogeneous polynomials in light-cone coordinates and momentum, respectively. They are related to each other through the orthogonality condition (3.26). We remind that $P_{\boldsymbol{q}}\left(v_{i}\right)$ determines the explicit form of local Wilson operator (3.27) while $\Psi_{\boldsymbol{q}}\left(z_{i}\right)$ defines the coefficient function accompanying this operator in the operator product expansion (3.4).

The dilatation operator $\mathbb{H}^{(0)}$, Eq. (4.7), is invariant under the cyclic permutation of particles, $\mathbb{P}$, and the interchange of any their pair, say $\mathbb{P}_{12}$,

$$
[\mathbb{P}, \mathbb{H}]=\left[\mathbb{P}_{12}, \mathbb{H}\right]=0
$$


with the corresponding discrete operators defined as

$$
\mathbb{P} \Psi_{\boldsymbol{q}}\left(z_{1}, z_{2}, z_{3}\right)=\Psi_{\boldsymbol{q}}\left(z_{2}, z_{3}, z_{1}\right), \quad \mathbb{P}_{12} \Psi_{\boldsymbol{q}}\left(z_{1}, z_{2}, z_{3}\right)=\Psi_{\boldsymbol{q}}\left(z_{2}, z_{1}, z_{3}\right)
$$

Since these two operators do not commute, $\left[\mathbb{P}, \mathbb{P}_{12}\right] \neq 0$, the eigenfunctions $\Psi_{\boldsymbol{q}}\left(z_{i}\right)$ can diagonalize either $\mathbb{P}$ or $\mathbb{P}_{12}$, but not both simultaneously. If $\Psi_{\boldsymbol{q}}\left(z_{i}\right)$ diagonalizes $\mathbb{P}$, then the eigenstates of (6.57) can be classified according to their quasimomentum $\theta_{\boldsymbol{q}}$,

$$
\mathbb{P} \Psi_{\boldsymbol{q}}\left(z_{1}, z_{2}, z_{3}\right)=\mathrm{e}^{i \theta_{\boldsymbol{q}}} \Psi_{\boldsymbol{q}}\left(z_{1}, z_{2}, z_{3}\right)
$$

which takes three different values satisfying $\mathrm{e}^{3 i \theta_{\boldsymbol{q}}}=1$

$$
\theta_{\boldsymbol{q}}=0, \pm \frac{2 \pi}{3}
$$

Then, the eigenfunctions of (6.57) with definite parity are given by

$$
\Psi_{\boldsymbol{q}}^{ \pm}(\boldsymbol{z})=\frac{1}{\sqrt{2}}\left(1 \pm \mathbb{P}_{12}\right) \Psi_{\boldsymbol{q}}(\boldsymbol{z}), \quad \mathbb{P}_{12} \Psi_{\boldsymbol{q}}^{ \pm}(\boldsymbol{z})= \pm \Psi_{\boldsymbol{q}}^{ \pm}(\boldsymbol{z})
$$

Invariance of the dilatation operator under the discrete transformations (6.59) immediately implies that its spectrum is double degenerate except for the eigenstates with zero quasimomentum $\theta_{\boldsymbol{q}}=0$ [3]. To see this one applies the eigenstate $\Psi_{\boldsymbol{q}}\left(z_{1}, z_{2}, z_{3}\right)$ to both sides of the relation $\mathbb{P} \mathbb{P}_{12} \mathbb{P}=\mathbb{P}_{12}$ which follows from the definition (6.60). In this way, one obtains

$$
\mathbb{P}\left(\mathbb{P}_{12} \Psi_{\boldsymbol{q}}\left(z_{i}\right)\right)=\mathrm{e}^{-i \theta \boldsymbol{q}} \mathbb{P}_{12} \Psi_{\boldsymbol{q}}\left(z_{i}\right)
$$

and, therefore, $\mathbb{P}_{12} \Psi_{\boldsymbol{q}}\left(z_{i}\right)$ defines yet another eigenstate with the same "energy" $\gamma_{\boldsymbol{q}}^{(0)}$ unless $\theta_{\boldsymbol{q}}=0$, leading to

$$
\left[\mathbb{H}^{(0)}-\gamma_{\boldsymbol{q}}(\lambda)\right] \Psi_{\boldsymbol{q}}^{ \pm}\left(z_{i}\right)=0
$$

As we will see in a moment, integrability extends this property to the eigenstates with $\theta_{\boldsymbol{q}}=0$. We would like to stress that the degeneracy of the eigenstates with $\theta_{\boldsymbol{q}} \neq 0$ follows from the symmetry of the dilatation operator under discrete transformations (6.60) and, therefore, it holds to all loops.

The additional restrictions on the eigenspectrum of the dilatation operator are imposed by the properties of the nonlocal light-cone operators (2.19) and (2.20). Since both operators are invariant under cyclic permutations of fields, the same property should hold true for the polynomials $\Psi_{\boldsymbol{q}}^{ \pm}\left(z_{i}\right)$ entering (3.4). Therefore, their quasimomentum ought to be equal to zero, $\theta_{\boldsymbol{q}}=0$. One can "revive" the remaining eigenstates with $\theta_{\boldsymbol{q}} \neq 0$ by assigning an additional "flavor" index to fermions. In QCD this procedure has a direct physical meaning (there are six different species of quarks) while in the SYM case it would violate supersymmetry by breaking a balance between bosonic and fermionic degrees of freedom.

The one-loop dilatation operator $\mathbb{H}^{(0)}$ is invariant under the conformal transformations, $\left[L_{1}^{\alpha}+\right.$ $\left.L_{2}^{\alpha}+L_{3}^{\alpha}, \mathbb{H}^{(0)}\right]=0$, with the $S L(2)$ generators $L_{k}^{\alpha}$ defined in (6.12). This allows one to classify its eigenstates $\Psi_{\boldsymbol{q}}\left(z_{i}\right)$ according to irreducible components in the tensor product of three $S L(2)$ modules, $\mathcal{V}_{j} \otimes \mathcal{V}_{j} \otimes \mathcal{V}_{j}$. Applying the relation (6.14) twice, one finds that $\Psi_{\boldsymbol{q}}\left(z_{i}\right)$ carries the total three-particle conformal spin equal to $J_{123}=3 j+N$ with $j=1$ and integer $N \geq 0$. This is the eigenvalue of the three-particle quadratic Casimir

$$
q_{2}^{(0)}=L_{12}^{2}+L_{23}^{2}+L_{31}^{2}-3 j(j-1)
$$


such that

$$
q_{2}^{(0)} \Psi_{\boldsymbol{q}}\left(z_{i}\right)=J_{123}\left(J_{123}-1\right) \Psi_{\boldsymbol{q}}\left(z_{i}\right),
$$

with $J_{123}=3+N$. On the other hand, the two-particle conformal spin in any subchannel, say (12), is not fixed anymore $2 j \leq J_{12} \leq 2 j+N$. This means that, in distinction with the $L=2$ particle operators discussed in the previous section (see Eq. (6.19)), the conformal invariance alone does not permit us to determine the eigenstates of the three-particle dilatation operator. Still, it allows us to write a general expression for the eigenstates $\Psi_{\boldsymbol{q}}\left(z_{i}\right)$ as a sum over the states possessing definite conformal spins $J_{123}=3 j+N$ and $J_{12}=2 j+n$ and reduce the eigenproblem (6.57) to finding the corresponding expansion coefficients.

The conformal symmetry also dictates that the eigenstates $\Psi_{\boldsymbol{q}}\left(z_{i}\right)$ have to be orthogonal to each other with respect to the $S L(2)$ scalar product (for a review see Refs. [46, 54]). In the momentum representation, the same condition looks like

$$
\left\langle\Psi_{\boldsymbol{q}} \mid \Psi_{\boldsymbol{q}^{\prime}}\right\rangle=\int_{0}^{1}[d u]_{3} u_{1} u_{2} u_{3} P_{\boldsymbol{q}}\left(u_{i}\right) P_{\boldsymbol{q}^{\prime}}\left(u_{i}\right)=\delta_{\boldsymbol{q} \boldsymbol{q}^{\prime}} .
$$

This relation is a counter-part of the orthogonality condition for the Gegenbauer polynomials defining the conformal polynomials for $L=2$ operators, Eq. (6.3). For the eigenstates of definite parity, the orthogonality condition looks like

$$
\left\langle\Psi_{\boldsymbol{q}}^{ \pm} \mid \Psi_{\boldsymbol{q}^{\prime}}^{ \pm}\right\rangle=\delta_{\boldsymbol{q \boldsymbol { q } ^ { \prime }}}, \quad\left\langle\Psi_{\boldsymbol{q}}^{ \pm} \mid \Psi_{\boldsymbol{q}^{\prime}}^{\mp}\right\rangle=0
$$

The dilatation operator is a self-adjoint operator with respect to the scalar product (6.68) and, as a consequence, its eigenvalues $\gamma_{\boldsymbol{q}}^{(0)}$ are real.

The eigenproblem for the one-loop dilatation operator (6.57) can be solved exactly thanks to the existence of the hidden, $S L(2)$ invariant conserved charge $q_{3}^{(0)}$

$$
\left[\mathbb{H}^{(0)}, q_{3}^{(0)}\right]=\left[L_{1}^{\alpha}+L_{2}^{\alpha}+L_{3}^{\alpha}, q_{3}^{(0)}\right]=0
$$

where we introduced a superscript to indicate that this charge could acquire higher order perturbative corrections in coupling constant. The charge $q_{3}^{(0)}$ is defined as

$$
q_{3}^{(0)}=\frac{i}{2}\left[L_{12}^{2}, L_{23}^{2}\right]=\frac{i}{2}\left[L_{31}^{2}, L_{12}^{2}\right]
$$

with the two-particle Casimir operators $L_{j k}^{2}$ given by (6.18). As a result, the Schrödinger equation (6.57) turns out to be completely integrable and the one-loop dilatation operator $\mathbb{H}^{(0)}$ can be mapped into the Hamiltonian of a completely integrable Heisenberg magnet of length $L=3$ and spins being the generators of the conformal $S L(2)$ group (for a review see Ref. 6]).

According to (6.70), the eigenstates of the dilatation operator $\mathbb{H}^{(0)}$ can be chosen to diagonalize the conserved charge

$$
q_{3}^{(0)} \Psi_{\boldsymbol{q}}\left(z_{1}, z_{2}, z_{3}\right)=q \Psi_{\boldsymbol{q}}\left(z_{1}, z_{2}, z_{3}\right)
$$

Together with the total conformal spin $J_{123}=3 j+N$, the charge $q$ define the total set of quantum numbers parameterizing the solutions to the spectral problem (6.57), $\boldsymbol{q}=(N, q)$. A distinguished feature of $q_{3}^{(0)}$ is that it is invariant under cyclic permutations and changes the sign under permutation of any pair of fields

$$
\left[\mathbb{P}, q_{3}^{(0)}\right]=\left\{\mathbb{P}_{12}, q_{3}^{(0)}\right\}=0
$$


As far as the dependence of $\mathbb{H}^{(0)}$ on the conserved charge is concerned, one finds using (6.59)

$$
\mathbb{H}^{(0)}\left(q_{3}^{(0)}\right)=\mathbb{P}_{12} \mathbb{H}^{(0)}\left(q_{3}^{(0)}\right) \mathbb{P}_{12}=\mathbb{H}^{(0)}\left(\mathbb{P}_{12} q_{3}^{(0)} \mathbb{P}_{12}\right)=\mathbb{H}^{(0)}\left(-q_{3}^{(0)}\right),
$$

that is, the eigenstates carrying opposite values of the conserved charge have the same energy

$$
\Psi_{-\boldsymbol{q}}\left(z_{i}\right)=\mathbb{P}_{12} \Psi_{\boldsymbol{q}}\left(z_{i}\right), \quad \gamma_{\boldsymbol{q}}^{(0)}=\gamma_{-\boldsymbol{q}}^{(0)},
$$

where $-\boldsymbol{q}=(N,-q)$. For $q=0$ one finds from (6.75) that $\Psi_{\boldsymbol{q}=0}\left(z_{i}\right)$ has the parity +1 and, therefore, $\Psi_{\boldsymbol{q}}^{-}\left(z_{i}\right)=0$. For $q \neq 0$, it follows from (6.72) and (6.75) that the operator $q_{3}^{(0)}$ maps into each other "degenerate" eigenstates with positive and negative parity

$$
q_{3}^{(0)} \Psi_{\boldsymbol{q}}^{ \pm}\left(z_{1}, z_{2}, z_{3}\right)=q \Psi_{\boldsymbol{q}}^{\mp}\left(z_{1}, z_{2}, z_{3}\right) .
$$

We already observed that the symmetry of the dilatation operator under permutations of fields leads to the degeneracy of eigenstates with nonvanishing quasimomentum. According to (6.75), integrability extends this property to the eigenstates with $\theta_{\boldsymbol{q}}=0$ and $q \neq 0$. The eigenstate with $q=0$ is the only one which is not paired. It has the quasimomentum $\theta_{\boldsymbol{q}=0}=0$ and only appears for even $N$.

The exact solution to the eigenproblem is given by the Bethe Ansatz technique. For given $N$, the eigenspectrum is determined by a set of $N$ real numbers $\lambda_{1}, \ldots, \lambda_{N}$, the so-called Bethe roots, satisfying the system of transcendental equations

$$
\left(\frac{\lambda_{n}+i}{\lambda_{n}-i}\right)^{3}=\prod_{k=1, k \neq n}^{N} \frac{\lambda_{n}-\lambda_{k}-i}{\lambda_{n}-\lambda_{k}+i}, \quad(n=1, \ldots, N) .
$$

The corresponding exact values of the "energy", quasimomentum and the conserved charge are given, respectively, by

$$
\gamma_{\boldsymbol{q}}^{(0)}=\sum_{k=1}^{N} \frac{2}{\lambda_{k}^{2}+1}, \quad \mathrm{e}^{i \theta \boldsymbol{q}}=\prod_{k=1}^{N} \frac{\lambda_{k}-i}{\lambda_{k}+i}, \quad q=-2 \operatorname{Im} \prod_{k=1}^{N}\left(1-\frac{i}{\lambda_{k}}\right) .
$$

We remind that cyclic symmetry of the three-particle states imposes the selection rule $\theta_{\boldsymbol{q}}=0$. The results of the calculation of the one-loop anomalous dimension $\gamma_{\boldsymbol{q}}^{(0)}$ for $0 \leq N \leq 20$ is shown in Fig. 9. Although $\gamma_{\boldsymbol{q}}^{(0)}$ can not be written in a closed form, one can work out asymptotic expressions which approximate the exact result with a good accuracy [55, 3, 4, 5].

\subsubsection{Criterium of higher loop integrability}

Integrability played a vital role in the diagonalization of the one-loop dilatation operator. It appeared atop of the conformal symmetry and simplified significantly the solution of the spectral problem (6.57) but its origin remained unclear. The question arises whether this symmetry will survive to higher loops in QCD and SYM theories. If integrability were ultimately tied to the conformal symmetry then one should expect, based on the discussion in Sect. 6.1.3 that both symmetries are broken starting from two loops in gauge theory with $\beta\left(g^{2}\right) \neq 0$. As we will argue below this does not happen and integrability survives the conformal symmetry breaking.

To two-loop order, the dilatation operator takes the form $\mathbb{H}=\lambda \mathbb{H}^{(0)}+\lambda^{2} \mathbb{H}^{(1)}+\mathcal{O}\left(\lambda^{3}\right)$, Eq. (3.2) with the two-loop correction given in the momentum representation by (5.46). The 
eigenspectrum of $\mathbb{H}^{(0)}$ was determined in the previous section. Thinking about $\mathbb{H}$ as a quantum mechanical Hamiltonian, one can apply the perturbation theory to calculate the corrections to the eigenspectrum of the two-loop dilatation operator induced by the "perturbation" $\mathbb{H}^{(1)}$. In spite of the fact that $\mathbb{H}$ does not respect the conformal symmetry, the eigenfunctions of the one-loop dilatation operator provide a complete set of functions endowed with the scalar product (6.68). In other words, the eigenstates of the all-loop dilatation operator $\mathbb{H}$ can be decomposed over the leading order eigenstates $\Psi_{\boldsymbol{q}}\left(z_{i}\right)$. Since $\mathbb{H}$ has the same symmetry under cyclic permutations of particles as $\mathbb{H}^{(0)}$, this decomposition involves the states with the same quasimomentum $\theta_{\boldsymbol{q}}$. As before, the eigenstates of $\mathbb{H}$ with $\theta_{\boldsymbol{q}} \neq 0$ are double-degenerate while for $\theta_{\boldsymbol{q}}=0$ the degeneracy can be lifted in general.

Applying the conventional perturbation theory we find that the eigenstate of $\mathbb{H}$ is given by

$$
\Psi_{\boldsymbol{q}}^{(1)}\left(z_{i}\right)=\Psi_{\boldsymbol{q}}\left(z_{i}\right)+\lambda \sum_{\boldsymbol{q}^{\prime} \neq \boldsymbol{q}} \frac{\left\langle\Psi_{\boldsymbol{q}^{\prime}}\left|\mathbb{H}^{(1)}\right| \Psi_{\boldsymbol{q}}\right\rangle}{\gamma_{\boldsymbol{q}}^{(0)}-\gamma_{\boldsymbol{q}^{\prime}}^{(0)}} \Psi_{\boldsymbol{q}^{\prime}}\left(z_{i}\right)+\mathcal{O}\left(\lambda^{2}\right)
$$

However, due to degeneracy of the spectrum (6.75), the expansion is not well-defined for $\boldsymbol{q}^{\prime}=-\boldsymbol{q}$. We recall that the paired eigenstates have opposite parity, $\Psi_{\boldsymbol{q}}^{ \pm}\left(z_{i}\right)$, Eq. (6.63). The perturbation $\mathbb{H}^{(1)}$ preserves the parity, $\left[\mathbb{H}^{(1)}, \mathbb{P}_{12}\right]=0$, and, therefore, the eigenstates with positive and negative parity get decoupled from each other

$$
\left\langle\Psi_{\boldsymbol{q}^{\prime}}^{ \pm}\left|\mathbb{H}^{(1)}\right| \Psi_{\boldsymbol{q}}^{\mp}\right\rangle=0
$$

Then, in the sector with a definite parity, the energy levels of $\mathbb{H}^{(0)}$ are not degenerate and the conventional formulas are at work. In particular, the two-loop correction to the eigenvalue of the dilatation operator with positive/negative parity is given by

$$
\gamma_{\boldsymbol{q}}^{(1), \pm}=\left\langle\Psi_{\boldsymbol{q}}^{ \pm}\left|\mathbb{H}^{(1)}\right| \Psi_{\boldsymbol{q}}^{ \pm}\right\rangle
$$

with the normalization condition (6.69). Substituting $\Psi_{\boldsymbol{q}}^{ \pm}\left(z_{i}\right)$ by their expressions (6.63) the matrix element can be evaluated as

$$
\left\langle\Psi_{\boldsymbol{q}}^{ \pm}\left|\mathbb{H}^{(1)}\right| \Psi_{\boldsymbol{q}}^{ \pm}\right\rangle=\left\langle\Psi_{\boldsymbol{q}}\left|\mathbb{H}^{(1)}\right| \Psi_{\boldsymbol{q}}\right\rangle \pm\left\langle\Psi_{\boldsymbol{q}}\left|\mathbb{H}^{(1)}\right| \Psi_{-\boldsymbol{q}}\right\rangle
$$

where we took into account that $\left\langle\Psi_{-\boldsymbol{q}}\left|\mathbb{H}^{(1)}\right| \Psi_{-\boldsymbol{q}}\right\rangle=\left\langle\Psi_{\boldsymbol{q}}\left|\mathbb{P}_{12} \mathbb{H}^{(1)} \mathbb{P}_{12}\right| \Psi_{\boldsymbol{q}}\right\rangle=\left\langle\Psi_{\boldsymbol{q}}\left|\mathbb{H}^{(1)}\right| \Psi_{\boldsymbol{q}}\right\rangle$. Thus, the two-loop correction to the dilatation operator lifts the leading order degeneracy, $\gamma_{\boldsymbol{q}}^{(0), \pm}=\gamma_{\boldsymbol{q}}^{(0)}$, Eq. (6.65),

$$
\gamma_{\boldsymbol{q}}^{(1),+}-\gamma_{\boldsymbol{q}}^{(1),-}=2\left\langle\Psi_{\boldsymbol{q}}\left|\mathbb{H}^{(1)}\right| \Psi_{-\boldsymbol{q}}\right\rangle .
$$

We expect that $\gamma_{\boldsymbol{q}}^{(1),+}=\gamma_{\boldsymbol{q}}^{(1),-}$ for the eigenstates with $\theta_{\boldsymbol{q}} \neq 0$. Indeed, examining the identity $\left\langle\Psi_{\boldsymbol{q}}\left|\left[\mathbb{P}, \mathbb{H}^{(1)}\right]\right| \Psi_{-\boldsymbol{q}}\right\rangle=0$ it is easy to see that the matrix element in the right-hand side of (6.83) vanishes for $\theta_{\boldsymbol{q}} \neq 0$. Thus, the two-loop correction to the dilatation operator could only affect the pairing of the eigenstates with the quasimomentum $\theta_{\boldsymbol{q}}=0$.

The necessary and sufficient condition for pairing to persist to two loops is (for $q \neq 0$ )

$$
\left\langle\Psi_{\boldsymbol{q}}\left|\mathbb{H}^{(1)}\right| \Psi_{-\boldsymbol{q}}\right\rangle=0
$$

It turns out that this relation implies the existence of the conserved charge

$$
q_{3}=q_{3}^{(0)}+\lambda q_{3}^{(1)}+\mathcal{O}\left(\lambda^{2}\right),
$$


which has the same discrete symmetry as the leading order charge, $\left[\mathbb{P}, q_{3}\right]=\left\{\mathbb{P}_{12}, q_{3}\right\}=0$ and commutes with the dilatation operator $\left[q_{3}, \mathbb{H}\right]=0$. When expanded in powers of $\lambda$, the last relation reads

$$
\left[q_{3}^{(0)}, \mathbb{H}^{(1)}\right]+\left[q_{3}^{(1)}, \mathbb{H}^{(0)}\right]=0 .
$$

Going over to the matrix elements one obtains

$$
\left(q-q^{\prime}\right)\left\langle\Psi_{\boldsymbol{q}}\left|\mathbb{H}^{(1)}\right| \Psi_{\boldsymbol{q}^{\prime}}\right\rangle=\left(\gamma_{\boldsymbol{q}}^{(0)}-\gamma_{\boldsymbol{q}^{\prime}}^{(0)}\right)\left\langle\Psi_{\boldsymbol{q}}\left|q_{3}^{(1)}\right| \Psi_{\boldsymbol{q}^{\prime}}\right\rangle
$$

One puts $q^{\prime}=-q$, applies (6.75) and arrives at (6.84). Eq. (6.87) can be used to determine the matrix elements $\left\langle\Psi_{\boldsymbol{q}}\left|q_{3}^{(1)}\right| \Psi_{\boldsymbol{q}^{\prime}}\right\rangle$ for $q \neq \pm q^{\prime}$. For $q= \pm q^{\prime}$ both sides of the relation (6.87) vanish simultaneously. The reason for this is that (6.86) defines the charge $q_{3}^{(1)}$ up to an addition of an operator commuting with the one-loop dilatation operator. This ambiguity can be fixed by imposing additional conditions on $q_{3}^{(1)}$. For instance, we will show below that $q_{3}^{(1)}$ can be defined in such a way that the two-loop charge (6.85) has the same eigenvalues as the one-loop charge while its eigenstates are modified by $\sim \lambda$ corrections.

Let us examine expressions for the eigenstates of two-loop dilatation operator

$$
\Psi_{\boldsymbol{q}}^{(1), \pm}\left(z_{i}\right)=\Psi_{\boldsymbol{q}}^{ \pm}\left(z_{i}\right)+\lambda \sum_{q^{\prime} \neq q} \frac{\left\langle\Psi_{\boldsymbol{q}^{\prime}}^{ \pm}\left|\mathbb{H}^{(1)}\right| \Psi_{\boldsymbol{q}}^{ \pm}\right\rangle}{\gamma_{\boldsymbol{q}}^{(0)}-\gamma_{\boldsymbol{q}^{\prime}}^{(0)}} \Psi_{\boldsymbol{q}^{\prime}}^{ \pm}\left(z_{i}\right) \equiv(\mathbb{1}+\lambda \mathbb{Z}) \Psi_{\boldsymbol{q}}^{ \pm}\left(z_{i}\right)
$$

where in distinction with (6.79) the sum in the right-hand side is well-defined for all $q^{\prime}$. Here the notation was introduced for the operator $\mathbb{Z}$

$$
\mathbb{Z}\left|\Psi_{\boldsymbol{q}}^{ \pm}\right\rangle=\sum_{q^{\prime} \neq q} \frac{\left\langle\Psi_{\boldsymbol{q}^{\prime}}^{ \pm}\left|\mathbb{H}^{(1)}\right| \Psi_{\boldsymbol{q}}^{ \pm}\right\rangle}{\gamma_{\boldsymbol{q}}^{(0)}-\gamma_{\boldsymbol{q}^{\prime}}^{(0)}}\left|\Psi_{\boldsymbol{q}^{\prime}}^{ \pm}\right\rangle
$$

It is easy to verify that this operator is antihermitian, $(\mathbb{Z})^{\dagger}=-\mathbb{Z}$.

The operator $(\mathbb{1}+\lambda \mathbb{Z})$ has a simple physical meaning - it rotates the one-loop eigenstates into the two-loop ones. Let us perform the following unitary transformation of the two-loop Hamiltonian

$$
\widetilde{\mathbb{H}}=(\mathbb{1}-\lambda \mathbb{Z}) \mathbb{H}(\mathbb{1}+\lambda \mathbb{Z})=\mathbb{H}^{(0)}+\lambda\left(\mathbb{H}^{(1)}+\left[\mathbb{H}^{(0)}, \mathbb{Z}\right]\right)+\mathcal{O}\left(\lambda^{2}\right)
$$

By construction, $\widetilde{\mathbb{H}}$ has the same eigenvalues as the two-loop Hamiltonian $\mathbb{H}$ whereas the corresponding eigenstates coincide with the one-loop eigenstates $\widetilde{\mathbb{H}}\left|\Psi_{\boldsymbol{q}}^{ \pm}\right\rangle=\gamma_{\boldsymbol{q}}^{ \pm}\left|\Psi_{\boldsymbol{q}}^{ \pm}\right\rangle$with $\gamma_{\boldsymbol{q}}^{ \pm}=$ $\gamma_{\boldsymbol{q}}^{(0)}+\lambda \gamma_{\boldsymbol{q}}^{(1), \pm}+\mathcal{O}\left(\lambda^{2}\right)$. Combining this relation together with (6.75) one obtains

$$
\left(\widetilde{\mathbb{H}} q_{3}^{(0)}-q_{3}^{(0)} \widetilde{\mathbb{H}}\right)\left|\Psi_{\boldsymbol{q}}^{\mp}\right\rangle=q\left(\gamma_{\boldsymbol{q}}^{ \pm}-\gamma_{\boldsymbol{q}}^{\mp}\right)\left|\Psi_{\boldsymbol{q}}^{ \pm}\right\rangle=\left[\lambda q\left(\gamma_{\boldsymbol{q}}^{(1), \pm}-\gamma_{\boldsymbol{q}}^{(1), \mp}\right)+\mathcal{O}\left(\lambda^{2}\right)\right]\left|\Psi_{\boldsymbol{q}}^{ \pm}\right\rangle .
$$

If the degeneracy of eigenvalues survives to two loops, $q\left(\gamma_{\boldsymbol{q}}^{(1), \pm}-\gamma_{\boldsymbol{q}}^{(1), \mp}\right)=0$, then the operators $\widetilde{\mathbb{H}}$ and $q_{3}^{(0)}$ commute leading to

$$
\left[\widetilde{\mathbb{H}}, q_{3}^{(0)}\right]=\left[\mathbb{H},(1+\lambda \mathbb{Z}) q_{3}^{(0)}(1-\lambda \mathbb{Z})\right]=0+\mathcal{O}\left(\lambda^{2}\right)
$$

We deduce from this relation that the pairing of eigenvalues allows us to construct the operator satisfying (6.86) and (6.85)

$$
q_{3}=q_{3}^{(0)}-\lambda\left[q_{3}^{(0)}, \mathbb{Z}\right]+\mathcal{O}\left(\lambda^{2}\right)
$$


Taking into account (6.89) and (6.76), it is straightforward to verify that the operator $q_{3}^{(1)}=$ $-\left[q_{3}^{(0)}, \mathbb{Z}\right]$ satisfies (6.87) and has the same discrete symmetries, Eq. (6.73), as the leading term $q_{3}^{(0)}$. By construction, the operator $q_{3}$ commutes (up to higher order corrections) with the twoloop Hamiltonian. Since $q_{3}$ is obtained from $q_{3}^{(0)}$ by the same unitary transformation as in (6.90), its eigenvalues do not receive perturbative corrections while the eigenstates are given by (6.88)

$$
q_{3}\left|\Psi_{\boldsymbol{q}}^{(1)}\right\rangle=\left[q+\mathcal{O}\left(\lambda^{2}\right)\right]\left|\Psi_{\boldsymbol{q}}^{(1)}\right\rangle
$$

We recall that the definition of $q_{3}$ is ambiguous since one can fulfill (6.86) by adding to $q_{3}^{(1)}$ an arbitrary function of $q_{3}^{(0)}$. Eq. (6.94) corresponds to a particular choice of the 'gauge' in which eigenvalues of $q_{3}$ do not receive radiative corrections to two loops.

In an analogous manner one finds the higher order analogue $q_{2}$ of the quadratic conformal Casimir $q_{2}^{(0)}$, Eq. (6.66). It is determined by the same rotation matrix $\mathbb{Z}$ such that $q_{2}=$ $q_{2}^{(0)}-\lambda\left[q_{2}^{(0)}, \mathbb{Z}\right]+\mathcal{O}\left(\lambda^{2}\right)$. Notice however that the charge $q_{2}$, having an opposite permutation symmetry compared to $q_{3}$, does not require the pairing of eigenvalues with opposite parity for its commutativity with the Hamiltonian $\mathbb{H}$ to hold. As a consequence of our construction, we found a unitary transformation which gives the two-loop Hamiltonian $\widetilde{\mathbb{H}}$ which can be diagonalized simultaneously with mutually commuting leading order charges $q_{2}^{(0)}$ and $q_{3}^{(0)}$.

We would like to stress that existence of $q_{3}$ is tied solely to degeneracy of the eigenvalues of the two-loop Hamiltonian $\mathbb{H}$. The same relation works in the opposite direction. If the Hamiltonian $\mathbb{H}$ possesses the conserved charge $q_{3}$ which is odd under permutation of particles then the degeneracy of the eigenspectrum follows in the same manner as at the leading order, Eq. (6.74).

The commutativity of the rotated two-loop Hamiltonian $\widetilde{\mathbb{H}}$ with the quadratic Casimir $q_{2}^{(0)}$ does not imply the restoration of the conformal symmetry. The persistence of the latter would require that the local Wilson operators $\mathcal{O}_{\boldsymbol{q}}(0)$, Eq. (3.4), corresponding to the eigenstates (6.88) have to satisfy the relations similar to (6.38), that is $\left[\mathcal{O}_{\boldsymbol{q}}(0), \mathbb{L}^{+}\right]=0$ and $\left[\mathcal{O}_{\boldsymbol{q}}(0), \mathbb{L}^{0}\right] \sim \mathcal{O}_{\boldsymbol{q}}(0)$. Notice that $\mathbb{H}$ defines radiative corrections to $\mathbb{L}^{0}$ and the second relation is indeed satisfied in the basis (6.88). The question thus boils down to the study of $\mathbb{L}^{+}$. A simple consideration demonstrates that the higher order corrections in theories with nonvanishing $\beta$-function lead to $\left[\mathcal{O}_{\boldsymbol{q}}(0), \mathbb{L}^{+}\right] \sim \beta\left(g^{2}\right)$ (see, e.g., [46, 32]). For the sake of the argument let us discuss the well-studied twist-two sector, which was explored in detail in Sect. 6.1.3. As we have shown there, for $\beta=0$, one can bring the Ward identities for both $\mathbb{L}^{0}$, Eq. (6.27), and $\mathbb{L}_{+}$, Eq. (6.28), to the needed form (6.38) by going to the $\overline{\mathrm{CS}}$ scheme with the transformation matrix $\mathcal{B}$, (6.36) and (6.37), respectively. Thus, the rotated operator $\widehat{\mathcal{O}}_{n n}$ verifies (6.38) and defines the lowest weight vector of the irreducible representation of the conformal group. However, in case when the $\beta$-function is nonzero, while the $\mathbb{L}^{0}$ operator can still be diagonalized with the rotation

matrix $\mathcal{B}$, the commutator of the conformal boost $\mathbb{L}^{+}$with the rotated Wilson operator $\widehat{\mathcal{O}}_{n n}$ is proportional to $\beta\left(g^{2}\right)$ and, therefore, $\widehat{\mathcal{O}}_{n n}$ does not represent the lowest weight. The same phenomenon re-occurs for three-particle operators - while one is able to diagonalize $\mathbb{L}^{0}$, with $\mathbb{Z}$, it is not the case for $\mathbb{L}^{+}$unless $\beta=0$.

\subsubsection{Numerical diagonalization of the two-loop dilatation operator}

We argued in the previous section that the degeneracy of the eigenvalues of the two-loop dilatation operator could serve as a criterium for two-loop integrability. Let us now use the expressions for 
the two-loop dilatation operators obtained in Sect. 5 to evaluate their eigenspectrum and verify whether the pairing of the eigenvalues present at one-loop in QCD and SYM theories survives to two loops.

We recall that the two-loop evolution kernels have been evaluated in the momentum representation. In this representation the eigenvalue problem looks like

$$
\int[d u]_{3} \mathbb{V}(\boldsymbol{u} \mid \boldsymbol{v}) P_{\boldsymbol{q}}(\boldsymbol{u})=-\gamma_{\boldsymbol{q}}(\lambda) P_{\boldsymbol{q}}(\boldsymbol{v})
$$

where the integration measure was defined in Eq. (3.19), while the integration region over the $u$-variables is determined by the support properties of the evolution kernel $\mathbb{V}(\boldsymbol{u} \mid \boldsymbol{v})$. Notice that the eigenvalues $\gamma_{\boldsymbol{q}}(\lambda)$ do not depend on the "external" momenta $\boldsymbol{v}=\left(v_{1}, v_{2}, v_{3}\right)$. One can explore this fact to simplify the actual calculation of the anomalous dimensions. Indeed, the calculation of the two-loop evolution kernel has been performed in Sect. 5 in the kinematics $0 \leq v_{1}, v_{2}, v_{3} \leq 1$ and $v_{1}+v_{2}+v_{3}=1$. In this case, the phase space for integrated $u$-momenta is the one shown in Fig. 3 .

As explained in Sec. 6.1.3 for two-particle operators, the conformal symmetry is broken beyond leading order in gauge coupling and so the eigenfunctions, depending on the quantum number $N$, of the dilatation operator are changed by $\mathcal{O}(\lambda)$ corrections. This symmetry breaking is induced by the renormalization prescription of the composite operators. In fact the breakdown of special conformal symmetry arises already at one-loop level and this determines via the conformal constraint (6.30) the eigenfunctions of the two-loop dilatation operator. Hence, one can get rid of this conformal symmetry breaking at this order by means of a finite renormalization of the operator to one-loop accuracy. It can be shown in general that the known special conformal anomaly in the two-particle subchannels to one-loop accuracy together with the conformal constraint, similar to Eq. (6.30), is consistent with our result (5.46).

For the $\beta(g)=0$ case, the finite renormalization alluded to above yields the anomalous dimension matrix which is identical to the one found by means of taking the forward limit. Therefore, the simplest way to calculate the eigenvalues of the two-loop evolution kernel within the conformal subtraction scheme is to take the forward limit of the kernel, i.e., impose the following condition on the light-cone particle momenta

$$
u_{1}+u_{2}+u_{3}=v_{1}+v_{2}+v_{3}=0 .
$$

As was already explained in Sect.6.1.5. the forward limit effectively corresponds to taking forward matrix elements of three-particle operators. This does not change the eigenspectrum of the dilatation operator but drastically simplifies the numerical calculation of moments since the mixing with the operators containing total derivatives is automatically removed.

In the forward limit, the evolution kernel looks differently as compared to the one in the off-forward kinematics $\sum_{n} v_{n}=1$. It turns out that the two kernels are related to each other through the following limiting procedure [36]. Let us set

$$
u_{i}=\frac{x_{i}}{\tau}, \quad v_{i}=\frac{y_{i}}{\tau}, \quad x_{1}+x_{2}+x_{3}=y_{1}+y_{2}+y_{3}=\tau
$$

and take the limit $\tau \rightarrow 0$. Then, the kernel in the forward limit reads

$$
\mathbb{P}(\boldsymbol{x} \mid \boldsymbol{y})=\operatorname{LIM} \mathbb{V}(\boldsymbol{u} \mid \boldsymbol{v}) \equiv \lim _{\tau \rightarrow 0} \frac{1}{\tau^{2}} \mathbb{V}\left(\frac{x_{1}}{\tau}, \frac{\tau-x_{1}-x_{3}}{\tau}, \frac{x_{3}}{\tau} \mid \frac{y_{1}}{\tau}, \frac{\tau-y_{1}-y_{3}}{\tau}, \frac{y_{3}}{\tau}\right),
$$


where $\boldsymbol{x}$ denotes now the variables $x_{1}$ and $x_{3}$ (with $x_{2}=-x_{1}-x_{3}$ ) and similarly for $\boldsymbol{y}$. Before the limit (6.98) can be performed, the support of the kernel must be extended. This extension procedure is described in Appendix A. Performing the limiting procedure (6.98) in both sides of the evolution equation (6.95) one obtains the eigenproblem in the forward limit in the following form

$$
\int d \boldsymbol{x} \mathbb{P}(\boldsymbol{x} \mid \boldsymbol{y}) C_{\boldsymbol{q}}\left(x_{1}, x_{3}\right)=-\gamma_{\boldsymbol{q}}(\lambda) C_{\boldsymbol{q}}\left(y_{1}, y_{3}\right)
$$

with $d \boldsymbol{x}=d x_{1} d x_{3}$. Here the eigenvalues $\gamma_{\boldsymbol{q}}(\lambda)$ are the same as in (6.95) while the eigenfunctions are related as

$$
C_{\boldsymbol{q}}\left(y_{1}, y_{3}\right)=\lim _{\tau \rightarrow 0} \tau^{N} P_{\boldsymbol{q}}\left(\frac{y_{1}}{\tau}, \frac{\tau-y_{1}-y_{3}}{\tau}, \frac{y_{3}}{\tau}\right)=P_{\boldsymbol{q}}\left(y_{1},-y_{1}-y_{3}, y_{3}\right) .
$$

In the last relation we used the fact that the eigenstates of the evolution kernel are homogenous polynomials in momentum fractions of degree $N \geq 0$. Obviously, the polynomials $C_{\boldsymbol{q}}\left(y_{1}, y_{3}\right)$ have the same property. They are uniquely specified by the set of coefficients in the expansion of $C_{\boldsymbol{q}}\left(y_{1}, y_{3}\right)$ over the basis $y_{1}^{k} y_{3}^{N-k}$ (with $\left.k=0, \ldots, N\right)$. The total number of these coefficients is $N+1$ and it matches the total number of three-particle operators with $N$ derivatives having nonvanishing matrix elements in the forward limit, i.e., free from total derivatives. In the basis $y_{1}^{k} y_{3}^{N-k}$ the forward evolution kernel $\mathbb{P}(\boldsymbol{x} \mid \boldsymbol{y})$ is represented by a finite dimensional mixing matrix

$$
\int d \boldsymbol{x} \mathbb{P}(\boldsymbol{x} \mid \boldsymbol{y}) x_{1}^{N-n} x_{3}^{n}=\sum_{m=0}^{N} \Lambda_{n k}(\lambda) y_{1}^{N-k} y_{3}^{k},
$$

whose entries depend both on $N$ and the gauge coupling $\lambda$. Then, to find the spectrum of the anomalous dimensions it suffices to solve the characteristic equation for the mixing matrix for each value of $N \geq 0$

$$
\operatorname{det}\left(\Lambda-\gamma_{\boldsymbol{q}}(\lambda)\right)=0 .
$$

Evaluation of the two-loop mixing matrix in the forward limit is a straightforward but tedious exercise. For given $N$, the mixing matrix has dimension $N+1$ and, therefore, the characteristic equation has $N+1$ solutions for $\gamma_{\boldsymbol{q}}$. All eigenvalues of the mixing matrix are real. This property is a consequence of the conformal invariance of the dilatation operator to one-loop order. In addition, some of the eigenstates of the mixing matrix are degenerate. To one-loop order all eigenstates except the one with $q=0$ are paired and the eigenspectrum contains $[1+N / 2]$ different energy levels. To two-loop order, the pairing can be only lifted for the eigenstates with vanishing quasimomentum. We remind that the cyclic symmetry of Wilson operators selects the latter eigenstates.

To begin with, one performs numerical diagonalization of the one-loop mixing matrix in QCD and SYM theories (see Fig. 9 (a)). In both cases, the one-loop dilatation operator involves the same two-particle kernel and, therefore, the one-loop mixing matrices are related to each other. One verifies that, in agreement with the exact results based on the Algebraic Bethe Ansatz, Eqs. (6.78) and (6.77), the one-loop eigenvalues coincide with the energy levels of the Heisenberg spin magnet. For given total conformal spin $J_{123}=3 j+N$, there are $N+1$ eigenstates. For $N$ odd, all energy levels are double degenerate while for $N$ even this is true for all levels except the lowest lying one. The latter eigenstate carries the conserved charge $q=0$ and carries 

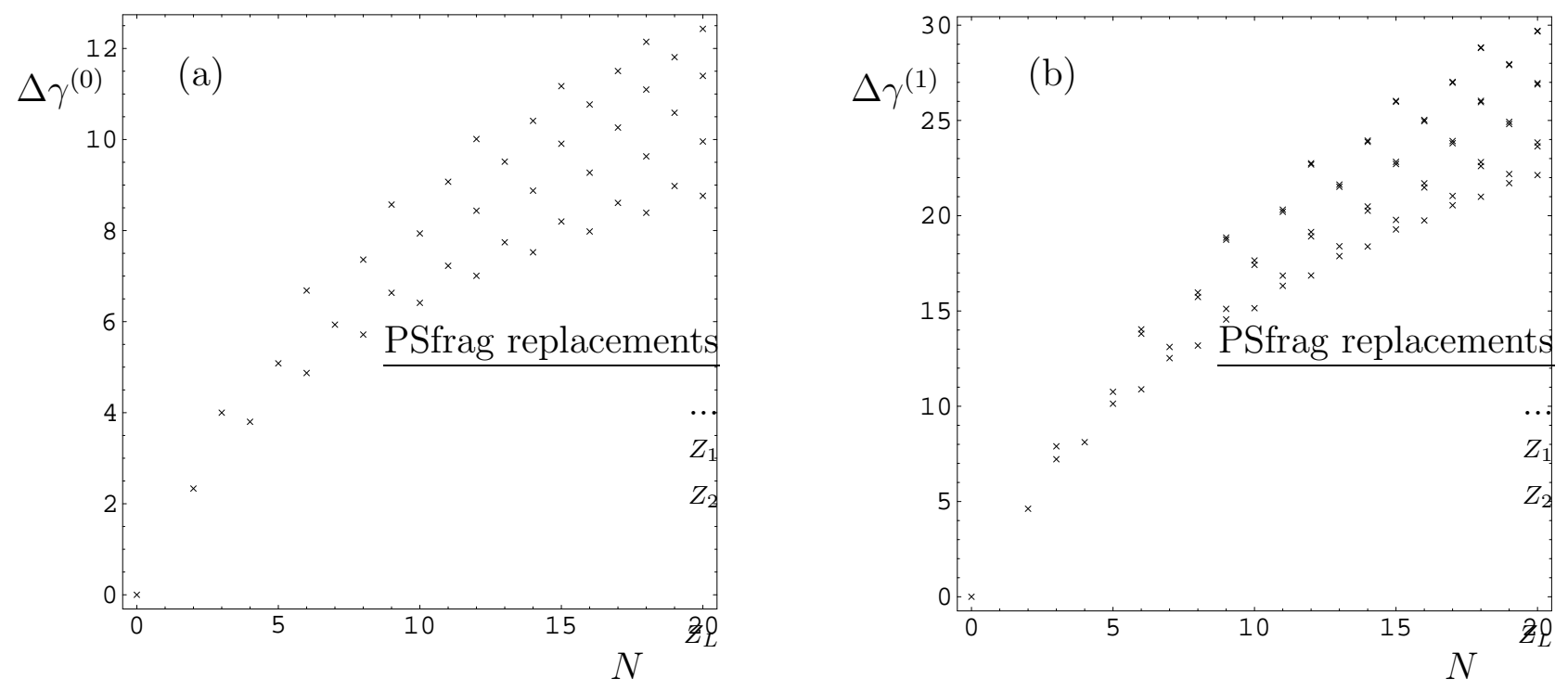

Figure 9: The spectrum at one-loop order (a) and two-loop order (b) in QCD in the $\overline{\mathrm{MS}}$ scheme. The eigenvalues with $\theta_{\boldsymbol{q}} \neq 0$ are removed from the spectra for better comparison with SYM cases where only zero quasimomentum states are physical.

the vanishing quasimomentum $\theta_{\boldsymbol{q}}=0$. For eigenstates with quasimomentum $\theta_{\boldsymbol{q}}= \pm \frac{2}{3} \pi$ the degeneracy is a consequence of the symmetry of the evolution kernel under discrete symmetries. The double degeneracy of eigenvalues with zero quasimomentum implies integrability of the oneloop Schrödinger equation (6.57). For a given $N$, the total number $m$ of the eigenstates with $\theta_{\boldsymbol{q}}=0$ is

$$
m=\frac{1}{3}(N-1)+\frac{2}{3} \bmod (N-1,3) .
$$

Among them, as we said above, there is one eigenstate with $q=0$ for even $N=2 k$ with $k=0,1,2, \ldots$, while the rest are paired.

To two-loop order, the dilatation operators in QCD and SYM theories are given by two different expressions and, therefore, the corresponding two-loop mixing matrices are not related to each other in a simple manner. Diagonalizing the latter in the forward limit, we observe the following remarkable phenomenon (see Fig. 9] (b) and Figs. 10 (b,c,d)):

- In QCD, for the baryon operator $\mathbb{O}_{\text {fun }}\left(z_{1}, z_{2}, z_{3}\right)$, the pairing is lifted for the eigenvalues with zero quasimomentum but indeed it persists for the eigenvalues with $\theta_{\boldsymbol{q}}= \pm \frac{2}{3} \pi$.

- In SYM theories with arbitrary number of supercharges $\mathcal{N}$, the one-loop pairing of eigenvalues carries on to two loops for the operator $\mathbb{O}_{\text {adj }}\left(z_{1}, z_{2}, z_{3}\right)$;

We recall that degeneracy of the eigenstates with nonzero quasimomentum is not related to integrability.

Presenting the two-loop expressions for the eigenvalues it proves convenient to introduce

$$
\Delta \gamma_{\boldsymbol{q}}^{ \pm}(\lambda) \equiv \gamma_{\boldsymbol{q}}^{ \pm}(\lambda)-3 \Gamma(\lambda)
$$

where $3 \Gamma(\lambda)$ is the anomalous dimension of the local three-fermion operator without derivatives, $N=0$. The anomalous dimension defined in such a way satisfies the normalization condition 
$\Delta \gamma_{\boldsymbol{q}}^{ \pm}(\lambda)=0$ for $N=0$. We recall that the anomalous dimension $\Gamma(\lambda)$ has the perturbative expansion (3.15) with one- and two-loop corrections in the $\overline{\mathrm{MS}}$ scheme defined in (4.12), (5.44) and (5.45). For SYM theories, we use the $\overline{\mathrm{DR}}$ scheme with $\Gamma_{\mathrm{SYM}}^{(1), \overline{\mathrm{DR}}}$ related to (5.45) as

$$
\Gamma_{\mathrm{SYM}}^{(1), \overline{\mathrm{DR}}}=\Gamma_{\mathrm{SYM}}^{(1)}-\frac{1}{6} \Gamma_{\mathrm{SYM}}^{(0)}+\frac{1}{4}(4-\mathcal{N})=2-\frac{1}{2} \mathcal{N}(\mathcal{N}-1) .
$$

The exact spectrum of eigenvalues $\Delta \gamma_{\boldsymbol{q}}^{ \pm}(\lambda)$ up to conformal spin $N=20$ is displayed in Figs. 9 and 10. They clearly demonstrate the lifting of the degeneracy in QCD for the baryon operator (2.20) compared to $\mathcal{N}$-extended SYM where it persists for all eigenstates.

The first non-trial example of the pairing phenomenon arises for $N=3$ and demonstrates the main trend, recurring for higher $N^{\text {th }}$. For $N=3$ there are four different eigenstates. Two of them have the quasimomentum $\theta_{\boldsymbol{q}}=0$ while for the remaining two possess the quasimomentum equal $\theta_{\boldsymbol{q}}= \pm 2 \pi / 3$. As was already explained, the latter eigenstates are degenerate to two loops but the corresponding Wilson operators are not cyclically symmetric. The pair of the eigenstates with $\theta_{\boldsymbol{q}}=0$ is degenerate in SYM theories for arbitrary $\mathcal{N}$ while in QCD the degeneracy is lifted to two loops.

To illustrate the phenomenon, we first present the explicit expressions for the anomalous dimensions for $N=3$ in the $\mathcal{N}=1 \mathrm{SYM}$ theory in the $\overline{\mathrm{DR}}$ scheme $^{9}$

$$
\begin{aligned}
& \Delta \gamma_{\mathrm{I}}^{ \pm}(\lambda)=\lambda 4+\lambda^{2}\left[-\frac{11}{3}+\frac{29}{24} \frac{\beta_{0}}{C_{R}}\right], \\
& \Delta \gamma_{\mathrm{II}}^{ \pm}(\lambda)=\lambda \frac{13}{4}+\lambda^{2}\left[-\frac{465}{128}+\frac{199}{192} \frac{\beta_{0}}{C_{R}}\right],
\end{aligned}
$$

where $\beta_{0} / C_{R}=6$, and in $\mathrm{QCD}$ in the $\overline{\mathrm{MS}}$ scheme

$$
\begin{aligned}
& \Delta \gamma_{\mathrm{I}}^{+}(\lambda)=\lambda 4+\lambda^{2}\left[-\frac{101}{12}+\frac{29}{24} \frac{\beta_{0}}{C_{R}}\right], \\
& \Delta \gamma_{\mathrm{I}}^{-}(\lambda)=\lambda 4+\lambda^{2}\left[-\frac{291}{32}+\frac{29}{24} \frac{\beta_{0}}{C_{R}}\right], \\
& \Delta \gamma_{\mathrm{II}}^{ \pm}(\lambda)=\lambda \frac{13}{4}+\lambda^{2}\left[-\frac{721}{96}+\frac{199}{192} \frac{\beta_{0}}{C_{R}}\right],
\end{aligned}
$$

with $\beta_{0} / C_{R}=\frac{33}{2}-N_{f}$. Here $\Delta \gamma_{\mathrm{I}}^{ \pm}(\lambda)$ and $\Delta \gamma_{\text {II }}^{ \pm}(\lambda)$ correspond to the eigenstates with $\theta_{\boldsymbol{q}}=0$ and $\theta_{\boldsymbol{q}}= \pm 2 \pi / 3$, respectively. Thus, for quarks in the fundamental representation, the two eigenstates with $\theta_{\boldsymbol{q}}=0$ have different eigenvalues starting from two loops, $\Delta \gamma_{\mathrm{I}}^{+} \neq \Delta \gamma_{\mathrm{I}}^{-}$. We remind that in QCD the three-quark baryon operator only exists for $N_{c}=3$, so that large- $N_{c}$ counting is unavailable and the non-planar diagram in Fig. 团(b) contributes and partially leads to breaking of integrability in QCD at two loops. One immediately sees from the above equations that though the degeneracy is generally lifted in QCD, the $\beta_{0}$-terms of the would-be-paired eigenvalues do coincide. In SYM they do not break integrability either. The fact that $\beta_{0}$-terms preserve degeneracy of the eigenstates implies that the two-loop integrability is not related to conformal symmetry. We shall return to this issue in Sect. 6.2.5.

\footnotetext{
${ }^{9}$ In the $\overline{\mathrm{MS}}$ scheme these anomalous dimensions coincide with Eq. (17) of Ref. [19. Though, the anomalous dimensions $\Delta \gamma_{\mathrm{II}}^{ \pm}$are not "physical" for cyclically symmetric operators, we present them to demonstrate that the degeneracy is always present for eigenvalues with nonzero quasimomentum.
} 

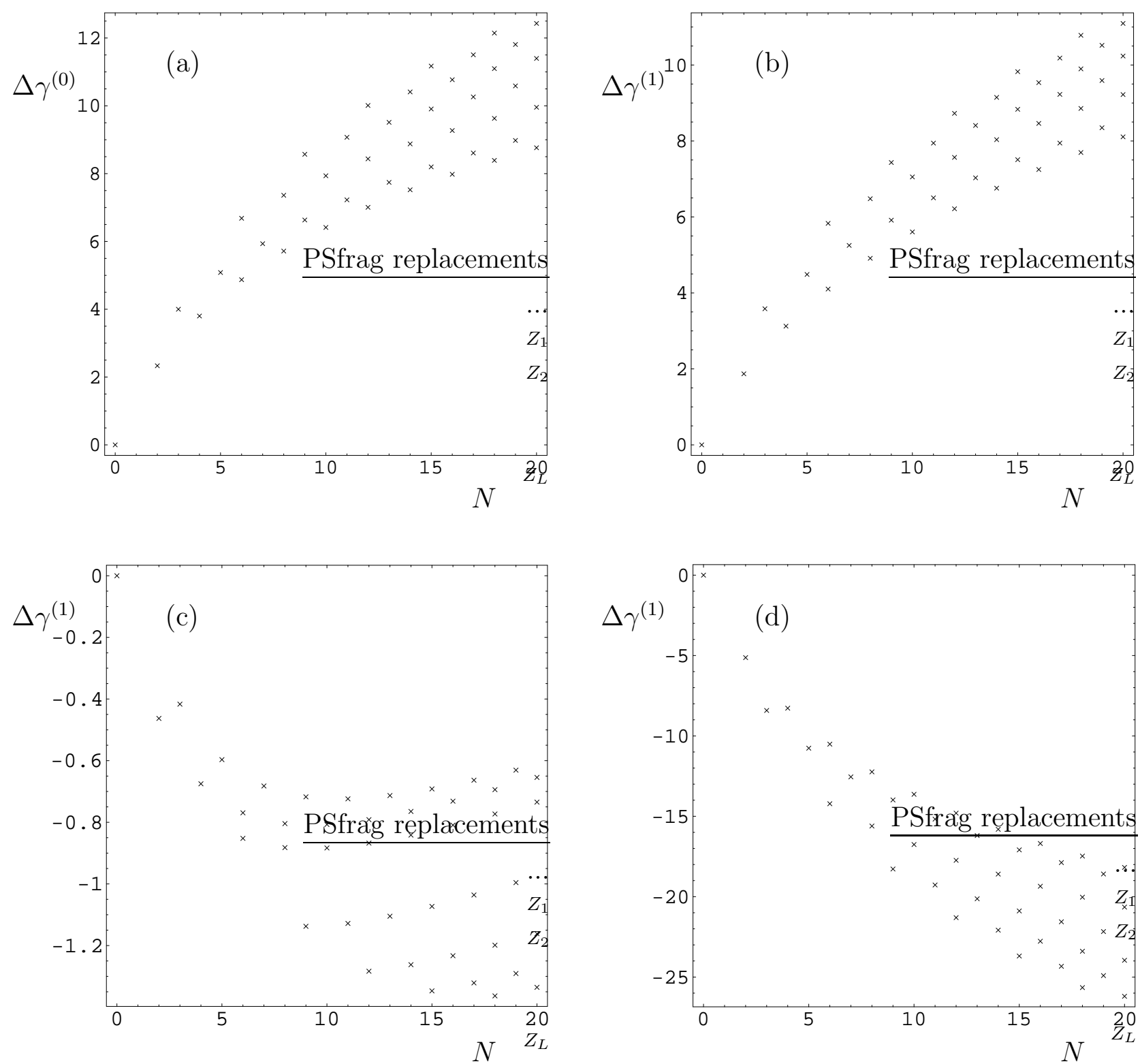

Figure 10: The spectrum at one-loop order (a) and two-loop order in SYM theories in the $\overline{\mathrm{DR}}$ scheme for $\mathcal{N}=1$ (b), $\mathcal{N}=2$ (c) and $\mathcal{N}=4$ (d). Only eigenvalues with zero quasimomentum are displayed. 
As a next step, we examine the expression for the anomalous dimension of paired eigenstates with vanishing quasimomentum, Eq. (6.106), in SYM theory with $\mathcal{N}$ supercharges in the $\overline{\mathrm{DR}}$ scheme

$$
\Delta \gamma_{N=3}^{ \pm}(\lambda)=\lambda 4+\lambda^{2}\left[\frac{91}{12}-4 \mathcal{N}\right]
$$

Starting from two loops it depends linearly on the number of supercharges $\mathcal{N}$ and satisfies (5.64). It is easy to see that the two loop correction to $\Delta \gamma_{I}^{ \pm}(\lambda)$ is positive for $\mathcal{N}=1$ and negative for $\mathcal{N}=2$ and $\mathcal{N}=4$. As can be seen from Fig. 10 the same is true for higher values of conformal spin $N$.

The two-loop correction to (6.108) receives contributions from both two- and three-particle kernels, Eq. (5.46), and the question arises whether each of these contributions preserves integrability separately. To this end, one multiplies three-particle kernels in (5.46) by some factor $\xi$ and examines the $\xi$-dependence of the resulting expressions for the anomalous dimension. In this way, one finds

$$
\begin{aligned}
& \Delta \gamma_{\mathrm{I}}^{+}(\lambda)=\lambda 4+\lambda^{2}\left[\frac{5}{72} \xi+\frac{541}{72}-4 \mathcal{N}\right], \\
& \Delta \gamma_{\mathrm{I}}^{-}(\lambda)=\lambda 4+\lambda^{2}\left[\frac{1}{18} \xi+\frac{271}{36}-4 \mathcal{N}\right] .
\end{aligned}
$$

For $\xi=1$ one recovers (6.108) while for $\xi \neq 1$ the degeneracy is lifted. Thus, the two-loop integrability in SYM theories only arises in the sum of two- and three-particle contributions. ${ }^{10}$

Let us present the exact two-loop eigenspectrum for a few lowest anomalous dimensions with $\theta_{\boldsymbol{q}}=0$ in SYM theories with $\mathcal{N}$ supercharges. For odd conformal spin $N$ all eigenvalues with zero quasimomentum are paired and we denote the corresponding two-loop anomalous dimension matrix as $\Delta \gamma_{N}^{ \pm}$. For even $N$ there is an additional unpaired eigenstate with $q=0$ whose energy we denote as $\Delta \gamma_{N}$. Then one finds

$$
\begin{aligned}
& \Delta \gamma_{N=4}(\lambda)=\lambda \frac{19}{5}+\lambda^{2}\left[\frac{15581}{2250}-\frac{19}{5} \mathcal{N}\right] \\
& \Delta \gamma_{N=5}^{ \pm}(\lambda)=\lambda \frac{61}{12}+\lambda^{2}\left[\frac{55123}{5760}-\frac{61}{12} \mathcal{N}\right] \\
& \Delta \gamma_{N=6}^{ \pm}(\lambda)=\lambda \frac{401}{60}+\lambda^{2}\left[\frac{600697}{48000}-\frac{401}{60} \mathcal{N}\right] \\
& \Delta \gamma_{N=6}(\lambda)=\lambda \frac{341}{70}+\lambda^{2}\left[\frac{55402939}{6174000}-\frac{341}{70} \mathcal{N}\right]
\end{aligned}
$$

Notice the change of the sign of two-loop correction to the anomalous dimensions as one increases the amount of supersymmetry present in the model. While for QCD and $\mathcal{N}=1 \mathrm{SYM}$, the eigenvalues are strictly positive, the trend starts to change for $\mathcal{N}=2 \mathrm{SYM}$, where the lowspin anomalous dimensions are negative and decrease and then for higher $N$ the behavior starts to change and the spectrum recovers to positive values at higher conformal spins. For $\mathcal{N}=4$ SYM, the spectrum is entirely negative. This change in the tendency can be traced back to

\footnotetext{
${ }^{10}$ One may also wonder whether integrability would persist if one merely iterated the twist-two two-loop kernel $\mathbb{H}_{L=2}$ in all pairwise subchannels. A straightforward analysis demonstrates that the answer to this question is negative and the degeneracy of eigenvalues with zero quasimomentum is lifted in this case.
} 
the linear in $\mathcal{N}$ term in the right-hand side of (6.110) which in its turn comes from (5.64). It provides a large negative contribution to the anomalous dimensions for $\mathcal{N}=2$ and $\mathcal{N}=4$, which competes with the positive one from other terms.

\subsubsection{Large-spin limit}

One can see from Figs. 9 and 10 that the anomalous dimensions of the three-particle operators occupy a band

$$
\gamma_{\min }(\lambda) \leq \gamma_{\boldsymbol{q}}(\lambda) \leq \gamma_{\max }(\lambda)
$$

Let us demonstrate that both boundaries scale as $\sim \ln N$ at large conformal spins $N \gg 1$ with certain computable overall coefficients.

It is well known that the asymptotics of the anomalous dimensions at large conformal spins is governed by the terms in the evolution kernel which are singular for $v_{j} \rightarrow u_{j}$. This correspond to the situation when the wavelength of exchanged gluons is large. The behavior of the two-loop evolution kernels for $v_{j} \rightarrow u_{j}$ was scrutinized in Sects. 5.4.2 and 6.1.5. We have found in section 5.1 that the three-particle irreducible part of the three-particle kernel is not singular in this limit and, therefore, the leading asymptotic behavior of the anomalous dimensions for $N \gg 1$ emerges entirely from the two-particle irreducible kernels (5.55). According to (5.56), the all-loop evolution kernel $\mathbb{H}(\lambda)$ is given for $v_{j} \rightarrow u_{j}$ by a remarkably simple expression

$$
\mathbb{H}(\lambda)=\frac{1}{2} \Gamma_{\text {cusp }}(\lambda) \mathbb{H}^{(0)}+\ldots
$$

where $\mathbb{H}^{(0)}$ is the one-loop evolution kernel and ellipses denote terms subleading for $v_{j} \rightarrow u_{j}$. We would like to stress that this relation captures the leading $\sim \ln N$-terms in the anomalous dimension and neglects subleading $\sim N^{0}$ corrections.

The fact that $\mathbb{H}(\lambda)$ reduces to the one-loop evolution kernel accompanied by a multiplicative factor of $\Gamma_{\text {cusp }}(\lambda)$ implies that the all-loop dilatation operator is integrable in the large-spin limit both in QCD and SYM theories. The underlying integrable structure is the one corresponding to the classical Heisenberg magnet 44] with the Hamiltonian which can be cast into the form

$$
\mathbb{H}(\lambda)=\Gamma_{\text {cusp }}(\lambda) \ln q_{3, \mathrm{cl}}^{(0)}+O\left(N^{0}\right)
$$

with $q_{3, \mathrm{cl}}^{(0)}$ being the classical counterpart of the quantum integral of motion $q_{3}^{(0)}$. This leads to the following asymptotic behavior of the anomalous dimension

$$
\gamma_{\boldsymbol{q}}(\lambda)=\Gamma_{\mathrm{cusp}}(\lambda) \ln q+O\left(N^{0}\right)
$$

For $N \gg 1$, the conserved charge scales in the lower part of the spectrum as $q \sim \mathcal{O}\left(N^{2}\right)$ and in the upper one as $q \sim \mathcal{O}\left(N^{3}\right)$ so that

$$
\gamma_{\min }(\lambda)=2 \Gamma_{\text {cusp }}(\lambda) \ln N, \quad \gamma_{\max }(\lambda)=3 \Gamma_{\text {cusp }}(\lambda) \ln N
$$

These relations are in agreement with the results of Ref. 44. So far we studied the dilatation operator for nonlocal light-cone operator of length $L=3$. It worth mentioning that the relation (6.112) holds true for arbitrary $L$. 


\subsubsection{Large $\beta_{0}$-limit}

As follows from (6.106) and (6.107), the corrections to the two-loop anomalous dimensions coming from the terms involving the $\beta$-function preserve integrability. This may appear counterintuitive if one lands on the assumption that existence of integrability is intertwined with conformal symmetry. However, our findings demonstrate that the two phenomena are not related to one another.

We argued in Sect. 5.4.2 that one can enhance the contribution of conformal symmetry breaking terms $\sim \beta_{0}$ by going over to the formal limit $\beta_{0} \rightarrow \infty$ with $\lambda \beta_{0}=$ fixed. In this limit, the all-loop dilatation kernel is given by one-loop Feynman diagrams with the gluon propagator dressed by one-loop vacuum polarization bubble insertions. The resulting expression for the dilatation operator, Eq. (5.62), is proportional to the one-loop dilatation operator in which the "bare" conformal spin of quark/gaugino fields $j=1$ is replaced by its renormalized value $j=1+\beta_{0} g^{2} /\left(32 \pi^{2}\right)$.

The same effect can be understood within the $\varepsilon$-expansion as follows [56]. According to (4.66), for $D=4-2 \varepsilon$, the beta-function receives an additional contribution $\sim \varepsilon$. Therefore, there exists a value of $D=D_{\text {c }}$ for which the $D_{\mathrm{c}}$-dimensional $\beta$-function vanishes and the theory becomes conformal for this space-time dimension. It follows from (4.6) that this occurs for

$$
\varepsilon_{\mathrm{c}}=\frac{\beta\left(g^{2}\right)}{2}
$$

Since the canonical dimensions of fields depends on $D$, it will propagate into the value of the conformal spin, Eq. (6.13). For quarks/gauginos, one gets

$$
j=\frac{D_{\mathrm{c}}}{4}=1-\frac{\beta\left(g^{2}\right)}{4} \simeq 1+\frac{\beta_{0} g^{2}}{32 \pi^{2}}
$$

where in the last step we used the perturbative expansion (2.7). This precisely matches our finding in Eq. (5.62) for the renormalized conformal spin. Thus, the evolution kernel in the large $-\beta_{0}$ limit coincides with the evolution kernel in the conformal invariant field theory living in the "critical" $D_{\mathrm{c}}$-dimensions. ${ }^{11}$

We conclude that in the large- $\beta_{0}$ limit, the all-loop dilatation operator $\mathbb{H}(\lambda)$ is given by the one-loop dilatation operator with the shifted conformal spin (6.117). We recall that the latter operator coincides with the Hamiltonian of the Heisenberg $S L(2 ; \mathbb{R})$ spin chain. From the point of view of integrable models, renormalization of the conformal spin corresponds to deformation of unitary irreducible spin $j=1$ representation of the $S L(2) \sim S O(2,1)$ group to yet another irreducible but not unitary representation with spin $j=1+\beta_{0} g^{2} /\left(32 \pi^{2}\right)$. One can easily construct the "all loop-order" integrals of motion $q_{2}(\lambda)$ and $q_{3}(\lambda)$ for the corresponding system by merely substituting $j$ in Eqs. (6.66) and (6.71) by its renormalized value (6.117)

$$
q_{n}(\lambda)=\left.q_{n}^{(0)}\right|_{j=1+\frac{\beta_{0} g^{2}}{32 \pi^{2}}}=\mathrm{e}^{\lambda \mathbb{Z}} q_{n}^{(0)} \mathrm{e}^{-\lambda \mathbb{Z}} \quad(n=2,3)
$$

where the notation was introduced for the operator

$$
\mathbb{Z}=\frac{\beta_{0}}{4 c_{\mathrm{R}}} \frac{d}{d j} .
$$

\footnotetext{
${ }^{11}$ We are grateful to A. Manashov for proposing this interpretation.
} 
Expanding (6.118) in powers of $\lambda$ one reproduces (6.93).

The large $\beta_{0}$-limit provides a nontrivial example of all-loop dilatation operator and allows one to trace the deformation of integrable structures to higher loops. As in the previous section, the above analysis can be immediately generalized to the nonlocal light-cone operators of arbitrary length $L$.

\section{$7 \quad$ Discussion and conclusions}

In the present work we have derived the two-loop dilatation operator in the noncompact $S L(2)$ sector of QCD and SYM theories with $\mathcal{N}=1,2,4$ supercharges. We have chosen the three-quark and three-gaugino maximal-helicity operators, respectively, as a case of study. The choice was driven by the closure of the sector under two-loop renormalization and the simplicity of calculations involved. Supersymmetry allows one to extend our findings to the entire supermultiplet embodying operators with different particle content.

It is known that the dilatation operator in the aforementioned gauge theories is integrable to one-loop order in the sector under consideration. The major motivation for the current study was to verify whether integrability survives to two loops. To this end we had to use a guiding principle which will definitely point to its existence. We have used a criterion of double degeneracy of eigenvalues of the dilatation operator with zero quasimomentum. The eigenvalues with nonzero quasimomentum are always degenerate due to invariance of the dilatation operator under discrete symmetries - cyclic and parity permutations. We found that in QCD the degeneracy is lifted while in SYM theories it persists to two loop order for arbitrary number of supercharges $\mathcal{N}$. In the latter case, the spectrum of the two-loop dilatation operator depends on $\mathcal{N}$. We demonstrated that for the light-cone operator of arbitrary length $L=2,3, \ldots$ the $\mathcal{N}$-dependence has a remarkably simple form, Eq. (5.64). This implies that the dilatation operators in the $S L(2)$ sector of all SYM theories share the same integrability properties.

Making use of the degeneracy to two loops, we constructed the conserved charges $q_{2}(\lambda)$ and $q_{3}(\lambda)$ which commute with the two-loop Hamiltonian and among themselves. The first term in the perturbative expansion of these charges, $q_{2}^{(0)}$ and $q_{3}^{(0)}$, coincide with the integrals of motion of the $S L(2)$ Heisenberg magnet of length $L=3$ and spin $j=1$ [3, 4, 15. As such, they are given by conformal invariant polynomials in two-particle $S L(2)$ Casimirs $L_{k n}^{2}$ (with $k \neq n=1,2,3$ ), Eq. (6.18), and can be obtained from the auxiliary transfer matrix in a standard manner [21]. The interpretation of higher order corrections to $q_{2}(\lambda)$ and $q_{3}(\lambda)$ is an extremely nontrivial task both due to breaking of conformal symmetry in two loops and due to the fact that the $S L(2)$ spin magnet is noncompact. Namely, the two-particle Casimirs $L_{k n}^{2}$ are given by infinite-dimensional matrices in the conventional oscillatory basis and their powers are independent on each other. This should be contrasted with the compact $S U(2)$ spin-1/2 magnet in which case the spin operators are given by Pauli matrices and all powers of two-particle Casimirs $L_{k n}^{2}$ are expressed in terms of unity operators and its first power. As we noticed in Sect. 6.2.2, another possibility to identify integrable structures to two loops could be to perform a unitary transformation of the dilatation operator $\mathbb{H}(\lambda)$ and conserved charges $q_{n}(\lambda)$ with the operator $1+\lambda \mathbb{Z}$. It removes

higher order corrections from the conserved charges and yields the Hamiltonian $\widetilde{\mathbb{H}}=\mathbb{H}^{(0)}+\lambda \widetilde{\mathbb{H}}^{(1)}$ which commutes with $q_{n}^{(0)}$ (with $n=2,3$ )

$$
\left[q_{n}^{(0)}, \widetilde{\mathbb{H}}(\lambda)\right]=0+\mathcal{O}\left(\lambda^{2}\right) .
$$


Since the one-loop Hamiltonian is a function of the conserved charges $\mathbb{H}^{(0)}=\mathbb{H}^{(0)}\left(q_{2}^{(0)}, q_{3}^{(0)}\right)$, we conclude that the leading and the next-to-leading Hamiltonians are mutually commuting

$$
\left[\mathbb{H}^{(0)}, \widetilde{\mathbb{H}}^{(1)}\right]=0
$$

Thus, $\widetilde{\mathbb{H}}^{(1)}$ is a "higher" Hamiltonian of the $S L(2)$ Heisenberg spin chain. In principle, it can be constructed from $\mathbb{H}^{(0)}$ by applying the boost operator [57, 58] $B=\sum_{k} k \mathbb{H}_{k, k+1}^{(0)}$, that is $\left[B, \ldots\left[B, \mathbb{H}^{(0)}\right]\right]$, and forming their linear combination with suitably adjusted coefficients. In order to meet the parity requirement, only even powers of $B$ may appear in the expansion.

We demonstrated that the integrability is not tied to the survival of the conformal symmetry at two-loop order. On the contrary, in QCD case, the beta-function term in the dilatation operator preserves the pairing of eigenvalues contrary to the conformal invariant part of the Hamiltonian. This phenomenon carries on to SYM models where the conformal invariant part is integrable as well.

We found that the nonplanar diagrams provide vanishing contribution to the dilatation operator for $L=3$-particle operators in SYM models. For $L>3$, they induce next-to-nearest neighbor interactions at leading order, which are however suppressed by $1 / N_{c}^{2}$ with respect to the leading nearest-neighbor contributions. These $1 / N_{c}^{2}$-corrections explicitly break integrability but this phenomenon can be restored by going to the large- $N_{c}$ limit. Therefore, the large- $N_{c}$ limit is a necessary condition for integrability to survive. Note that to two-loop order there are nonplanar Feynman graphs (like in Figs. 2 (b) and 4 (b)). These diagrams were not considered presently since they are accompanied by a vanishing color structure for $L=3$ and also die out in the multi-color limit for $L>3$. For the QCD baryon operator the multi-color counting is not applicable since the corresponding operator exists only for $N_{c}=3$. Even if one sets by hand the nonplanar two-particle graph to zero, the pairing would not be restored due an intricate interplay between color factors of different Feynman diagrams in the fundamental and adjoint representations.

The two-loop dilatation operator has a rather complex structure in the momentum representation. It is however in this representation that higher-loop computation machinery is developed the best. It would be interesting to translate the obtained expressions into the coordinate, lightcone position representation and, eventually, write down the two-loop dilatation operator in terms of light-cone superfields as it was done to one-loop order in Ref. 24]. In this form, all symmetries of the dilatation operator are manifest and they may well hint on its possible structure to higher loops.

\section{Acknowledgements}

This work was supported by the U.S. National Science Foundation under grant no. PHY-0456520 (A.B. and D.M.). We are indebted to W. Vogelsang and F.-M. Dittes for providing us their notes on two-loop calculations which were indispensable at early stages of the project. We would also like to thank V. Braun, S. Derkachov and A. Manashov for useful discussions. A.B. and D.M. would like to thank Laboratoire de Physique Théorique (Orsay) for hospitality extended to them during their visit where a part of this work has been done. 


\section{A Forward limit}

In this Appendix we describe the limiting procedure which allows one to obtain the three-particle kernel in the forward limit $u_{1}+u_{2}+u_{3}=v_{1}+v_{2}+v_{3}=0$ from the expression (5.7) defined on the simplices (4.16) and (4.18).

As the first step, one extends the expression for the evolution kernel (5.7) to the entire domain $-\infty<u_{1}+u_{2}+u_{3}=v_{1}+v_{2}+v_{3}<\infty$ by making use of the support properties of defining Feynman diagrams. In the two-particle subchannels, the procedure is well-known and it amounts to substituting 36 ]

$$
\theta\left(v_{j}-u_{j}\right) \quad \rightarrow \quad \Theta\left(u_{a}, v_{a}\right)
$$

with generalized step function defined in (4.14). The analogous procedure can be formulated for the three-particle irreducible diagram too. As we found in Sect. 5.1, the three-particle kernel (5.7) is given by the sum of various $\mathbb{F}_{i}$-functions which are accompanied by the following step-function structures

$$
\begin{aligned}
& \vartheta_{123}^{(1)} \equiv \theta\left(u_{1}\right) \theta\left(u_{3}-v_{2}-v_{3}\right) \theta\left(1-u_{1}-u_{3}\right) \\
& \vartheta_{123}^{(2)} \equiv \theta\left(u_{1}\right) \theta\left(v_{1}-u_{1}\right) \theta\left(u_{3}-v_{3}\right) \theta\left(v_{2}+v_{3}-u_{3}\right) \\
& \vartheta_{123}^{(4)} \equiv \theta\left(u_{1}-v_{1}\right) \theta\left(u_{3}-v_{3}\right) \theta\left(1-u_{1}-u_{3}\right)
\end{aligned}
$$

The remaining two structures $\vartheta_{123}^{(i)}$ for $i=5,6$ are found from the above by permutation of arguments as in Eq. (5.8). It turns out that the generalized step-function structure can be restored through the following substitutions

$$
\begin{aligned}
& \vartheta_{123}^{(1)} \rightarrow \Theta_{123}^{(1)}=\Theta\left(u_{1}, v_{1}\right)\left[\Theta\left(u_{2}, 1-u_{1}-v_{3}\right)-\Theta\left(1-v_{1}-u_{3}, v_{2}\right)\right] \\
& \vartheta_{123}^{(2)} \rightarrow \Theta_{123}^{(2)}=\Theta\left(u_{1}, v_{1}\right) \Theta\left(1-v_{1}-u_{3}, v_{2}\right) \\
& \vartheta_{123}^{(4)} \rightarrow \Theta_{123}^{(4)}=\Theta\left(u_{2}, 1-v_{1}-u_{3}\right) \Theta\left(1-v_{1}-u_{3}, v_{2}\right)
\end{aligned}
$$

Having defined a generalized three-particle kernel in this way, the reduction to the forward limit can be easily performed with the help of (6.98). In the following we assume without loss of generality that $v_{1} \equiv y_{1} / \tau$ and $v_{3} \equiv y_{3} / \tau$ are positive in (6.98). Consequently, in the forward case we find for the building block (A.1):

$$
\operatorname{LIM} \Theta\left(u_{a}, v_{a}\right)=\left\{\begin{array}{ll}
\theta\left(x_{a}\right) \theta\left(y_{a}-x_{a}\right), & a=1,3 \\
-\theta\left(-x_{1}-x_{3}\right) \theta\left(x_{1}+x_{3}-y_{1}-y_{3}\right), & a=2
\end{array} .\right.
$$

The forward limit of Eqs. (A.5), (A.6), and (A.7) can be easily calculated and, one finds

$$
\begin{aligned}
& \operatorname{LIM} \Theta_{123}^{(1)}=\theta\left(x_{1}\right) \theta\left(y_{1}-x_{1}\right)\left[\theta\left(x_{3}+y_{1}\right)-\theta\left(x_{1}+x_{3}\right)\right] \theta\left(y_{3}-x_{3}\right), \\
& \operatorname{LIM} \Theta_{123}^{(2)}=-\theta\left(x_{1}\right) \theta\left(y_{1}-x_{1}\right) \theta\left(x_{3}+y_{1}\right) \theta\left(y_{3}-x_{3}\right), \\
& \operatorname{LIM} \Theta_{123}^{(4)}=\theta\left(x_{3}\right) \theta\left(y_{1}-x_{1}\right) \theta\left(x_{1}\right) \theta\left(y_{3}-x_{3}\right) .
\end{aligned}
$$




\section{B Subtraction terms}

The two-particle kernel (5.28) receives contribution from the subtraction terms (5.29) involving the integral of three-particle irreducible kernel

$$
W\left(u_{1}, u_{2} \mid v_{1}, v_{2}\right)=\int_{0}^{1} d u_{3}^{\prime} \mathbb{V}_{123}^{(1)}\left(u_{1}, 1-u_{1}-u_{3}^{\prime}, u_{3}^{\prime} \mid v_{1}, v_{2}, v_{3}\right)
$$

The evaluation of the integral is straightforward and leads to

$$
\begin{aligned}
W\left(u_{1}, u_{2} \mid v_{1}, v_{2}\right) & =\theta\left(\bar{u}_{1}\right) \theta\left(u_{1}-v_{2}-v_{1}\right) s_{1}\left(u_{1}, u_{2} \mid v_{1}, v_{2}\right) \\
& +\theta\left(u_{2}\right) \theta\left(v_{2}-u_{2}\right) s_{2}\left(u_{1}, u_{2} \mid v_{1}, v_{2}\right)+\theta\left(u_{1}\right) \theta\left(v_{1}-u_{1}\right) s_{3}\left(u_{1}, u_{2} \mid v_{1}, v_{2}\right)
\end{aligned}
$$

where the notation was introduced for the functions

$$
\begin{aligned}
s_{1}\left(u_{1}, u_{2} \mid v_{1}, v_{2}\right) & =-\frac{2 \bar{u}_{1}}{v_{3}\left(v_{1}-u_{1}\right)}-L\left(u_{1}-\bar{v}_{3}, v_{3}, v_{1}-u_{1}\right) \\
& -L\left(u_{1}-\bar{v}_{3}, v_{3}, v_{2}\right)+L\left(u_{1}-v_{1}, \bar{v}_{1}, v_{3}\right)+L\left(u_{1}-v_{1}, \bar{v}_{1}, v_{2}\right) \\
s_{2}\left(u_{1}, u_{2} \mid v_{1}, v_{2}\right) & =\frac{1}{2 v_{2}}\left(4-\ln ^{2} \frac{v_{2}-u_{2}}{v_{2}}\right) \\
& -L\left(u_{1}-v_{1}, v_{2}, v_{3}\right)+L\left(u_{1}-v_{1}, \bar{v}_{1}, v_{3}\right)+L\left(u_{1}-v_{1}, \bar{v}_{1}, v_{2}\right) \\
& -2 f\left(u_{2}, v_{2}\right)\left[\operatorname{Li}_{2}\left(\frac{u_{2}}{v_{2}}\right)-\zeta(2)\right]+\frac{v_{2}+v_{1}}{v_{2} v_{1}}\left[\operatorname{Li}_{2}\left(\frac{u_{2}}{v_{2}+v_{1}}\right)-\operatorname{Li}_{2}\left(\frac{u_{2}}{v_{2}}\right)\right] \\
s_{3}\left(u_{1}, u_{2} \mid v_{1}, v_{2}\right) & =f\left(u_{1}, v_{1}\right)\left[\frac{1}{2} \ln \frac{u_{2}}{v_{2}}+\ln \frac{u_{2}}{v_{2}} \ln \frac{u_{1}}{v_{1}}+2 \operatorname{Li}_{2}\left(\frac{v_{1}-u_{1}}{u_{2}}\right)\right] \\
& +\frac{v_{2}+v_{1}}{v_{2} v_{1}}\left[-\ln \frac{u_{2}}{v_{2}} \ln \frac{v_{1}}{v_{2}+v_{1}}+\operatorname{Li}_{2}\left(\frac{v_{1}-u_{1}}{u_{2}}\right)+\operatorname{Li}_{2}\left(\frac{v_{2}}{v_{2}+v_{1}}\right)-\zeta(2)\right]
\end{aligned}
$$

with the $f-$ and $L$-functions defined in (5.14) and (5.32), respectively.

The subtraction term (5.29) provides a genuine contribution to the two-particle irreducible kernel $\mathbb{V}_{12}$, Eq. (5.28). Similar contribution also comes from the gluon kernel (5.25) which involves the $\mathbb{V}_{\mathrm{SV}}^{(1)}(u, v)$-term. It is instructive to examine the sum of three terms

$$
\Delta \mathbb{V}_{12}(\boldsymbol{u} \mid \boldsymbol{v})=W\left(u_{1}, u_{2} \mid v_{1}, v_{2}\right)+W\left(u_{2}, u_{1} \mid v_{2}, v_{1}\right)+\frac{1}{u_{1}+u_{2}} \mathbb{V}_{\mathrm{SV}}^{(1)}\left(\frac{u_{1}}{u_{1}+u_{2}}, \frac{v_{1}}{v_{1}+v_{2}}\right)
$$

Taking into account Eqs. (B.2)-(B.5) and (15.24) we find that various terms cancel against each other leading to

$$
\begin{aligned}
\Delta \mathbb{V}_{12}(\boldsymbol{u} \mid \boldsymbol{v})=\theta\left(\bar{u}_{2}\right) \theta\left(u_{2}-v_{1}-v_{2}\right) \Delta \mathcal{F}_{1}\left(u_{1}, u_{2} \mid v_{1}, v_{2}\right) & +\theta\left(u_{1}\right) \theta\left(v_{1}-u_{1}\right) \Delta \mathcal{F}_{2}\left(u_{1}, u_{2} \mid v_{1}, v_{2}\right) \\
& +\left\{\begin{array}{l}
u_{1} \leftrightarrow u_{2} \\
v_{1} \leftrightarrow v_{2}
\end{array}\right\}
\end{aligned}
$$


with $\left(v_{3}=1-v_{1}-v_{2}\right)$

$$
\begin{aligned}
\Delta \mathcal{F}_{1}\left(u_{1}, u_{2} \mid v_{1}, v_{2}\right) & =-\frac{u_{1} \bar{u}_{2}}{v_{3}\left(v_{2}-u_{2}\right)}-L\left(u_{3}-\bar{v}_{1}, v_{3}, v_{2}-u_{2}\right)-L\left(u_{3}-\bar{v}_{1}, v_{3}, v_{2}\right) \\
& +L\left(u_{2}-v_{2}, \bar{v}_{2}, v_{3}\right)+L\left(u_{2}-v_{2}, \bar{v}_{2}, v_{1}\right), \\
\Delta \mathcal{F}_{2}\left(u_{1}, u_{2} \mid v_{1}, v_{2}\right) & =-L\left(u_{2}-v_{2}, v_{1}, v_{3}\right)+L\left(u_{2}-v_{2}, \bar{v}_{2}, v_{3}\right)+L\left(u_{2}-v_{2}, \bar{v}_{2}, v_{1}\right) \\
& +\frac{1}{2 v_{1}}\left(4-\ln ^{2} \frac{v_{1}-u_{1}}{v_{1}}\right)-\frac{1}{2} f\left(u_{1}, v_{2}\right)\left[3 \ln \frac{u_{1}}{v_{1}}+\ln ^{2} \frac{u_{1}}{v_{1}}\right] .
\end{aligned}
$$

We observe that it does not involve dilogarithms.

\section{References}

[1] L.N. Lipatov, JETP Lett. 59 (1994) 596.

[2] L.D. Faddeev, G.P. Korchemsky, Phys. Lett. B 342 (1995) 311;

G.P. Korchemsky, Nucl. Phys. B 443 (1995) 255.

[3] V.M. Braun, S.E. Derkachov, A.N. Manashov, Phys. Rev. Lett. 81 (1998) 2020;

V.M. Braun, S.E. Derkachov, G.P. Korchemsky, A.N. Manashov, Nucl. Phys. B 553 (1999) 355.

[4] A.V. Belitsky, Phys. Lett. B 453 (1999) 59; Nucl. Phys. B 558 (1999) 259; Nucl. Phys. B $574(2000) 407$.

[5] S.E. Derkachov, G.P. Korchemsky, A.N. Manashov, Nucl. Phys. B 566 (2000) 203.

[6] A.V. Belitsky, V.M. Braun, A.S. Gorsky, G.P. Korchemsky, Int. J. Mod. Phys. A 19 (2004) 4715.

[7] J.M. Maldacena, Adv. Theor. Math. Phys. 2 (1998) 231;

S.S. Gubser, I.R. Klebanov, A.M. Polyakov, Phys. Lett. B 428 (1998) 105;

E. Witten, Adv. Theor. Math. Phys. 2 (1998) 253.

[8] A.M. Polyakov, Int. J. Mod. Phys. A 17 S1 (2002) 119.

[9] D. Berenstein, J.M. Maldacena, H. Nastase, J. High Ener. Phys. 0204 (2002) 013.

[10] S.S. Gubser, I.R. Klebanov, A.M. Polyakov, Nucl. Phys. B 636 (2002) 99.

[11] S. Frolov, A.A. Tseytlin, J. High Ener. Phys. 0206 (2002) 007;

A.A. Tseytlin, Spinning strings and AdS/CFT duality, hep-th/0311139; Semiclassical strings and AdS/CFT hep-th/0409296.

[12] G. Mandal, N.V. Suryanarayana, S.R. Wadia, Phys. Lett. B 543 (2002) 81.

[13] I. Bena, J. Polchinski, R. Roiban, Phys. Rev. D 69 (2004) 046002.

[14] A.M. Polyakov, Mod. Phys. Lett. A 19 (2004) 1649. 
[15] L.N. Lipatov, Evolution equations in QCD, in Perspectives in Hadronic Physics, eds. S. Boffi, C. Ciofi Degli Atti, M. Giannini, World Scientific (Singapore, 1998) p. 413;

J.A. Minahan, K. Zarembo, J. High Ener. Phys. 0303 (2003) 013;

N. Beisert, M. Staudacher, Nucl. Phys. B 670 (2003) 439.

[16] N. Beisert, Phys. Rept. 407 (2004) 1.

[17] J. Plefka, Spinning strings and integrable spin chains in the AdS/CFT correspondence, hep-th/0507136.

[18] B. Eden, A two-loop test for the factorised S-matrix of planar $N=4$, hep-th/0501234.

[19] A.V. Belitsky, G.P. Korchemsky, D. Müller, Phys. Rev. Lett. 94 (2005) 151603.

[20] M. Grabowski, P. Mathieu, Mod. Phys. Lett. A 9 (1994) 2197.

[21] L.A. Takhtajan, L.D. Faddeev, Russ. Math. Survey 34 (1979) 11;

V.E. Korepin, N.M. Bogoliubov, A.G. Izergin, Quantum inverse scattering method and correlation functions, (Cambridge Univ. Press, 1993);

L.D. Faddeev, Int. J. Mod. Phys. A 10 (1995) 1845; Les Houches Lectures (1995), How algebraic Bethe ansatz works for integrable models, hep-th/9605187.

[22] A.P. Bukhvostov, G.V. Frolov, E.A. Kuraev, L.N. Lipatov, Nucl. Phys. B 258 (1985) 601.

[23] I.I. Balitsky, V.M. Braun, Nucl. Phys. B 311 (1989) 145.

[24] A.V. Belitsky, S.E. Derkachov, G.P. Korchemsky, A.N. Manashov, Phys. Lett. B 594 (2004) 385; Nucl. Phys. B 708 (2005) 115.

[25] J. Ralston, D.E. Soper, Nucl. Phys. B 152 (1979) 109.

[26] S.J. Brodsky, G.P. Lepage, Phys. Rev. D 22 (1980) 2157.

[27] A. Bassetto, G. Nardelli, R. Soldati, Yang-Mills Theories in the Algebraic non Covariant Gauges, World Scientific (Singapore, 1991).

[28] S. Mandelstam, Nucl. Phys. B 213 (1983) 149.

[29] G. Leibbrandt, Rev. Mod. Phys. 59 (1987) 1067.

[30] G. Curci, W. Furmanski, R. Petronzio, Nucl. Phys. B 175 (1980) 27.

[31] W. Siegel, Phys. Lett. B 84 (1979) 193.

[32] A.V. Belitsky, A.V. Radyushkin, Phys. Rept. 418 (2005) 1.

[33] A.V. Belitsky, D. Müller, Phys. Lett. B 417 (1998) 129.

[34] A.V. Radyushkin, Theor. Math. Phys. 61 (1985) 1144.

[35] R.L. Jaffe, Nucl. Phys. B 229 (1983) 205. 
[36] D. Müller, D. Robaschik, B. Geyer, F.M. Dittes, J. Hořejši, Phys. Lett. B 209 (1988) 325; Fortsch. Phys. 42 (1994) 101.

[37] W. Vogelsang, Phys. Rev. D 57 (1998) 1886;

S. Kumano, M. Miyama, Phys. Rev. D 56 (1997) 2504;

A. Hayashigaki, Y. Kanazawa, Y. Koike, Phys. Rev. D 56 (1997) 7350.

[38] A.V. Belitsky, D. Müller, A. Freund, Phys. Lett. B 493 (2000) 341.

[39] M.H. Sarmadi, Phys. Lett. B 143 (1984) 471.

[40] G.A. Schuler, S. Sakakibara, J.G. Korner, Phys. Lett. B 194 (1987) 125.

[41] G.P. Korchemsky, Mod. Phys. Lett. A 4 (1989) 1257;

G.P. Korchemsky, G. Marchesini, Nucl. Phys. B 406 (1993) 225.

[42] G.P. Korchemsky, A.V. Radyushkin, Nucl. Phys. B 283 (1987) 342.

[43] A.M. Polyakov, Nucl. Phys. B 164 (1980) 171.

[44] A.V. Belitsky, A.S. Gorsky, G.P. Korchemsky, Nucl. Phys. B 667 (2003) 3;

A.S. Gorsky, I.I. Kogan, G.P. Korchemsky, J. High Ener. Phys. 0205 (2002) 05.

[45] S.V. Mikhailov, Phys. Rev. D 62 (2000) 034002.

[46] V.M. Braun, G.P. Korchemsky, D. Müller, Prog. Part. Nucl. Phys. 51 (2003) 311.

[47] A.V. Efremov, A.V. Radyushkin, Phys. Lett. B 94 (1980) 245.

[48] Y.M. Makeenko, Sov. J. Nucl. Phys. 33 (1981) 440.

[49] T. Ohrndorf, Nucl. Phys. B 198 (1982) 26.

[50] D. Müller, Phys. Rev. D 49 (1994) 2525;

A.V. Belitsky, D. Müller, Nucl. Phys. B 537 (1999) 397.

[51] A.V. Kotikov, L.N. Lipatov, Nucl. Phys. B 661 (2003) 19;

A.V. Kotikov, L.N. Lipatov, V.N. Velizhanin, Phys. Lett. B 557 (2003) 114.

[52] F.A. Dolan, H. Osborn, Conformal partial wave expansion for $N=4$ chiral four-point functions, hep-th/0412335.

[53] A. Blasi, V.E.R. Lemes, N. Maggiore, S.P. Sorella, A. Tanzini, O.S. Ventura, L.C.Q. Vilar, J. High Ener. Phys. 0005 (2000) 039;

V.E.R. Lemes, M.S. Sarandy, S.P. Sorella, A. Tanzini, O.S. Ventura, J. High Ener. Phys. 0101 (2001) 016; Nonrenormalization theorems for $N=2$ super Yang-Mills, hep-th/0012197; N. Maggiore, A. Tanzini, Nucl. Phys. B 613 (2001) 34.

[54] A.V. Belitsky, S.É. Derkachov, G.P. Korchemsky, A.N. Manashov, Nucl. Phys. B 722 (2005) 191.

[55] G.P. Korchemsky, Nucl. Phys. B 462 (1996) 333. 
[56] A.N. Vasilev, The field theoretic renormalization group in critical behavior theory and stochastic dynamics, Chapman and Hall/CRC (Boca Raton, 2004).

[57] M.G. Tetelman, Sov. Phys. JETP 55 (1982) 306.

[58] E.K. Sklyanin, Quantum Inverse Scattering Method. Selected Topics, in Quantum Group and Quantum Integrable Systems (Nankai Lectures in Mathematical Physics), ed. Mo-Lin Ge, World Scientific (Singapore, 1992) p. 63; hep-th/9211111 